\title{
ALGÈBRES DE HECKE QUASI-ORDINAIRES UNIVERSELLES
}

\author{
PAR DAVID MAUGER
}

RÉSUMÉ. - Soit $\mathbf{G}$ un groupe réductif connexe sur $\mathbb{Q}$ dont le groupe adjoint possède des séries discrètes. Dans cet article, nous construisons une interpolation $p$-adique de l'ensemble des espaces de formes automorphes topologiques pour lequel nous étudions les propriétés nécessaires à la construction de familles $p$-adiques de systèmes de valeurs propres pour les opérateurs de Hecke. Nous imposons une hypothèse de quasi-ordinarité en $p$ et travaillons avec un système de coefficients de plus haut poids régulier. En supposant la conjecture de Leopoldt vérifiée, dans le cas d'un groupe unitaire à trois variables associé à une extension CM d'un corps totalement réel $F$, nous obtenons ainsi des familles $p$-adiques à $1+3[F: \mathbb{Q}]$ variables.

(C) 2004 Elsevier SAS

ABSTRACT. - Let $\mathbf{G}$ be a connected reductive group over $\mathbb{Q}$ whose adjoint group admits discrete series representations. In this article, we construct a $p$-adic interpolation of the set of spaces of topological automorphic forms and we study the required properties to construct $p$-adic families of Hecke eigensystems. We impose a nearly-ordinarity assumption at $p$ and we work with a local system of regular weight. Assuming Leopoldt conjecture, in the case of a unitary group in three variables associated with a CM extension of a totally real field $F$, we thus get $p$-adic families in $1+3[F: \mathbb{Q}]$ variables.

(c) 2004 Elsevier SAS

\section{Introduction}

Soit $\mathbf{G}$ un groupe réductif connexe sur $\mathbb{Q}$. Nous développons la théorie de Hida d'interpolation $p$-adique des espaces de cohomologie intérieure de la variété modulaire associée à $\mathbf{G}$. Cette théorie a déjà été mise en œuvre avec succès par Hida $[18,19]$ pour $\mathbf{G}=\mathbf{G} \mathbf{L}(n)$ et TilouineUrban [34] pour $\mathbf{G}=\mathbf{G S p}(4)$.

Nous construisons une grosse algèbre de Hecke $p$-adique $\mathbf{h}(\rho)$ obtenue comme limite projective d'algèbre de Hecke commutative agissant sur la cohomologie des variétés $\mathcal{M}_{K}$ dont le niveau en $p$ croît. Plus particulièrement, nous étudions les propriétés du facteur direct $\mathbf{h}_{\mathrm{qo}}(\rho)$ de cette algèbre découpé par l'idempotent $p$-quasi-ordinaire. Ce dernier est associé à un $\mathbb{Q}_{p}$-sousgroupe parabolique dont on fixe une décomposition de Levi $\mathbf{P}=\mathbf{M} \ltimes \mathbf{U}$. La théorie de Hida consiste à établir les résultats suivants :

- l'indépendance du poids de $\mathbf{h}_{\mathrm{qo}}(\rho)$;

- le contrôle de la localisation de $\mathbf{h}_{\mathrm{qo}}(\rho)$ aux idéaux premiers arithmétiques de l'algèbre de Hida-Iwasawa $\Lambda$;

- la finitude et la projectivité de $\mathbf{h}_{\mathrm{qo}}(\rho)$ sur $\Lambda$.

L'algèbre de Hida-Iwasawa est construite comme algèbre complétée du groupe formé par des opérateurs diamants en $p$. L'indépendance du poids est établie sous la forme suivante : l'algèbre $\mathbf{h}_{\mathrm{qo}}(\rho)$ est inchangée si on tord $\rho$ par un caractère arithmétique de $\mathbf{M}$. Nous obtenons le contrôle 
(à une erreur finie près) et la finitude lorsque $\rho$ est une représentation de plus haut poids régulier et le groupe adjoint $\mathbf{G}^{\text {ad }}$ de $\mathbf{G}$ possède des séries discrètes. Si de plus, la cohomologie de bas degré de $\mathcal{M}_{K}$ est sans $p$-torsion, $\mathbf{h}_{\mathrm{qo}}(\rho)$ est projective sur $\Lambda$.

Par exemple, soit GU le groupe des similitudes unitaires en trois variables associé à une extension $C M E$ d'un corps totalement réel $F$, anisotrope en toute place réelle sauf une et quasidéployé en $p$. Pour un tel groupe, $\Lambda$ est une algèbre de séries formelles à $N=1+3[F: \mathbb{Q}]+\delta_{E, p}$ variables, où $\delta_{E, p}$ est le défaut de la conjecture de Leopoldt pour le corps $E$ en $p$. On déduit de la théorie de Hida l'existence de familles $p$-adiques à $N$ variables de systèmes de valeurs propres pour les opérateurs de Hecke, quasi-ordinaires, passant par un tel système associé à une représentation automorphe $p$-quasi-ordinaire intervenant en poids régulier.

La première section est consacrée à l'équivariance sous les opérateurs de Hecke de la suite spectrale de Hochschild-Serre et de la restriction des scalaires, que nous établissons dans une très grande généralité.

Dans la deuxième section, nous utilisons la théorie de Bruhat-Tits pour munir $\mathbf{G}$ d'une structure entière sur $\mathbb{Z}_{p}$ adaptée à l'étude des opérateurs de Hecke en $p$ menée dans la section suivante.

Les variétés modulaires $\mathcal{M}_{K}$ et leur compactification de Borel-Serre sont introduites dans la quatrième section, où nous comparons leur cohomologie à celle du groupe de niveau $K$.

La cinquième section porte sur le passage à la limite sur le niveau et sur le thèorème de contrôle abstrait, établi à l'aide de la suite spectrale de Hochschild-Serre. Celui-ci constitue l'outil principal de la dernière section, consacrée aux propriétés de l'algèbre de Hecke quasiordinaire universelle $\mathbf{h}_{\mathrm{qo}}(\rho)$.

Il sera évident au lecteur combien notre travail est redevable aux travaux de Hida. Cette étude fut réalisée dans le cadre d'une thèse dirigée par Jacques Tilouine, que je remercie pour ses encouragements constants, et améliorée pendant un séjour postdoctoral à Jérusalem dans l'équipe d'Ehud de Shalit.

\section{Index des notations}

(1.1) $\mathbb{Z}[X], D, K, K^{\prime}, K^{\prime \prime}$, $\mathcal{H}\left(K, D, K^{\prime}\right), \mid, \mathcal{H}(K, D)$, anneau de Hecke.

(1.2) $K^{\prime}, \varphi_{\xi}$, sous-groupe distingué d'une paire.

(1.3) $\rho, \iota$.

(2) $\mathbf{G}, p, v_{p}$.

(2.1) $\mathbf{S}, \mathbf{Z}, \mathbf{N}, \Phi, W, V^{*}, \mathbf{R}_{\mathbb{Q}_{p}} \mathbf{G}, V,\langle\cdot, \cdot\rangle$, $H_{a}, s_{a}, A, \nu$, ord, $\mathrm{GA}(A), \mathrm{GL}(V)$, $\rho, \tilde{\mathbf{G}}, W_{a}, \alpha_{a, k}, \mathcal{I}$.

(2.2) $\Omega, \mathbf{G}_{\Omega}, \mathbf{U}_{a}, \mathbf{U}_{\alpha}, \Psi^{\text {réd }}, \mathbf{U}_{\Psi}$, $\mathbf{U}_{\Omega, a}, \mathbf{U}_{\Omega, \Psi}$.

(2.3) $\mathbf{M}, \mathbf{U}, \mathbf{P}, \mathbf{U}^{-}, \mathbf{P}^{-}, \Phi_{\mathbf{M}}, V_{\mathbf{M}}, A_{\mathbf{M}}$, $\nu_{\mathbf{M}}, \mathbf{R M}, L_{\mathbf{M}}$.
(2.4) partie bien placée, $\mathbf{G}_{\Omega}, \mathbf{M}_{\Omega}$, $\mathbf{P}_{\Omega}, \mathbf{P}_{\Omega}^{-}, \mathbf{U}_{\Omega}, \mathbf{U}_{\Omega}^{-}, \mathbf{R M}, F_{\mathbf{P}}, \Phi_{\mathbf{U}}$, $\Lambda_{\mathbf{M}}, \Lambda_{\mathbf{M}}^{+}, \mathbf{R M}\left(\mathbb{Q}_{p}\right)^{+}$, sous-groupe bien placé, $\mathcal{T}_{\mathrm{bp}}$.

(3) $\mathbf{M}_{\Omega}\left(\mathbb{Z}_{p}\right)^{*}, \mathbf{M}_{\Omega}\left(\mathbb{Z}_{p}\right)^{\prime}, \mathbf{P}_{\Omega}\left(\mathbb{Z}_{p}\right)^{*}$, $\mathbf{P}_{\Omega}\left(\mathbb{Z}_{p}\right)^{\prime}$.

(3.1) $\sigma, C, D_{1, p}, D_{0, p}$.

(3.2) $C_{U}$.

(4) G.

(4.1.1) $\mathbf{R G}, \mathbf{R}_{\mathbb{Q}} \mathbf{G}, \mathbf{R}_{\mathbb{R}} \mathbf{G}, \mathbf{R}_{\mathbb{Q}} \mathbf{G}(\mathbb{R})^{+}$, $\mathbf{R}_{\mathbb{R}} \mathbf{G}(\mathbb{R})^{+}, X, X^{\text {ad }}, \mathbf{G}^{\text {ad }}$.

(4.1.2) $K, \mathcal{M}_{K}, \Gamma_{H}$, $i_{g}, \alpha_{g, \gamma g k}$.

(4.2.1) $\mathfrak{P}, \mathfrak{P}_{1}, s(\mathbf{P}), \mathfrak{P}_{i}$, rang parabolique, $s(c)$. 
(4.2.2) $\mathbf{S}_{\mathbf{P}}, A_{\mathbf{P}}, \frac{a_{\mathbf{P}^{\prime}, \mathbf{P}}}{A_{\mathbf{P}}}, X(\mathbf{P})$,

(4.2.3) $\gamma_{\mathbf{P}}$. $e(\mathbf{P}), \bar{X}, \overline{e(\mathbf{P})}, \partial \bar{X}$.

(4.2.4) $\overline{\mathcal{M}}_{K}, \partial \overline{\mathcal{M}}_{K}$.

(4.2.5) $\partial_{c} \overline{\mathcal{M}}_{K}, \Gamma_{H}(\mathbf{P}), i_{\mathbf{P}, g}, \alpha_{\mathbf{P}, g, \gamma g k}$.

(4.3.1) assez petit, (TF), $\mathcal{T}$.

(4.3.2) $L, \mathcal{M}_{K}(L), i_{g}, \alpha_{g, \gamma g k}$.

(4.3.3) ${ }_{\xi} K, K_{\xi}$.

(4.3.4) $\operatorname{ind}^{\mathbf{G}\left(\mathbb{A}_{\mathrm{f}}\right)} L, \mathcal{F}(L)$.

(4.3.5) $\mathcal{M}_{K}^{\mathrm{ad}}, r_{\mathrm{RG}}$.

(4.3.7) $M(\chi), N(\chi)$.

(5) $p$.

(5.1.2) $\mathbb{A}_{\mathrm{f}}^{(S)}, \mathbb{A}_{\mathrm{f}}^{(p)}, K^{(p)}, D^{(p)}, \mathcal{H}^{(p)}$.

(5.1.3) $K_{1}, K_{0}, D_{1}, D_{0}, K_{*}, D_{*}$.

(5.2.1) $\mathcal{M}_{K_{*}}, \mathrm{H}^{\cdot}\left(\mathcal{M}_{K_{*}}, L\right)$.

(5.2.2) $\underline{\mathrm{H}}^{\cdot}\left(K_{*}, L\right)$.

(5.3) $\mathcal{O}, \mathcal{H}_{\mathcal{O}}^{(p)}$.

(5.3.2) $\overline{\mathcal{O}\left[\Lambda_{\mathrm{M}}^{+}\right]}, e_{\mathrm{qo}}$, idempotent quasi-ordinaire.

(5.4.1) $C_{p}, C^{(p)}, \mathfrak{p}_{C}, \mathfrak{m}_{C}$.

(5.4.3) $\mathcal{O}, \mathcal{K}, L(M)$.

(6) $\mathbf{G}$.

(6.1.1) $\rho, V(\mathcal{O}), L(\rho, \mathcal{K}), \mathcal{K}(\mathbf{G}), \mathbf{T}$, $\mathbf{B}^{-}, \chi_{\rho}$.

(6.1.2) $Y_{U}, L_{U}(\rho, \mathcal{O}), V(M)$, $L_{U}(\rho, M)$.
(6.1.4) $\delta, *$.

(6.1.5) $\omega_{\rho}$.

(6.1.6) RM, X* $(\mathbf{R M})^{-}, \omega$.

(6.2.1) caractère $\mathcal{O}$-arithmétique, $\varepsilon \chi, \mathfrak{p}_{\varepsilon \chi}$, $M(\varepsilon \chi), M[\varepsilon \chi]$.

(6.2.2) $\mathcal{T}_{\mathrm{bp}}(\varepsilon), L_{U}(\rho \otimes \varepsilon \chi, \cdot)$.

(6.2.3) $\mathcal{T}_{\mathrm{bp}}(r), V\left(p^{-r} \mathcal{O} / \mathcal{O}\right)(\chi), \mathrm{H}_{*}$, $Y_{U / U^{\prime}}, L_{U / U^{\prime}}(\rho \otimes \chi, \cdot)$, $\mathrm{h}_{*, U, \mathrm{qo}}\left(K_{1}, \rho \otimes \varepsilon \chi\right)$.

(6.3) $\mathcal{K}, \mathcal{K}_{0}$.

(6.3.1) $d$.

(6.3.2) $\mathbf{R}_{\mathbb{R}}^{\mathbb{Q}} \mathbf{G}$.

(6.3.3) $L(\rho, \mathcal{O}), \mathcal{O}\left(\mathbf{G}_{\Omega}\right), D_{p}, \delta, *, e_{\mathrm{qo}}$, $L(\rho, M), K$.

(6.3.4) P.

(6.3.5) GU, $F,\left(\mathrm{TF}_{\rho}\right)$.

(6.4) algèbre de Hecke $\mathbf{P}$-quasi-ordinaire universelle, $\mathbf{h}_{U, \mathrm{qo}}(\rho), \mathbb{V}_{U}(\rho)$.

(6.4.2) $\mathrm{h}_{!, U, \mathrm{qo}}\left(U^{\prime} K_{0}, \rho \otimes \varepsilon \chi\right)$.

(6.4.3) $C_{0}, \Lambda, H$, algèbre de Hida-Iwasawa.

(6.4.4) $\chi^{\vee}, w_{0}, \rho^{\vee}$.

(6.4.5) $\operatorname{rgp} \mathbf{P}, \delta_{\mathbf{R G}, p}, \overline{\mathbf{R G}(\mathbb{Z})}^{p}$.

(6.4.6) idéal arithmétique, caractère arithmétique, famille de systèmes de valeurs propres quasi-ordinaires.

\section{Opérateurs de Hecke abstraits}

Cette section réunit trois résultats concernant les anneaux de Hecke abstraits dont nous aurons besoin dans l'étude des opérateurs de Hecke en $p$. Le théorème 1.2.1, qui concerne l'équivariance de la suite spectrale de Hochschild-Serre, est essentiel pour démontrer les théorèmes de contrôle abstraits du paragraphe 5.4.3. Les propositions 1.3.1 et 1.4.1, plus classiques, portent respectivement sur l'équivariance des applications de restrictions (utilisée en 5.1.3 et 5.2.3) et les relations entre opérateurs de Hecke du type $K \xi K \mid K \nu K=K \xi \nu K$ (utilisées à plusieurs reprises dans la section 3).

Sauf précision contraire, les modules dont il est question dans cette section sont des modules à droite.

\subsection{Action sur la cohomologie des groupes}

Nous rappelons ici la définition des opérateurs de Hecke ( $c f$. [33, chapitre 3]) et de leur action sur la cohomologie des groupes ( $c f$. [22]).

Si $X$ est un ensemble, nous notons $\mathbb{Z}[X]$ le groupe abélien libre construit sur l'ensemble $X$. Lorsque $X$ est un monoïde, $\mathbb{Z}[X]$ est un anneau. 
Dans ce qui suit, nous fixons un monoïde $D$, dont $K, K^{\prime}$ et $K^{\prime \prime}$ sont des sous-groupes. Par extension des scalaires, les $K$-invariants d'un $D$-module $V$ sont donnés par

$$
\begin{gathered}
V^{K} \stackrel{\sim}{\longrightarrow} \operatorname{Hom}_{K}(\mathbb{Z}, V) \stackrel{\sim}{\longrightarrow} \operatorname{Hom}_{D}(\mathbb{Z}[K \backslash D], V) \\
v \longmapsto(K \xi \mapsto v \cdot \xi)
\end{gathered}
$$

$\operatorname{car} \mathbb{Z}[K \backslash D]=\mathbb{Z} \otimes_{\mathbb{Z}[K]} \mathbb{Z}[D]$ (ici $\mathbb{Z}$ est un $K$-module trivial et l'algèbre de monoïde $\mathbb{Z}[D]$ est un $D$-module (à droite) grâce à la représentation régulière à droite, et un $K$-module à gauche grâce à la restriction à $K$ de la représentation régulière à gauche).

En particulier, le groupe $\operatorname{Hom}_{D}\left(\mathbb{Z}\left[K^{\prime} \backslash D\right], \mathbb{Z}[K \backslash D]\right)$ s'identifie au groupe des $K^{\prime}$-invariants du $D$-module $\mathbb{Z}[K \backslash D]$, c'est-à-dire au groupe abélien libre, noté $\mathcal{H}\left(K, D, K^{\prime}\right)$, construit sur les classes doubles $K \xi K^{\prime} \subset D$ telles que $K \backslash K \xi K^{\prime}$ est fini. Les classes doubles vérifiant cette condition de finitude sont les opérateurs de Hecke (abstraits). Nous avons donc l'isomorphisme :

$$
\begin{aligned}
& \mathcal{H}\left(K, D, K^{\prime}\right) \stackrel{\sim}{\longrightarrow}(\mathbb{Z}[K \backslash D])^{K^{\prime}} \stackrel{\sim}{\longrightarrow} \operatorname{Hom}_{D}\left(\mathbb{Z}\left[K^{\prime} \backslash D\right], \mathbb{Z}[K \backslash D]\right) \\
& K \xi K^{\prime} \longmapsto\left(K^{\prime} \eta \longmapsto \sum_{K \theta \subset K \xi K^{\prime}} K \theta \eta\right) .
\end{aligned}
$$

Au vu de l'isomorphisme (1), la composition induit une loi bilinéaire

$$
\begin{aligned}
V^{K} \otimes_{\mathbb{Z}} \mathcal{H}\left(K, D, K^{\prime}\right) & \rightarrow V^{K^{\prime}} \\
v \otimes K \xi K^{\prime} & \mapsto v \mid K \xi K^{\prime}=\sum_{K \theta \subset K \xi K^{\prime}} v \cdot \theta .
\end{aligned}
$$

Pour $V=\mathbb{Z}\left[K^{\prime \prime} \backslash D\right]$, nous obtenons la loi de composition des opérateurs de Hecke :

$$
\begin{aligned}
\mathcal{H}\left(K^{\prime \prime}, D, K\right) \otimes_{\mathbb{Z}} \mathcal{H}\left(K, D, K^{\prime}\right) & \rightarrow \mathcal{H}\left(K^{\prime \prime}, D, K^{\prime}\right) \\
K^{\prime \prime} \nu K \otimes K \xi K^{\prime} & \mapsto K^{\prime \prime} \nu K \mid K \xi K^{\prime}:=\sum_{\substack{K^{\prime \prime} \eta \subset K^{\prime \prime} \nu K \\
K \theta \subset K \xi K^{\prime}}} K^{\prime \prime} \eta \theta .
\end{aligned}
$$

Elle est associative chaque fois que cela a un sens.

Notamment, $\mathcal{H}(K, D):=\mathcal{H}(K, D, K)$ est un anneau. C'est l'anneau de Hecke de la paire $K \subset D$. Cet anneau, isomorphe à l'anneau d'endomorphismes $\operatorname{End}_{D}(\mathbb{Z}[K \backslash D])$, agit (à droite) sur les $K$-invariants des $D$-modules. Transportée au membre de droite de l'isomorphisme (1), cette action est la composition.

Supposons, jusqu'à la fin de ce paragraphe, que $\mathbb{Z}[D]$ est un module plat à gauche sur $\mathbb{Z}[K]$. C'est le cas, par exemple, lorsque $D$ est un semi-groupe à droite car $\mathbb{Z}[D]$ est la somme directe, sur $K \xi \subset D$, des $K$-modules $\mathbb{Z}[K \xi] \simeq \mathbb{Z}[K]$. L'hypothèse de platitude implique qu'un module injectif sur $D$ est aussi injectif sur $K$, ce qui permet de dériver (1) et d'obtenir les isomorphismes :

$$
\mathrm{H}^{\cdot}(K, V) \simeq \operatorname{Ext}_{D}(\mathbb{Z}[K \backslash D], V)
$$


D'où une extension, qui conserve le degré, de la loi bilinéaire (3) :

$$
\begin{aligned}
\mathrm{H}^{\cdot}(K, V) \otimes_{\mathbb{Z}} \mathcal{H}\left(K, D, K^{\prime}\right) & \rightarrow \mathrm{H}^{\cdot}\left(K^{\prime}, V\right) \\
f \otimes K \xi K^{\prime} & \mapsto f \mid K \xi K^{\prime} .
\end{aligned}
$$

En particulier, l'anneau de Hecke $\mathcal{H}(K, D)$ agit sur la $K$-cohomologie des $D$-modules.

\section{2. Équivariance de la suite spectrale de Hochschild-Serre}

Maintenant, $K^{\prime}$ est un sous-groupe de $K$. Le résultat nouveau de cette section, énoncé dans le théorème 1.2.1, montre que la suite spectrale de Hochschild-Serre, pour un sous-groupe distingué de la paire $K \subset D$, est équivariante pour l'action des opérateurs de Hecke.

Pour définir la notion de sous-groupe distingué de la paire $K \subset D$, intéressons-nous au morphisme de $\mathcal{H}\left(K^{\prime}, D\right)$-modules à droite

$$
\begin{aligned}
\mathcal{H}\left(K^{\prime}, D\right) & \rightarrow \mathcal{H}\left(K, D, K^{\prime}\right) \\
K^{\prime} \xi K^{\prime} & \mapsto K 1 K^{\prime} \mid K^{\prime} \xi K^{\prime} .
\end{aligned}
$$

Pour tout $\xi \in D$, les fibres de l'application surjective

$$
\begin{aligned}
\varphi_{\xi}: K^{\prime} \backslash K^{\prime} \xi K^{\prime} & \rightarrow K \backslash K \xi K^{\prime} \\
K^{\prime} \eta & \mapsto K \eta
\end{aligned}
$$

ont toutes pour cardinal $\operatorname{deg}\left(\varphi_{\xi}\right)=\# K^{\prime} \backslash\left(K^{\prime} \xi K^{\prime} \cap K \xi\right)$. Si bien que, si $K^{\prime} \xi K^{\prime} \subset D$ est un opérateur de Hecke, $K 1 K^{\prime} \mid K^{\prime} \xi K^{\prime}=\operatorname{deg}\left(\varphi_{\xi}\right) K \xi K^{\prime}$. Nous disons que $K^{\prime}$ est un sous-groupe distingué de la paire $K \subset D$ lorsque

(i) $K^{\prime}$ est distingué dans $K$ et

(ii) pour chaque opérateur de Hecke $K \xi K^{\prime} \subset D$, l'application $\varphi_{\xi}$ est injective.

Les conséquences de la condition (i) sont les suivantes : le sous-anneau $\mathcal{H}\left(K^{\prime}, K\right) \subset$ $\mathcal{H}\left(K^{\prime}, D\right)$ est isomorphe à l'algèbre de groupe $\mathbb{Z}\left[K / K^{\prime}\right]$. Plus précisément, pour tout opérateur de Hecke $K^{\prime} \xi K^{\prime} \subset D$, on a

$$
\forall k, k^{\prime} \in K, \quad K^{\prime} k K^{\prime}\left|K^{\prime} \xi K^{\prime}\right| K^{\prime} k^{\prime} K^{\prime}=K^{\prime} k \xi k^{\prime} K^{\prime} .
$$

De plus, le noyau du morphisme (4) est l'idéal à droite $I_{K / K^{\prime}} \subset \mathcal{H}\left(K^{\prime}, D\right)$ engendré par $\left\{K^{\prime} k K^{\prime}-K^{\prime} 1 K^{\prime}\right\}_{k \in K} \operatorname{car} \operatorname{deg}\left(\varphi_{k \xi}\right)$ ne dépend pas de $k \in K$. La condition (ii) équivaut à la surjectivité du morphisme (4).

Quand $K^{\prime}$ est un sous-groupe distingué de la paire $K \subset D$, nous avons donc un isomorphisme

$$
\mathbb{Z} \otimes_{\mathbb{Z}\left[K / K^{\prime}\right]} \mathcal{H}\left(K^{\prime}, D\right) \simeq \mathcal{H}\left(K, D, K^{\prime}\right)
$$

Par extension des scalaires, les $K / K^{\prime}$-invariants d'un $\mathcal{H}\left(K^{\prime}, D\right)$-module $V$ sont donnés par :

$$
\begin{aligned}
V^{K / K^{\prime}} & \stackrel{\sim}{\rightarrow} \operatorname{Hom}_{\mathcal{H}\left(K^{\prime}, D\right)}\left(\mathcal{H}\left(K, D, K^{\prime}\right), V\right) \\
v & \mapsto\left(K \xi K^{\prime} \mapsto v \mid K^{\prime} \xi K^{\prime}\right) .
\end{aligned}
$$


En particulier, pour $V=\mathcal{H}\left(K, D, K^{\prime}\right)$, on obtient un isomorphisme

$$
\mathcal{H}(K, D) \simeq \operatorname{End}_{\mathcal{H}\left(K^{\prime}, D\right)}\left(\mathcal{H}\left(K, D, K^{\prime}\right)\right) .
$$

D'où une action de $\mathcal{H}(K, D)$ sur $V^{K / K^{\prime}}$. Le lemme suivant étend cette action à la $K / K^{\prime}$-cohomologie de $V$.

Pour dériver l'isomorphisme (6), un résultat de platitude est nécessaire :

LEMME 1.2.1. - Soient $K$ un sous-groupe d'un monö̈de $D$ et $K^{\prime}$ un sous-groupe distingué de la paire $K \subset D$.

Si $\mathbb{Z}[D]$ est un module plat à gauche sur $\mathbb{Z}[K]$, alors $\mathcal{H}\left(K^{\prime}, D\right)$ est un module plat à gauche sur $\mathbb{Z}\left[K / K^{\prime}\right]$ et pour tout $\mathcal{H}\left(K^{\prime}, D\right)$-module $V$, nous avons les isomorphismes

$$
\mathrm{H}^{\cdot}\left(K / K^{\prime}, V\right) \stackrel{\sim}{\rightarrow} \operatorname{Ext}_{\mathcal{H}\left(K^{\prime}, D\right)}\left(\mathcal{H}\left(K, D, K^{\prime}\right), V\right) .
$$

Démonstration. - Comme $\mathbb{Z}\left[K / K^{\prime}\right]$-module à gauche, $\mathcal{H}\left(K^{\prime}, D\right)$ est la somme directe, sur les opérateurs de Hecke $K \xi K^{\prime} \subset D$, des modules $\mathbb{Z}\left[K^{\prime} \backslash K \xi K^{\prime} / K^{\prime}\right]$.

La formule du degré de $\varphi_{\xi}$ montre que la projection naturelle est une bijection $K^{\prime} \backslash K \xi \simeq K^{\prime} \backslash K \xi K^{\prime} / K^{\prime}$. Or $\mathbb{Z}\left[K^{\prime} \backslash K \xi\right] \simeq \mathbb{Z}\left[K / K^{\prime}\right] \otimes_{\mathbb{Z}[K]} \mathbb{Z}[K \xi]$ est plat sur $\mathbb{Z}\left[K / K^{\prime}\right]$ car $\mathbb{Z}[K \xi]$ est un facteur direct du $\mathbb{Z}[K]$-module à gauche $\mathbb{Z}[D]$.

D'où la platitude de $\mathcal{H}\left(K^{\prime}, D\right)$ sur $\mathbb{Z}\left[K / K^{\prime}\right]$, ce qui permet, comme à la fin du paragraphe 1.1, de dériver l'isomorphisme (6).

ThÉORÈme 1.2.1. - Soient $K$ un sous-groupe d'un monoüde $D$ tel que $\mathbb{Z}[D]$ est un module plat à gauche sur $\mathbb{Z}[K]$ et $K^{\prime \prime} \subset K^{\prime}$ deux sous-groupes distingués de la paire $K \subset D$.

Pour tout $\mathcal{H}\left(K^{\prime \prime}, D\right)$-module à droite $V$, la suite spectrale de Hochschild-Serre

$$
E_{2}^{i, j}=\mathrm{H}^{i}\left(K / K^{\prime}, \mathrm{H}^{j}\left(K^{\prime} / K^{\prime \prime}, V\right)\right) \quad \Rightarrow \quad \mathrm{H}^{i+j}\left(K / K^{\prime \prime}, V\right)
$$

est Hecke-équivariante (i.e. $\mathcal{H}(K, D)$-équivariante).

Si $K^{\prime \prime}$ est trivial, nous obtenons :

COROllaire 1.2.1. - Soient $K$ un sous-groupe d'un monoüde $D$ tel que $\mathbb{Z}[D]$ est un module plat à gauche sur $\mathbb{Z}[K]$ et $K^{\prime}$ un sous-groupe distingué de la paire $K \subset D$.

Pour tout D-module à droite $V$, la suite spectrale de Hochschild-Serre

$$
E_{2}^{i, j}=\mathrm{H}^{i}\left(K / K^{\prime}, \mathrm{H}^{j}\left(K^{\prime}, V\right)\right) \quad \Rightarrow \quad \mathrm{H}^{i+j}(K, V)
$$

est Hecke-équivariante (i.e. $\mathcal{H}(K, D)$-équivariante).

Preuve du théorème 1.2.1. - L'isomorphisme (5) appliqué trois fois (à $K / K^{\prime}, K^{\prime} / K^{\prime \prime}$ puis $\left.K / K^{\prime \prime}\right)$ montre que la composition des opérateurs de Hecke définit un isomorphisme

$$
\mathcal{H}\left(K, D, K^{\prime}\right) \otimes_{\mathcal{H}\left(K^{\prime}, D\right)} \mathcal{H}\left(K^{\prime}, D, K^{\prime \prime}\right) \simeq \mathcal{H}\left(K, D, K^{\prime \prime}\right) .
$$

D'où, en tenant compte de (6), l'isomorphisme $\mathcal{H}(K, D)$-équivariant entre

$$
\begin{gathered}
\left(V^{K^{\prime} / K^{\prime \prime}}\right)^{K / K^{\prime}} \simeq \operatorname{Hom}_{\mathcal{H}\left(K^{\prime}, D\right)}\left(\mathcal{H}\left(K, D, K^{\prime}\right), \operatorname{Hom}_{\mathcal{H}\left(K^{\prime \prime}, D\right)}\left(\mathcal{H}\left(K^{\prime}, D, K^{\prime \prime}\right), V\right)\right) \\
\text { et } \quad V^{K / K^{\prime \prime}} \simeq \operatorname{Hom}_{\mathcal{H}\left(K^{\prime \prime}, D\right)}\left(\mathcal{H}\left(K, D, K^{\prime \prime}\right), V\right)
\end{gathered}
$$


Pour dériver cet isomorphisme et obtenir, grâce aux isomorphismes du lemme 1.2.1, la suite spectrale de $\mathcal{H}(K, D)$-modules annoncée, il suffit de vérifier la propriété d'acyclicité suivante : si $J$ est un $\mathcal{H}\left(K^{\prime \prime}, D\right)$-module injectif, alors $\mathrm{H}^{i}\left(K / K^{\prime}, J^{K^{\prime} / K^{\prime \prime}}\right)=0$ pour $i \geqslant 1$.

Or, par platitude à gauche de $\mathcal{H}\left(K^{\prime \prime}, D\right)$ sur $\mathbb{Z}\left[K / K^{\prime \prime}\right], J$ est injectif sur $K / K^{\prime \prime}$ et il est facile de vérifier que $J^{K^{\prime} / K^{\prime \prime}}$ est injectif sur $K / K^{\prime}$.

\section{3. Équivariance de l'application de restriction}

Nous nous intéressons ici à l'équivariance des applications de restriction en cohomologie des groupes. Ce qui nous assure que les opérateurs de Hecke agissant sur la cohomologie passent à la limite quand le niveau augmente ( $c f .5 .1 .3$ et 5.2.3).

Maintenant, $K^{\prime}$ est un sous-groupe de $K$ et $D^{\prime}$ est un sous-monoïde de $D$ contenant $K^{\prime}$. Et nous faisons l'hypothèse suivante : pour tout opérateur de Hecke $K^{\prime} \xi K^{\prime} \subset D^{\prime}, K \xi K^{\prime}=K \xi K$.

Cette hypothèse signifie exactement que le morphisme (4), restreint au sous-anneau $\mathcal{H}\left(K^{\prime}, D^{\prime}\right)$, se factorise sous la forme

$$
\begin{aligned}
\mathcal{H}\left(K^{\prime}, D^{\prime}\right) \stackrel{\rho}{\rightarrow} \mathcal{H}(K, D) \stackrel{\iota}{\hookrightarrow} \mathcal{H}\left(K, D, K^{\prime}\right) \\
K^{\prime} \xi K^{\prime} \mapsto \operatorname{deg}\left(\varphi_{\xi}\right) K \xi K
\end{aligned}
$$

où le morphisme

$$
\begin{aligned}
\iota: \mathcal{H}(K, D) & \hookrightarrow \mathcal{H}\left(K, D, K^{\prime}\right) \\
K \xi K & \mapsto K \xi K \mid K 1 K^{\prime}=\sum_{K \nu K^{\prime} \subset K \xi K} K \nu K^{\prime}
\end{aligned}
$$

identifie $\mathcal{H}(K, D)$ aux $K / K^{\prime}$-invariants de $\mathcal{H}\left(K, D, K^{\prime}\right)$, comme le prévoyait (2). Caractérisé par l'égalité

$$
\rho\left(K^{\prime} \xi K^{\prime}\right)\left|K 1 K^{\prime}=K 1 K^{\prime}\right| K^{\prime} \xi K^{\prime}
$$

l'application $\rho$ est un morphisme d'anneaux : si $K^{\prime} \xi K^{\prime}$ et $K^{\prime} \eta K^{\prime} \subset D^{\prime}$ sont des opérateurs de Hecke, on a

$$
\begin{aligned}
\rho\left(K^{\prime} \xi K^{\prime}\right)\left|\rho\left(K^{\prime} \eta K^{\prime}\right)\right| K 1 K^{\prime} & =\rho\left(K^{\prime} \xi K^{\prime}\right)\left|K 1 K^{\prime}\right| K^{\prime} \eta K^{\prime} \\
& =K 1 K^{\prime}\left|K^{\prime} \xi K^{\prime}\right| K^{\prime} \eta K^{\prime} \\
& =\rho\left(K^{\prime} \xi K^{\prime} \mid K^{\prime} \eta K^{\prime}\right) \mid K 1 K^{\prime} .
\end{aligned}
$$

Puisque l'opérateur de Hecke $K 1 K^{\prime}$ induit l'application de restriction en cohomologie des groupes, nous obtenons :

Proposition 1.3.1. - Soient $K$ un sous-groupe d'un monö̈de $D$ tel que $\mathbb{Z}[D]$ est un module plat à gauche sur $\mathbb{Z}[K], K^{\prime}$ est un sous-groupe de $K$ et $D^{\prime}$ un sous-monoüde de $D$ contenant $K^{\prime}$ tel que, pour tout opérateur de Hecke $K^{\prime} \xi K^{\prime} \subset D^{\prime}, K \xi K^{\prime}=K \xi K$.

Alors pour tout $D$-module à droite $V$, les morphismes de restriction

$$
H^{\cdot}(K, V) \rightarrow H^{\cdot}\left(K^{\prime}, V\right)
$$

sont Hecke-équivariants, i.e. compatibles avec le morphisme d'anneaux $\rho$.

Cette proposition est une légère généralisation du théorème 2.7.6 de Miyake [24], qui supposait que, pour tout opérateur $K^{\prime} \xi K^{\prime}\left(\xi \in D^{\prime}\right)$, l'application $\varphi_{\xi}$ est injective. 


\subsection{Anneaux de Hecke et algèbres de monoïde}

Nous donnons ici un critère numérique pour qu'un anneau de Hecke soit isomorphe à l'algèbre d'un monoïde. Ce critère est la formalisation de l'argument utilisé par Hida [19, section 2] pour déterminer la structure de certaines algèbres de Hecke locales pour un sous-groupe de type $\Gamma_{0}$ de $\mathbf{G}=\mathbf{S L}_{n}$. Nous utilisons ce critère dans la section 3 pour généraliser le résultat de Hida aux groupes réductifs connexes $\mathbf{G}$.

Proposition 1.4.1. - Soient $K, K^{\prime}$ et $K^{\prime \prime}$ des sous-groupes d'un monoüde D. Quels que soient les opérateurs de Hecke $K^{\prime \prime} \nu K \subset D$ et $K \xi K^{\prime} \subset D$ on a l'inégalité

$$
\#\left(K^{\prime \prime} \backslash K^{\prime \prime} \nu K\right) \#\left(K \backslash K \xi K^{\prime}\right) \geqslant \#\left(K^{\prime \prime} \backslash K^{\prime \prime} \nu \xi K^{\prime}\right) .
$$

Et l'égalité est équivalente à $K^{\prime \prime} \nu K \mid K \xi K^{\prime}=K^{\prime \prime} \nu \xi K^{\prime}$.

Démonstration. - L'inégalité vient de

$$
K^{\prime \prime} \nu \xi K^{\prime} \subset K^{\prime \prime} \nu K \xi K^{\prime}=\bigcup_{\substack{K^{\prime \prime} \eta \subset K^{\prime \prime} \nu K \\ K \theta \subset K \xi K^{\prime}}} K^{\prime \prime} \eta \theta .
$$

Et l'égalité est équivalente à $K^{\prime \prime} \nu \xi K^{\prime}=K^{\prime \prime} \nu K \xi K^{\prime}$ et le fait que l'union à droite est disjointe. Ce qui signifie exactement $K^{\prime \prime} \nu K \mid K \xi K^{\prime}=K^{\prime \prime} \nu \xi K^{\prime}$ d'après la définition de la composition des opérateurs de Hecke.

Pour $K=K^{\prime}=K^{\prime \prime}$, on obtient :

COROllaire 1.4.1. - Soient $K$ un sous-groupe d'un monoüde $D$ et $D^{\prime}$ un sous-monö̈de de $D$ sur lequel la fonction $\xi \mapsto \#(K \backslash K \xi K)$ est à valeurs finies et multiplicative.

Alors $K D^{\prime} K$ est un sous-monoüde de $D$, dont la loi passe au quotient double $K \backslash K D^{\prime} K / K$ et on a l'isomorphisme d'anneaux :

$$
\begin{aligned}
& \mathbb{Z}\left[K \backslash K D^{\prime} K / K\right] \stackrel{\sim}{\rightarrow} \mathcal{H}\left(K, K D^{\prime} K\right) \\
& K \xi K \mapsto K \xi K .
\end{aligned}
$$

\section{Théorie de Bruhat-Tits}

Nous rappelons ici brièvement ce dont nous aurons besoin dans la section suivante de la théorie de Bruhat-Tits [10] pour construire la donnée dont nous déduirons, au paragraphe 3, l'anneau de Hecke parabolique.

Ici, G est un groupe réductif connexe sur $\mathbb{Q}_{p}$. Nous notons $v_{p}$ la valuation $p$-adique sur $\mathbb{Q}_{p}$.

\subsection{Un appartement}

On fixe $\mathbf{S}$ un tore $\mathbb{Q}_{p}$-déployé maximal de $\mathbf{G}$ dont on note $\mathbf{Z}$ (resp. $\mathbf{N}$ ) le centralisateur (resp. normalisateur) dans $\mathbf{G}$. Soient $\Phi \subset \mathrm{X}^{*}(\mathbf{S})$ le système de racines (relatives à $\mathbb{Q}_{p}$ ) de $\mathbf{G}$ par rapport à $\mathbf{S}, W:=\mathbf{N} / \mathbf{Z}=\mathbf{N}\left(\mathbb{Q}_{p}\right) / \mathbf{Z}\left(\mathbb{Q}_{p}\right)$ le groupe de Weyl correspondant et $V^{*} \subset \mathbb{R} \otimes_{\mathbb{Z}} \mathbf{X}^{*}(\mathbf{S})$ le sous-espace-vectoriel réel engendré par $\Phi$. Soit $\mathbf{R}_{\mathbb{Q}_{p}} \mathbf{G}$ le radical $\mathbb{Q}_{p}$-déployé (i.e. le plus grand tore $\mathbb{Q}_{p}$-déployé central) de $\mathbf{G}$. La dualité (de groupes abéliens libres) entre caractères et cocaractères de $\mathbf{S}$ induit une dualité (d'espaces vectoriels réels) entre $V^{*}$ et

$$
V=\mathbb{R} \otimes_{\mathbb{Z}}\left(\mathrm{X}_{*}(\mathbf{S}) / \mathrm{X}_{*}\left(\mathbf{R}_{\mathbb{Q}_{p}} \mathbf{G}\right)\right)
$$


Lorsqu'on parlera d'orthogonalité, ce sera pour cette dernière dualité. Ces dualités sont notées $\langle\cdot, \cdot\rangle$.

Pour toute racine $a \in \Phi$, on note $H_{a}:=\operatorname{ker}\langle a, \cdot\rangle \subset V$ l'ensemble des points fixes de la réflexion $s_{a} \in W$ de $V$ associée à $a$.

L'appartement $A$ associé à $\mathbf{S}$ est un espace affine sous $V$, muni d'une action de $\mathbf{N}\left(\mathbb{Q}_{p}\right)$ par automorphismes affines :

- Le sous-groupe $\mathbf{Z}\left(\mathbb{Q}_{p}\right)$ agit par translation sur $A$. Plus précisément, l'action de $t \in \mathbf{Z}\left(\mathbb{Q}_{p}\right)$ sur $A$ est la translation par le vecteur image $\nu(t)$ de $-\operatorname{ord}(t)$ dans $V$ où

$$
\mathbf{Z}\left(\mathbb{Q}_{p}\right) \stackrel{\text { ord }}{\longrightarrow} \mathbb{R} \otimes_{\mathbb{Z}} \mathrm{X}_{*}(\mathbf{S})
$$

est le morphisme de groupes déterminé par

$$
\left\langle a_{\mid \mathbf{S}}, \operatorname{ord}(t)\right\rangle=v_{p}(a(t))
$$

pour tout $t \in \mathbf{Z}\left(\mathbb{Q}_{p}\right)$ et tout caractère $\mathbb{Q}_{p}$-rationnel $a$ de $\mathbf{Z}$.

- L'action de $\mathbf{N}\left(\mathbb{Q}_{p}\right)$ sur $A$, encore notée $\nu$, est une extension de celle du groupe de Weyl $W$ sur $V$ par celle de $\mathbf{Z}\left(\mathbb{Q}_{p}\right)$ par translation sur $A$. Autrement dit, on a un morphisme de suites exactes courtes de groupes :

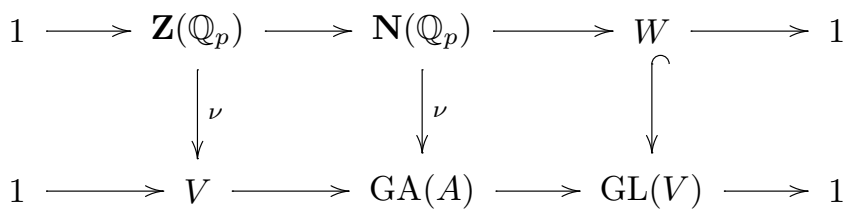

dans lequel $\mathrm{GA}(A)$ (resp. GL( $V)$ ) est le groupe affine (resp. linéaire) de $A$ (resp. $V$ ) et $V$ est identifié au groupe des translations de $A$.

En fait, l'existence et l'unicité (à translation près) d'une telle représentation affine de $\mathbf{N}\left(\mathbb{Q}_{p}\right)$ sur $A$ sont la conséquence de la nullité des groupes de cohomologie $\mathrm{H}^{i}(W, V), i=1,2$.

Soit $\rho: \tilde{\mathbf{G}} \rightarrow \mathbf{G}$ le revêtement universel du groupe dérivé de $\mathbf{G}$. Le sous-groupe

$$
W_{\mathrm{a}}:=\nu\left(\mathbf{N}\left(\mathbb{Q}_{p}\right) \cap \rho\left(\tilde{\mathbf{G}}\left(\mathbb{Q}_{p}\right)\right)\right) \subset \mathrm{GA}(A)
$$

est un groupe de Weyl affine au sens de Bruhat-Tits [10, I.1.3]. En particulier $W_{\mathrm{a}}$, muni de la topologie discrète, opère proprement sur $A$ et est engendré par des réflexions par rapport à des hyperplans affines. Si on munit $V$ d'un produit scalaire invariant par $W$, ces réflexions sont orthogonales pour la structure euclidienne associée sur $A$. De plus $W_{\mathrm{a}}$ est le produit semidirect du réseau $\nu\left(\mathbf{Z}\left(\mathbb{Q}_{p}\right) \cap \rho\left(\tilde{\mathbf{G}}\left(\mathbb{Q}_{p}\right)\right)\right)$ de $V$ par $W$. Par conséquent, d'après [7, VI, §1, n ${ }^{\circ}$, rem. 3], les applications linéaires associées aux réflexions de $W_{\mathrm{a}}$ sont les réflexions $s_{a}, a \in \Phi$. Un hyperplan, ensemble des points fixes d'une réflexion de $A$ appartenant à $W_{\mathrm{a}}$, est appelé mur de $A$. Selon ce qui précède, sa direction est l'un des hyperplans $H_{a} \subset V, a \in \Phi$. Une racine affine de $A$ est un demi-espace affine fermé limité par un mur. Si un point $x$ de $A$ est donné, les racines affines sont de la forme

$$
\alpha_{a, k}:=\{x+v \mid v \in V,\langle a, v\rangle+k \geqslant 0\} \quad \text { où } a \in \Phi, k \in \mathbb{R} .
$$

Une telle racine affine $\alpha_{a, k}$ est dite associée à $a$ (cette notion ne dépend évidemment pas du point $x \in A$ ). Une facette de $A$ est une classe d'équivalence dans $A$ pour la relation d'appartenance 
aux mêmes racines affines. Une alcôve de $A$ est une composante connexe du complémentaire dans $A$ de la réunion des murs. Son adhérence est un domaine fondamental pour l'action de $W_{\mathrm{a}}$ dans $A[7, \mathrm{~V}, \S 3$, th. 2]. Un point de $A$ est un point spécial si, pour tout mur de $A$, il existe un translaté, par un élément de $V \cap W_{\mathrm{a}}$, de ce mur contenant ce point.

L'immeuble de Bruhat-Tits $\mathcal{I}$ de $\mathbf{G}$ sur $\mathbb{Q}_{p}$ est un quotient du produit contracté

$$
\mathbf{G}\left(\mathbb{Q}_{p}\right) \times{ }^{\mathbf{N}\left(\mathbb{Q}_{p}\right)} A=\coprod_{\mathbf{G}\left(\mathbb{Q}_{p}\right) / \mathbf{N}\left(\mathbb{Q}_{p}\right)} g A
$$

par une relation d'équivalence compatible avec l'action de $\mathbf{G}\left(\mathbb{Q}_{p}\right)$ [10, I.7.4.2]. De plus, l'application canonique $A \rightarrow \mathcal{I}$ identifie $A$ à une partie de l'immeuble dont le stabilisateur est $\mathbf{N}\left(\mathbb{Q}_{p}\right)$. Les appartements (resp. murs, facettes, alcôves, points spéciaux) de $\mathcal{I}$ sont les transformés de $A$ (resp. des murs, facettes, alcôves, points spéciaux de $A$ ) par un élément de $\mathbf{G}\left(\mathbb{Q}_{p}\right)$. Le choix d'un produit scalaire invariant par $W$ sur $V$ permet $[10$, I.2.5.4] de munir l'immeuble $\mathcal{I}$ d'une distance invariante sous $\mathbf{G}\left(\mathbb{Q}_{p}\right)$ laquelle, restreinte à l'appartement $A$, est induite par la structure euclidienne provenant de $V$.

\subsection{Schémas en groupes sur $\mathbb{Z}_{p}$}

La théorie de Bruhat-Tits associe [10, II.5.1.30], à toute partie bornée non vide $\Omega$ d'un appartement de l'immeuble $\mathcal{I}$, un schéma en groupes $\mathbf{G}_{\Omega}$ affine, lisse sur $\mathbb{Z}_{p}$, à fibres connexes, de fibre générique $\mathbf{G}\left(\mathbf{G}_{\Omega}\right.$ ne dépend pas du choix d'un appartement contenant $\Omega$ ). Le groupe des points entiers $\mathbf{G}_{\Omega}\left(\mathbb{Z}_{p}\right)$, appelé fixateur connexe de $\Omega$, est un sous-groupe ouvert compact de $\mathbf{G}\left(\mathbb{Q}_{p}\right)$ qui fixe $\Omega$. Par définition [10, II.5.2.6 et 8], un $\mathbb{Q}_{p}$-sous-groupe parahorique (resp. un $\mathbb{Q}_{p}$-sous-groupe d'Iwahori), est le fixateur connexe d'une facette (resp. une alcôve) de $\mathcal{I}$.

La construction de $\mathbf{G}_{\Omega}$ se fait en plusieurs étapes. Selon un théorème de Steinberg [32, III.2], G est quasi-déployé sur une extension galoisienne finie et non ramifiée $F$ de $\mathbb{Q}_{p}$, d'anneau d'entiers $\mathcal{O}_{F}$ et de groupe de Galois $\Gamma$. L'immeuble $\mathcal{I}$ est identifié [10, II.5] à l'ensemble des points fixes de $\Gamma$ dans l'immeuble de $\mathbf{G}$ sur $F$. On construit d'abord un réseau $M_{\Omega}$ dans une représentation algébrique fidèle de $\mathbf{G}_{F}\left(c f\right.$. [10, II.2 à 4]) et ensuite on obtient $\mathbf{G}_{\Omega}$ par descente étale de $\mathcal{O}_{F}$ à $\mathbb{Z}_{p}$ à partir de la composante neutre de l'adhérence schématique de $\mathbf{G}_{F}$ dans le groupe linéaire $\mathbf{G L}\left(M_{\Omega}\right)$.

Supposons que $\Omega$ est une partie bornée non vide de l'appartement $A$ associé au tore $\mathbb{Q}_{p}$-déployé maximal S. Selon [10, II.1.2.6-7], l'adhérence schématique d'un sous-groupe fermé $\mathbf{H}$ de $\mathbf{G}$ dans $\mathbf{G}_{\Omega}$ est un sous- $\mathbb{Z}_{p}$-schéma en groupes fermé de $\mathbf{G}_{\Omega}$ et c'est l'unique sous- $\mathbb{Z}_{p^{-}}$schéma fermé plat de $\mathbf{G}_{\Omega}$ de fibre générique $\mathbf{H}$.

On note $\mathbf{U}_{a}$ le sous-groupe radiciel de $\mathbf{G}$ associé à la racine $a \in \Phi$ : c'est le plus grand sousgroupe fermé connexe de $\mathbf{G}$ normalisé par $\mathbf{S}$ et tel que les racines $a \in \Phi$ intervenant dans la représentation adjointe de $\mathbf{S}$ dans l'algèbre de Lie de $\mathbf{U}_{a}$ sont des multiples entiers positifs de $a$.

La théorie de Bruhat-Tits munit, grâce à un épinglage, le groupe $\mathbf{U}_{a}\left(\mathbb{Q}_{p}\right)$ d'une filtration décroissante et exhaustive $\left(\mathbf{U}_{\alpha}\right)_{\alpha}$ indexée par l'ensemble des racines affines $\alpha$ associées à $a$, ordonné par inclusion [10, I.6.2.6]. De plus, les sous-groupes $\mathbf{U}_{\alpha}$ forment un système fondamental de voisinages ouverts compacts de l'unité dans $\mathbf{U}_{a}\left(\mathbb{Q}_{p}\right)$. Et, selon [10, I.6.2.10(iii)], pour toute racine affine $\alpha$ et $n \in \mathbf{N}\left(\mathbb{Q}_{p}\right)$, on a ${ }^{n}\left(\mathbf{U}_{\alpha}\right)=\mathbf{U}_{n . \alpha}$, où $n . \alpha$ est l'image de la racine affine $\alpha$ par $n$. Ici et dans la suite, on note ${ }^{x} H:=x H x^{-1}$ et $H^{x}:=x^{-1} H x$ pour tout sous-groupe ou élément $H$ d'un groupe $G(x \in G)$.

En particulier, $\mathbf{Z}\left(\mathbb{Q}_{p}\right)$ normalise $\mathbf{U}_{a}$ et permute l'ensemble (indexé par les racines affines $\alpha$ associées à $a$ ) des sous-groupes $\mathbf{U}_{\alpha}$. 
Si $\Psi$ est une partie close de $\Phi$ (i.e. telle que pour tous $a, b \in \Psi$, si $a+b \in \Phi$ alors $a+b \in \Psi$ ) contenue dans un demi-espace ouvert de $\mathbb{R} \otimes_{\mathbb{Z}} \mathrm{X}^{*}(\mathbf{S})$, alors (cf. [6, prop. 3.11]), quel que soit l'ordre mis sur $\Psi^{\text {réd }}:=\{a \in \Psi \mid a / 2 \notin \Psi\}$ l'application schématique produit

$$
\prod_{a \in \Psi^{\text {réd }}} \mathbf{U}_{a} \rightarrow \mathbf{G}
$$

est un isomorphisme de schémas sur le sous-groupe fermé $\mathbf{U}_{\Psi}$ engendré par les $\mathbf{U}_{a}, a \in \Psi$.

Les faits suivants sont des conséquences de la construction de $\mathbf{G}_{\Omega}$ :

(i) l'adhérence schématique de $\mathbf{S}$ dans $\mathbf{G}_{\Omega}$ s'identifie au $\mathbb{Z}_{p}$-schéma canonique $\operatorname{Spec} \mathbb{Z}_{p}\left[\mathrm{X}^{*}(\mathbf{S})\right]$ de fibre générique $\mathbf{S}$ (cf. [10, II.3.8.3 ( $\left.\mathrm{S}^{\circ} 1\right)$ et 4.4 .18 (II)] dans le cas $\mathbb{Q}_{p}$-quasi-déployé, puis [10, II.5.1.9] dans le cas général);

(ii) pour toute racine $a \in \Phi$, l'adhérence schématique $\mathbf{U}_{\Omega, a}$ de $\mathbf{U}_{a}$ dans $\mathbf{G}_{\Omega}$ est l'unique $\mathbb{Z}_{p}$-schéma en groupes affine, lisse de fibre générique $\mathbf{U}_{a}$ tel que $\mathbf{U}_{\Omega, a}\left(\mathbb{Z}_{p}\right)=\mathbf{U}_{\alpha}$ où $\alpha$ est la plus petite racine affine associée à $a$ contenant $\Omega\left(c f\right.$. [10, II.3.8.3 $\left.\left(\mathrm{S}^{\circ} 1\right)\right]$ dans le cas $\mathbb{Q}_{p}$-quasi-déployé puis [10, II.5.2.2] dans le cas général);

(iii) l'application schématique produit

$$
\prod_{a \in \Psi^{\text {réd }}} \mathbf{U}_{\Omega, a} \rightarrow \mathbf{G}_{\Omega}
$$

prolonge (10) en un isomorphisme de schémas sur l'adhérence schématique $\mathbf{U}_{\Omega, \Psi}$ de $\mathbf{U}_{\Psi}$ dans $\mathbf{G}_{\Omega}\left(c f\right.$. [10, II.3.8.3 ( $\left.\left.\mathbf{S}^{\circ} 2\right)\right]$ dans le cas $\mathbb{Q}_{p}$-quasi-déployé puis [10, II.5.2.3] dans le cas général).

(iv) l'adhérence schématique d'un tore $\mathbf{T}$ de $\mathbf{Z}$ s'identifie, dans la terminologie de [10, II.4.4], au lissifié du $\mathbb{Z}_{p}$-schéma canonique de fibre générique $\mathbf{T}$. En particulier, le groupe de ses $\mathbb{Z}_{p}$-points est le sous-groupe compact maximal de $\mathbf{T}\left(\mathbb{Q}_{p}\right)$.

\subsection{Immeuble d'un sous-groupe de Levi}

Nous fixons une décomposition de Levi $\mathbf{M} \ltimes \mathbf{U} \simeq \mathbf{P}$ d'un $\mathbb{Q}_{p}$-sous-groupe parabolique de G. Le sous-groupe de Levi $\mathbf{M}$ est le centralisateur de son radical $\mathbb{Q}_{p}$-déployé $\mathbf{R}_{\mathbb{Q}_{p}} \mathbf{M}$ dans $\mathbf{G}$. Nous notons $\mathbf{M} \ltimes \mathbf{U}^{-} \simeq \mathbf{P}^{-}$la décomposition de Levi du sous-groupe parabolique opposé à $\mathbf{P}$ contenant $\mathbf{M}$.

Ayant choisi un tore $\mathbb{Q}_{p}$-déployé maximal $\mathbf{S}$ de $\mathbf{M}$, nous reprenons les notations $\Phi, V, A$, $\nu$ de 2.1, que nous affectons de l'indice $\mathbf{M}$ pour désigner les mêmes objets relatifs au groupe réductif $M$.

LEMME 2.3.1. - Soit $\mathbf{R M}$ le radical de $\mathbf{M}$. Le sous-espace réel

$$
L_{\mathbf{M}}:=\mathbb{R} \otimes_{\mathbb{Z}}\left(\mathrm{X}_{*}\left(\mathbf{R}_{\mathbb{Q}_{p}} \mathbf{M}\right) / \mathrm{X}_{*}\left(\mathbf{R}_{\mathbb{Q}_{p}} \mathbf{G}\right)\right) \subset V
$$

est l'orthogonal de $\Phi_{\mathrm{M}}$ et on a le diagramme commutatif suivant :

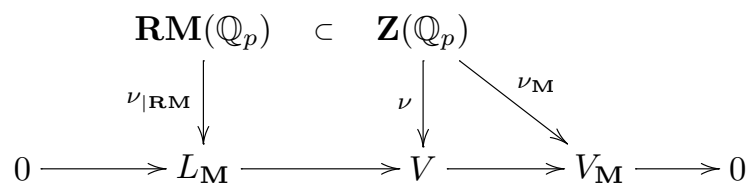

dont la ligne inférieure est une suite exacte courte. 
Démonstration. - Le tore $\mathbb{Q}_{p}$-déployé $\mathbf{R}_{\mathbb{Q}_{p}} \mathbf{M}$ est la composante neutre de $\bigcap_{a \in \Phi_{\mathbf{M}}}$ ker $a$. Ainsi les cocaractères de $\mathbf{R}_{\mathbb{Q}_{p}} \mathbf{M}$ sont exactement les cocaractères de $\mathbf{S}$ orthogonaux aux racines $a \in \Phi_{\mathbf{M}}$, et $L_{\mathbf{M}}$ s'identifie à l'orthogonal de $\Phi_{\mathbf{M}}$ dans $V$.

Par définition, $V_{\mathbf{M}}:=\mathbb{R} \otimes_{\mathbb{Z}}\left(\mathrm{X}_{*}(\mathbf{S}) / \mathrm{X}_{*}\left(\mathbf{R}_{\mathbb{Q}_{p}} \mathbf{M}\right)\right)$ car $\mathbf{R}_{\mathbb{Q}_{p}} \mathbf{M}$ est le radical $\mathbb{Q}_{p}$-déployé de $\mathbf{M}$, d'où l'exactitude de la ligne inférieure. La commutativité du triangle est évidente puisque les deux morphismes $\nu$ et $\nu_{\mathbf{M}}$ se déduisent de - ord.

Il reste à montrer que pour tout $t \in \mathbf{R M}\left(\mathbb{Q}_{p}\right)$ et tout $a \in \Phi_{\mathbf{M}},\langle a, \nu(t)\rangle=0$. On peut se restreindre aux $a$ appartenant à un système de racines simples de $\Phi_{\mathbf{M}}$.

D'après [15, XIV, th. 1.1], le groupe $\mathbf{Z}$ contient un tore maximal $\mathbf{T}$ défini sur $\mathbb{Q}_{p}$. Nécessairement, il contient $\mathbf{S}$ et c'est aussi un tore maximal de $\mathbf{G}$, contenu dans $\mathbf{M}$. Ainsi $\mathbf{S}$ et $\mathbf{R M}$ sont des sous-tores de $\mathbf{T}$. Selon la proposition [6, 6.8], $a$ se prolonge en une racine $\tilde{a}$ de $\mathbf{M}$ par rapport à $\mathbf{T}$. La nullité de $\langle a, \nu(t)\rangle=-\langle\tilde{a}, \operatorname{ord}(t)\rangle$ résulte du lemme suivant appliqué à $\tilde{a} \in \mathrm{X}^{*}(\mathbf{T})$.

LEMME 2.3.2. - Soit $\mathbf{T}$ un tore maximal du groupe $\mathbf{Z}$.

Pour tout $t \in \mathbf{R M}\left(\mathbb{Q}_{p}\right)$ et tout $a \in \mathrm{X}^{*}(\mathbf{T})$ on $a\left\langle a_{\mid \mathbf{S}}, \operatorname{ord}(t)\right\rangle=v_{p}(a(t))$, où nous avons étendu la valuation $v_{p}$ à une clôture algébrique de $\mathbb{Q}_{p}$.

En particulier, si a appartient à l'ensemble $\Phi_{\mathbf{M}}(\mathbf{T})$ des racines de $\mathbf{M}$ par rapport à $\mathbf{T}$, on a $a(t)=1$ et $\left\langle a_{\mid \mathbf{S}}, \operatorname{ord}(t)\right\rangle=0$.

Dans ce lemme, on ne suppose pas que $\mathbf{T}$ est défini sur $\mathbb{Q}_{p}$.

Démonstration.-Puisque $\mathbf{T}$ est un tore maximal de $\mathbf{Z}$, le morphisme de restriction $\mathrm{X}^{*}(\mathbf{Z}) \rightarrow \mathrm{X}^{*}(\mathbf{T})$ est injectif à conoyau fini. Il existe donc un multiple $N a\left(N \in \mathbb{N}^{*}\right)$ de $a$ qui s'étend en un caractère $a^{\prime}$ de $\mathbf{Z}$.

Soit $\tilde{F}$ une extension galoisienne finie de $\mathbb{Q}_{p}$ sur laquelle $a^{\prime}$ est défini. On note $\tilde{\Gamma}$ son groupe de Galois et $\tilde{d}$ son degré.

La formule (8) appliquée au caractère $\sum_{\sigma \in \tilde{\Gamma}}{ }^{\sigma} a^{\prime}$, nous donne :

$$
\left\langle\sum_{\sigma \in \tilde{\Gamma}} \sigma_{a}^{\prime} a_{\mid \mathbf{S}}, \operatorname{ord}(t)\right\rangle=v_{p}\left(\sum_{\sigma \in \tilde{\Gamma}} \sigma_{a}^{\prime}(t)\right) .
$$

Or $\mathbf{S}$ est un tore $\mathbb{Q}_{p}$-déployé et $a_{\mid \mathbf{S}}^{\prime}=N a_{\mid \mathbf{S}}$, donc le membre de gauche vaut $\tilde{d} N\left\langle a_{\mid \mathbf{S}}, \operatorname{ord}(t)\right\rangle$. Quant à celui de droite, il vaut $\tilde{d} N v_{p}(a(t)) \operatorname{car} t \in \mathbf{R M}\left(\mathbb{Q}_{p}\right)$ et $a_{\mid \mathbf{R M}}^{\prime}=N a_{\mid \mathbf{R M}}$.

La dernière assertion vient du fait que les racines de $\mathbf{M}$ par rapport à $\mathbf{T}$ sont triviales sur le radical de $\mathbf{M}$.

La dernière partie de la preuve du lemme 2.3.1 redémontre le fait que le radical d'un groupe réductif agit trivialement sur son immeuble de Bruhat-Tits.

Selon [10, I.7.6.3-4], l'appartement $A_{\mathbf{M}}$ de l'immeuble de $\mathbf{M}$ associé à $\mathbf{S}$ s'identifie à $A / L_{\mathbf{M}}$, muni de l'action quotient de $\mathbf{N}\left(\mathbb{Q}_{p}\right) \cap \mathbf{M}\left(\mathbb{Q}_{p}\right)$. Et d'après [10, II.4.2.15], l'immeuble d'un groupe s'identifie à celui de son groupe adjoint, donc aussi à celui de son groupe dérivé.

\subsection{Propriété de contraction}

Nous dirons qu'une partie bornée non vide $\Omega$ de $\mathcal{I}$ est bien placée par rapport au couple $(\mathbf{P}, \mathbf{M})$ si elle est incluse dans l'appartement associé à un tore $\mathbb{Q}_{p}$-déployé maximal $\mathbf{S}$ de $\mathbf{M}$. Nous fixons une telle partie $\Omega$ et un tel tore $\mathbf{S}$. Soit $\mathbf{G}_{\Omega}$ (resp. $\mathbf{M}_{\Omega}$ ) le schéma affine lisse sur $\mathbb{Z}_{p}$, à fibres connexes et de fibre générique $\mathbf{G}$ (resp. $\mathbf{M}$ ) associé ( $c f .2 .2)$ à $\Omega \subset A$ (resp. au projeté de $\Omega$ sur $\left.A_{\mathbf{M}}\right)$. On note $\mathbf{P}_{\Omega}\left(\right.$ resp. $\mathbf{P}_{\Omega}^{-}, \mathbf{U}_{\Omega}, \mathbf{U}_{\Omega}^{-}$) l'adhérence schématique de $\mathbf{P}$ (resp. $\mathbf{U}, \mathbf{U}^{-}$) dans 
$\mathbf{G}_{\Omega}$. On note encore $\mathbf{R M}$ l'adhérence schématique de $\mathbf{R M}$ dans $\mathbf{G}_{\Omega}$. Selon 2.2(iv), $\mathbf{R M}\left(\mathbb{Z}_{p}\right)$ est le sous-groupe compact maximal de $\mathbf{R M}\left(\mathbb{Q}_{p}\right)$.

PROPOSITION 2.4.1.-

(i) L'immersion fermée $\mathbf{M} \rightarrow \mathbf{G}$ se prolonge en un isomorphisme de $\mathbb{Z}_{p}$-schémas en groupes de $\mathbf{M}_{\Omega}$ sur le centralisateur dans $\mathbf{G}_{\Omega}$ du $\mathbb{Z}_{p}$-schéma canonique de fibre générique $\mathbf{R}_{\mathbb{Q}_{p}} \mathbf{M}$.

Ainsi $\mathbf{M}_{\Omega}$ s'identifie à l'adhérence schématique de $\mathbf{M}$ dans $\mathbf{G}_{\Omega}$.

(ii) $\mathbf{M}_{\Omega}$ normalise $\mathbf{U}_{\Omega}$ et la décomposition de Levi $\mathbf{M} \ltimes \mathbf{U} \simeq \mathbf{P}$ se prolonge en un isomorphisme

$$
\mathbf{M}_{\Omega} \ltimes \mathbf{U}_{\Omega} \stackrel{\sim}{\rightarrow} \mathbf{P}_{\Omega}
$$

(iii) Le morphisme produit

$$
\mathbf{U}_{\Omega}^{-} \times \mathbf{M}_{\Omega} \times \mathbf{U}_{\Omega} \rightarrow \mathbf{G}_{\Omega}
$$

est un isomorphisme sur un voisinage ouvert de la section unité de $\mathbf{G}_{\Omega}$.

Démonstration. - D'après 2.2(i), l'adhérence schématique du tore $\mathbf{R}_{\mathbb{Q}_{p}} \mathbf{M}$ dans $\mathbf{G}_{\Omega}$ est le tore $\mathbb{Z}_{p}$-déployé $\mathbf{S p e c} \mathbb{Z}_{p}\left[\mathrm{X}^{*}\left(\mathbf{R}_{\mathbb{Q}_{p}} \mathbf{M}\right)\right]$, son centralisateur, dans $\mathbf{G}_{\Omega}$, est un sous-schéma en groupes fermé, lisse sur $\mathbb{Z}_{p}$ et à fibres connexes [15, XIX, 2.2]. De plus, sa fibre générique est le centralisateur de $\mathbf{R}_{\mathbb{Q}_{p}} \mathbf{M}$ dans $\mathbf{G}$, c'est-à-dire $\mathbf{M}$. Ces propriétés caractérisent ( $c f .2 .2$ ) l'adhérence schématique de $\mathbf{M}$ dans $\mathbf{G}_{\Omega}$.

L'identification de $\mathbf{M}_{\Omega}$ avec l'adhérence schématique de $\mathbf{M}$ s'obtient grâce à la caractérisation [10, II.3.8.3] de $\mathbf{M}_{\Omega}$ dans le cas $\mathbb{Q}_{p}$-quasi-déployé, puis par descente étale dans le cas général. D'où (i).

Les deux assertions (ii) et (iii) sont la conséquence directe du théorème [10, II.2.2.3] appliqué au tore $\mathbb{Q}_{p}$-déployé $\mathbf{R}_{\mathbb{Q}_{p}} \mathbf{M}$.

Le lemme suivant énonce les propriétés de contraction que nous utiliserons pour décrire la structure des algèbres de Hecke paraboliques.

Les sous-groupes paraboliques $\mathbf{P}$ et $\mathbf{P}^{-}$, de l'appartement de l'immeuble de Tits de $\mathbf{G}$ associé à $\mathbf{S}$, correspondent respectivement aux facettes vectorielles $F_{\mathbf{P}}$ et $-F_{\mathbf{P}}$ de $V$, où

$$
F_{\mathbf{P}}:=\left\{v \in L_{M} \mid \forall a \in \Phi_{\mathbf{U}},\langle a, v\rangle>0\right\}
$$

et $\Phi_{\mathbf{U}}$ est l'ensemble des racines $a \in \Phi$ intervenant dans la représentation adjointe de $\mathbf{S}$ dans l'algèbre de Lie de $\mathbf{U}$.

L'image $\Lambda_{\mathbf{M}}:=\nu\left(\mathbf{R M}\left(\mathbb{Q}_{p}\right)\right)$ est un réseau de $L_{\mathbf{M}}$. Soit $\Lambda_{\mathbf{M}}^{+}$le monoïde commutatif libre intersection de $\Lambda_{\mathbf{M}}$ avec l'adhérence de $F_{\mathbf{P}}$ et $\mathbf{R M}\left(\mathbb{Q}_{p}\right)^{+}$son image réciproque par $\nu_{\mid \mathbf{R M}}\left(\mathbb{Q}_{p}\right)$. On a donc

$$
\mathbf{R M}\left(\mathbb{Q}_{p}\right)^{+}=\left\{t \in \mathbf{R M}\left(\mathbb{Q}_{p}\right) \mid \forall a \in \Phi_{\mathbf{U}},-v_{p}(a(t))=\langle a, \nu(t)\rangle \geqslant 0\right\} .
$$

Selon le lemme 2.3.2, si $\Phi_{\mathbf{U}}(\mathbf{T})$ est l'ensemble des racines de $\mathbf{U}$ par rapport à un tore maximal $\mathbf{T}$ (non nécessairement défini sur $\mathbb{Q}_{p}$ ) de $\mathbf{Z}$, on a aussi

$$
\mathbf{R M}\left(\mathbb{Q}_{p}\right)^{+}=\left\{t \in \mathbf{R M}\left(\mathbb{Q}_{p}\right) \mid \forall a \in \Phi_{\mathbf{U}}(\mathbf{T}), v_{p}(a(t)) \leqslant 0\right\}
$$

car selon $[6,6.8]$, toute racine $a \in\left(\Phi_{\mathbf{U}}\right)^{\text {réd }}$ se prolonge en une racine $a^{\prime} \in \Phi_{\mathbf{U}}(\mathbf{T})$.

LeMme 2.4.1. - Pour tout $t \in \mathbf{R M}\left(\mathbb{Q}_{p}\right)^{+}$, on a les relations 


$$
\begin{aligned}
\mathbf{M}_{\Omega}\left(\mathbb{Z}_{p}\right)^{t}= & \mathbf{M}_{\Omega}\left(\mathbb{Z}_{p}\right) \quad \mathbf{U}_{\Omega}\left(\mathbb{Z}_{p}\right)^{t} \subset \mathbf{U}_{\Omega}\left(\mathbb{Z}_{p}\right) \quad \mathbf{U}_{\Omega}^{-}\left(\mathbb{Z}_{p}\right)^{t} \supset \mathbf{U}_{\Omega}^{-}\left(\mathbb{Z}_{p}\right) \\
& \mathbf{P}_{\Omega}\left(\mathbb{Z}_{p}\right)^{t} \subset \mathbf{P}_{\Omega}\left(\mathbb{Z}_{p}\right) \quad \mathbf{P}_{\Omega}^{-}\left(\mathbb{Z}_{p}\right)^{t} \supset \mathbf{P}_{\Omega}^{-}\left(\mathbb{Z}_{p}\right) .
\end{aligned}
$$

De plus, les sous-groupes $\mathbf{U}_{\Omega}\left(\mathbb{Z}_{p}\right)^{t}$ (resp. ${ }^{t} \mathbf{U}_{\Omega}^{-}\left(\mathbb{Z}_{p}\right)$ ), $t \in \mathbf{R M}\left(\mathbb{Q}_{p}\right)^{+}$, forment un système fondamental de voisinages ouverts compacts de l'unité dans $\mathbf{U}\left(\mathbb{Q}_{p}\right)$ (resp. $\mathbf{U}^{-}\left(\mathbb{Q}_{p}\right)$ ).

Démonstration. - La relation faisant intervenir $\mathbf{M}$ est immédiate car RM est la composante neutre du centre de $\mathbf{M}$.

Pour obtenir celle concernant $\mathbf{U}$, il suffit, d'après 2.2(ii) et (iii) appliqué à $\Psi=\Phi_{\mathbf{U}}$, de montrer, pour tout $a \in\left(\Phi_{\mathbf{U}}\right)^{\text {réd }}$, l'inclusion $\left(\mathbf{U}_{\alpha}\right)^{t} \subset \mathbf{U}_{\alpha}$ où $\alpha$ est la plus petite racine associée à $a$, contenant $\Omega$. Or, selon 2.2, on a $\left(\mathbf{U}_{\alpha}\right)^{t}=\mathbf{U}_{\alpha-\nu(t)}$, donc il suffit d'avoir $\alpha+\nu(t) \subset \alpha$. Ce qui équivaut, puisque $\alpha$ est de la forme (9), à $\langle a, \nu(t)\rangle \geqslant 0$.

D'après la décomposition de Levi de la proposition 2.4.1, ces deux premières relations donnent celle pour $\mathbf{P}$.

En remplaçant $\mathbf{U}$ par $\mathbf{U}^{-}$, on obtient de même les relations concernant $\mathbf{U}^{-}$et $\mathbf{P}^{-}$.

Et la dernière assertion résulte de l'assertion analogue pour les sous-groupes $\mathbf{U}_{\alpha} \subset \mathbf{U}_{a}\left(\mathbb{Q}_{p}\right)$ (cf. 2.2).

Soit $K$ un sous-groupe distingué de $\mathbf{G}_{\Omega}\left(\mathbb{Z}_{p}\right)$. Il est bien placé par rapport à $(\mathbf{P}, \mathbf{M})$ s'il se décompose sous la forme

$$
K=\left(K \cap \mathbf{U}_{\Omega}^{-}\left(\mathbb{Z}_{p}\right)\right)\left(K \cap \mathbf{M}_{\Omega}\left(\mathbb{Z}_{p}\right)\right)\left(K \cap \mathbf{U}_{\Omega}\left(\mathbb{Z}_{p}\right)\right)
$$

et si pour tout $t \in \mathbf{R M}\left(\mathbb{Q}_{p}\right)^{+}$,

$$
\left(K \cap \mathbf{U}_{\Omega}^{-}\left(\mathbb{Z}_{p}\right)\right)^{t} \supset K \cap \mathbf{U}_{\Omega}^{-}\left(\mathbb{Z}_{p}\right)
$$

Il résulte de [30, I.2] que l'ensemble, noté $\mathcal{T}_{\mathrm{bp}}$, des sous-groupes ouverts, distingués dans $\mathbf{G}_{\Omega}\left(\mathbb{Z}_{p}\right)$ et bien placés par rapport à $(\mathbf{P}, \mathbf{M})$ forment un système fondamental de voisinages ouverts de l'unité dans $\mathbf{G}_{\Omega}\left(\mathbb{Z}_{p}\right)$.

\section{Opérateurs en $p$}

Dans la suite, nous notons souvent $\mathbf{M}_{\Omega}\left(\mathbb{Z}_{p}\right)^{*}$ l'un des groupes $\mathbf{M}_{\Omega}\left(\mathbb{Z}_{p}\right)$ ou son groupe dérivé $\mathbf{M}_{\Omega}\left(\mathbb{Z}_{p}\right)^{\prime}$, et $\mathbf{P}_{\Omega}\left(\mathbb{Z}_{p}\right)^{*}$ désigne, selon le cas, le sous-groupe $\mathbf{P}_{\Omega}\left(\mathbb{Z}_{p}\right)$ ou

$$
\mathbf{P}_{\Omega}\left(\mathbb{Z}_{p}\right)^{\prime}:=\mathbf{M}_{\Omega}\left(\mathbb{Z}_{p}\right)^{\prime} \ltimes \mathbf{U}_{\Omega}\left(\mathbb{Z}_{p}\right) .
$$

\subsection{Niveau infini}

Nous fixons une section $\sigma$ du morphisme

$$
\operatorname{RM}\left(\mathbb{Q}_{p}\right) \stackrel{\nu_{\mid \mathbf{R M}\left(\mathbb{Q}_{p}\right)}}{\longrightarrow} \Lambda_{\mathbf{M}} .
$$

Par compacité, $\mathbf{M}_{\Omega}\left(\mathbb{Z}_{p}\right) \cap \sigma\left(\Lambda_{\mathbf{M}}\right)=\{1\}$.

On pose $C:=\mathbf{M}_{\Omega}\left(\mathbb{Z}_{p}\right) / \mathbf{M}_{\Omega}\left(\mathbb{Z}_{p}\right)^{\prime}$.

Proposition 3.1.1. - Dans cette situation,

$$
D_{1, p}:=\sigma\left(\Lambda_{\mathbf{M}}^{+}\right) \mathbf{P}_{\Omega}\left(\mathbb{Z}_{p}\right)^{\prime} \subset D_{0, p}:=\sigma\left(\Lambda_{\mathbf{M}}^{+}\right) \mathbf{P}_{\Omega}\left(\mathbb{Z}_{p}\right)
$$


sont des sous-monoïdes de $\mathbf{P}\left(\mathbb{Q}_{p}\right)$.

De plus nous avons les isomorphismes d'anneaux suivants :

$$
\begin{aligned}
\mathcal{H}\left(\mathbf{P}_{\Omega}\left(\mathbb{Z}_{p}\right)^{\prime}, D_{1, p}\right) & \leftarrow \mathbb{Z}\left[\Lambda_{\mathbf{M}}^{+}\right] \\
\mathcal{H}\left(\mathbf{P}_{\Omega}\left(\mathbb{Z}_{p}\right)^{\prime}, D_{0, p}\right) & \leftarrow \mathbb{Z}\left[\Lambda_{\mathbf{M}}^{+}\right][C] \\
\mathcal{H}\left(\mathbf{P}_{\Omega}\left(\mathbb{Z}_{p}\right), D_{0, p}\right) & \simeq \mathbb{Z}\left[\Lambda_{\mathbf{M}}^{+}\right] .
\end{aligned}
$$

Enfin, $\mathbf{P}_{\Omega}\left(\mathbb{Z}_{p}\right)^{\prime}$ est un sous-groupe distingué, au sens du paragraphe 1.2 , de la paire $\mathbf{P}_{\Omega}\left(\mathbb{Z}_{p}\right) \subset D_{0, p}$.

Démonstration. - Les relations (12) montrent, d'une part, que

$$
D_{1, p} \subset D_{0, p} \subset \mathbf{P}\left(\mathbb{Q}_{p}\right)
$$

sont des sous-monoïdes, et de l'autre, que pour tous $\xi, \eta \in D_{0, p}$ :

$$
\begin{aligned}
\# & \left(\mathbf{P}_{\Omega}\left(\mathbb{Z}_{p}\right)^{*} \backslash \mathbf{P}_{\Omega}\left(\mathbb{Z}_{p}\right)^{*} \xi \eta \mathbf{P}_{\Omega}\left(\mathbb{Z}_{p}\right)^{*}\right) \\
& =\left(\mathbf{P}_{\Omega}\left(\mathbb{Z}_{p}\right)^{*}: \mathbf{P}_{\Omega}\left(\mathbb{Z}_{p}\right)^{*} \cap\left(\mathbf{P}_{\Omega}\left(\mathbb{Z}_{p}\right)^{*}\right)^{\xi \eta}\right) \\
& =\left(\mathbf{P}_{\Omega}\left(\mathbb{Z}_{p}\right)^{*}:\left(\mathbf{P}_{\Omega}\left(\mathbb{Z}_{p}\right)^{*}\right)^{\xi \eta}\right) \\
& =\left(\mathbf{P}_{\Omega}\left(\mathbb{Z}_{p}\right)^{*}:\left(\mathbf{P}_{\Omega}\left(\mathbb{Z}_{p}\right)^{*}\right)^{\eta}\right)\left(\left(\mathbf{P}_{\Omega}\left(\mathbb{Z}_{p}\right)^{*}\right)^{\eta}:\left(\mathbf{P}_{\Omega}\left(\mathbb{Z}_{p}\right)^{*}\right)^{\xi \eta}\right) \\
& =\left(\mathbf{P}_{\Omega}\left(\mathbb{Z}_{p}\right)^{*}:\left(\mathbf{P}_{\Omega}\left(\mathbb{Z}_{p}\right)^{*}\right)^{\eta}\right)\left(\mathbf{P}_{\Omega}\left(\mathbb{Z}_{p}\right)^{*}:\left(\mathbf{P}_{\Omega}\left(\mathbb{Z}_{p}\right)^{*}\right)^{\xi}\right) .
\end{aligned}
$$

D'où la multiplicativité nécessaire pour appliquer le corollaire 1.4.1. De plus, $\mathbf{P}_{\Omega}\left(\mathbb{Z}_{p}\right)$ étant un sous-groupe ouvert de $\mathbf{P}\left(\mathbb{Q}_{p}\right)$, tous ces indices sont finis. Ainsi l'anneau de Hecke $\mathcal{H}\left(\mathbf{P}_{\Omega}\left(\mathbb{Z}_{p}\right)^{*}, D_{0, p}\right)$ est isomorphe à l'algèbre du monoïde $\mathbf{P}_{\Omega}\left(\mathbb{Z}_{p}\right)^{*} \backslash D_{0, p} / \mathbf{P}_{\Omega}\left(\mathbb{Z}_{p}\right)^{*}$.

En utilisant (12) puis $\mathbf{M}_{\Omega}\left(\mathbb{Z}_{p}\right) \cap \sigma\left(\Lambda_{\mathbf{M}}\right)=\{1\}$, ce dernier est isomorphe à

$$
\begin{aligned}
D_{0, p} / \mathbf{P}_{\Omega}\left(\mathbb{Z}_{p}\right)^{*} & =\sigma\left(\Lambda_{\mathbf{M}}^{+}\right) \mathbf{M}_{\Omega}\left(\mathbb{Z}_{p}\right) / \mathbf{M}_{\Omega}\left(\mathbb{Z}_{p}\right)^{*} \\
& = \begin{cases}\Lambda_{\mathbf{M}}^{+} & \text {si } \mathbf{P}_{\Omega}\left(\mathbb{Z}_{p}\right)^{*}=\mathbf{P}_{\Omega}\left(\mathbb{Z}_{p}\right), \\
\Lambda_{\mathbf{M}}^{+} \times C & \text { si } \mathbf{P}_{\Omega}\left(\mathbb{Z}_{p}\right)^{*}=\mathbf{P}_{\Omega}\left(\mathbb{Z}_{p}\right)^{\prime} .\end{cases}
\end{aligned}
$$

De plus, le sous-anneau $\mathcal{H}\left(\mathbf{P}_{\Omega}\left(\mathbb{Z}_{p}\right)^{\prime}, D_{1, p}\right) \subset \mathcal{H}\left(\mathbf{P}_{\Omega}\left(\mathbb{Z}_{p}\right)^{\prime}, D_{0, p}\right)$ s'identifie immédiatement à l'algèbre du monoïde commutatif libre $\Lambda_{\mathbf{M}}^{+}$.

La dernière assertion signifie que les applications

$$
\begin{gathered}
\mathbf{P}_{\Omega}\left(\mathbb{Z}_{p}\right)^{\prime} \backslash \mathbf{P}_{\Omega}\left(\mathbb{Z}_{p}\right)^{\prime} \xi \mathbf{P}_{\Omega}\left(\mathbb{Z}_{p}\right)^{\prime} \\
{ }^{\downarrow} \\
\mathbf{P}_{\Omega}\left(\mathbb{Z}_{p}\right) \backslash \mathbf{P}_{\Omega}\left(\mathbb{Z}_{p}\right) \xi \mathbf{P}_{\Omega}\left(\mathbb{Z}_{p}\right)
\end{gathered}
$$

sont injectives $\left(\xi \in D_{0, p}\right)$, i.e.

$$
\forall \xi \in D_{0, p}, \quad \mathbf{P}_{\Omega}\left(\mathbb{Z}_{p}\right)^{\prime} \xi \mathbf{P}_{\Omega}\left(\mathbb{Z}_{p}\right)^{\prime} \cap \mathbf{P}_{\Omega}\left(\mathbb{Z}_{p}\right) \xi=\mathbf{P}_{\Omega}\left(\mathbb{Z}_{p}\right)^{\prime} \xi
$$

Ce qui est le cas puisque cette égalité est équivalente à celle projetée dans $\mathbf{M}$ et cette dernière vient du fait que $\sigma\left(\Lambda_{\mathbf{M}}^{+}\right) \mathbf{M}_{\Omega}\left(\mathbb{Z}_{p}\right)$ normalise $\mathbf{M}_{\Omega}\left(\mathbb{Z}_{p}\right)^{\prime}$. 


\subsection{Niveau fini}

Nous nous intéressons maintenant au niveau fini, i.e. aux sous-groupes de niveau de la forme $U \mathbf{P}_{\Omega}\left(\mathbb{Z}_{p}\right)$ et $U \mathbf{P}_{\Omega}\left(\mathbb{Z}_{p}\right)^{\prime}$, où $U \in \mathcal{T}_{\mathrm{bp}}$. Les premiers sont de type $\Gamma_{0}$ tandis que les seconds sont de type $\Gamma_{1}$.

Le groupe abélien profini $C$ s'identifie à la limite projective, indexée par $\mathcal{T}_{\mathrm{bp}}$, des groupes finis

$$
C_{U}:=\mathbf{M}_{\Omega}\left(\mathbb{Z}_{p}\right) /\left(\left(U \cap \mathbf{M}_{\Omega}\left(\mathbb{Z}_{p}\right)\right) \mathbf{M}_{\Omega}\left(\mathbb{Z}_{p}\right)^{\prime}\right) \quad\left(U \in \mathcal{T}_{\mathrm{bp}}\right) .
$$

Proposition 3.2.1. - Soit $U \in \mathcal{T}_{\mathrm{bp}}$. Alors $U D_{1, p} \subset U D_{0, p}$ sont des sous-monoüdes de $\mathbf{G}\left(\mathbb{Q}_{p}\right)$.

De plus, on a les isomorphismes d'anneaux suivants :

$$
\begin{aligned}
\mathcal{H}\left(U \mathbf{P}_{\Omega}\left(\mathbb{Z}_{p}\right)^{\prime}, U D_{1, p}\right) & \simeq \mathbb{Z}\left[\Lambda_{\mathbf{M}}^{+}\right] \\
\mathcal{H}\left(U \mathbf{P}_{\Omega}\left(\mathbb{Z}_{p}\right)^{\prime}, U D_{0, p}\right) & \simeq \mathbb{Z}\left[\Lambda_{\mathbf{M}}^{+}\right]\left[C_{U}\right] \\
\mathcal{H}\left(U \mathbf{P}_{\Omega}\left(\mathbb{Z}_{p}\right), U D_{0, p}\right) & \simeq \mathbb{Z}\left[\Lambda_{\mathbf{M}}^{+}\right] .
\end{aligned}
$$

De plus, $U \mathbf{P}_{\Omega}\left(\mathbb{Z}_{p}\right)^{\prime}$ est un sous-groupe distingué de la paire

$$
U \mathbf{P}_{\Omega}\left(\mathbb{Z}_{p}\right) \subset U D_{0, p}
$$

Démonstration. - Remarquons d'abord que $U \mathbf{P}_{\Omega}\left(\mathbb{Z}_{p}\right)^{*} \subset \mathbf{G}_{\Omega}\left(\mathbb{Z}_{p}\right)$ est un sous-groupe car $U \triangleleft \mathbf{G}_{\Omega}\left(\mathbb{Z}_{p}\right)$ est distingué.

Établissons ensuite, pour $\xi \in \sigma\left(\Lambda_{\mathbf{M}}^{+}\right)$et $p \in \mathbf{P}_{\Omega}\left(\mathbb{Z}_{p}\right)$, les égalités :

$$
\begin{aligned}
U \mathbf{P}_{\Omega}\left(\mathbb{Z}_{p}\right)^{*} \xi p U \mathbf{P}_{\Omega}\left(\mathbb{Z}_{p}\right)^{*} & =U \mathbf{P}_{\Omega}\left(\mathbb{Z}_{p}\right)^{*} \xi p \mathbf{P}_{\Omega}\left(\mathbb{Z}_{p}\right)^{*} \\
& =\left(U \cap \mathbf{P}_{\Omega}^{-}\left(\mathbb{Z}_{p}\right)\right) \xi p \mathbf{P}_{\Omega}\left(\mathbb{Z}_{p}\right)^{*} \\
& =\left(U \cap \mathbf{U}_{\Omega}^{-}\left(\mathbb{Z}_{p}\right)\right) \xi p\left(U \cap \mathbf{M}_{\Omega}\left(\mathbb{Z}_{p}\right)\right) \mathbf{P}_{\Omega}\left(\mathbb{Z}_{p}\right)^{*} .
\end{aligned}
$$

La première égalité vient de $p U=U p$, de la décomposition (13) et de (14). La seconde de la décomposition (13) et de (12). Et la troisième, toujours de la décomposition (13).

L'égalité (16) montre que $U D_{1, p} \subset U D_{0, p}$ sont des sous-monoïdes de $\mathbf{G}\left(\mathbb{Q}_{p}\right)$ et que le morphisme

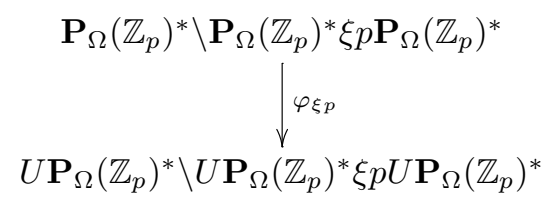

est surjectif. Il est aussi injectif car

$$
\mathbf{P}_{\Omega}\left(\mathbb{Z}_{p}\right)^{*} \xi p \mathbf{P}_{\Omega}\left(\mathbb{Z}_{p}\right)^{*} \cap U \mathbf{P}_{\Omega}\left(\mathbb{Z}_{p}\right)^{*} \xi p=\mathbf{P}_{\Omega}\left(\mathbb{Z}_{p}\right)^{*} \xi p
$$

En effet, cette égalité est à nouveau équivalente à celle projetée dans $\mathbf{M}$, laquelle résulte du fait que $\sigma\left(\Lambda_{\mathbf{M}}^{+}\right) \mathbf{M}_{\Omega}\left(\mathbb{Z}_{p}\right)$ normalise $\mathbf{M}_{\Omega}\left(\mathbb{Z}_{p}\right)^{*}$.

Comme dans la preuve de la proposition 3.1.1, nous disposons donc de l'hypothèse de multiplicativité nécessaire à l'application du corollaire 1.4.1: l'anneau de Hecke $\mathcal{H}\left(U \mathbf{P}_{\Omega}\left(\mathbb{Z}_{p}\right)^{*}, U D_{0, p}\right)$ est isomorphe à l'algèbre du monoïde $U \mathbf{P}_{\Omega}\left(\mathbb{Z}_{p}\right)^{*} \backslash U D_{0, p} / U \mathbf{P}_{\Omega}\left(\mathbb{Z}_{p}\right)^{*}$.

En utilisant (17) puis $\mathbf{M}_{\Omega}\left(\mathbb{Z}_{p}\right) \cap \sigma\left(\Lambda_{\mathbf{M}}\right)=\{1\}$, ce dernier est isomorphe à 


$$
\begin{aligned}
& D_{0, p} /\left(\left(U \cap \mathbf{M}_{\Omega}\left(\mathbb{Z}_{p}\right)\right) \mathbf{P}_{\Omega}\left(\mathbb{Z}_{p}\right)^{*}\right) \\
& =\sigma\left(\Lambda_{\mathbf{M}}^{+}\right) \mathbf{M}_{\Omega}\left(\mathbb{Z}_{p}\right) /\left(\left(U \cap \mathbf{M}_{\Omega}\left(\mathbb{Z}_{p}\right)\right) \mathbf{M}_{\Omega}\left(\mathbb{Z}_{p}\right)^{*}\right) \\
& \quad= \begin{cases}\Lambda_{\mathbf{M}}^{+} & \text {si } \mathbf{P}_{\Omega}\left(\mathbb{Z}_{p}\right)^{*}=\mathbf{P}_{\Omega}\left(\mathbb{Z}_{p}\right), \\
\Lambda_{\mathbf{M}}^{+} \times C_{U} & \text { si } \mathbf{P}_{\Omega}\left(\mathbb{Z}_{p}\right)^{*}=\mathbf{P}_{\Omega}\left(\mathbb{Z}_{p}\right)^{\prime} .\end{cases}
\end{aligned}
$$

De plus, le sous-anneau

$$
\mathcal{H}\left(U \mathbf{P}_{\Omega}\left(\mathbb{Z}_{p}\right)^{\prime}, U D_{1, p}\right) \subset \mathcal{H}\left(U \mathbf{P}_{\Omega}\left(\mathbb{Z}_{p}\right)^{\prime}, U D_{0, p}\right)
$$

s'identifie immédiatement à l'algèbre du monoïde commutatif libre $\Lambda_{\mathbf{M}}^{+}$.

La dernière assertion résulte des isomorphismes $\varphi_{\xi p}$ et des injections (15).

\subsection{Conséquence de la propriété de contraction}

La propriété de contraction des éléments $t \in \mathbf{R M}\left(\mathbb{Q}_{p}\right)^{+}$, décrite au lemme 2.4.1 se traduit, sur les opérateurs de Hecke, par les relations suivantes :

LEMME 3.3.1. - Soient $U, U^{\prime} \in \mathcal{T}_{\mathrm{bp}}$ tels que $U^{\prime} \subset U$.

Il existe $t \in \mathbf{R M}\left(\mathbb{Q}_{p}\right)^{+}$tel que $\left(U^{\prime} \cap \mathbf{U}_{\Omega}^{-}\left(\mathbb{Z}_{p}\right)\right)^{t} \supset U \cap \mathbf{U}_{\Omega}^{-}\left(\mathbb{Z}_{p}\right)$. Pour un tel $t$, on a les relations :

$$
\begin{aligned}
& U^{\prime} \mathbf{P}_{\Omega}\left(\mathbb{Z}_{p}\right) t U \mathbf{P}_{\Omega}\left(\mathbb{Z}_{p}\right) \mid U \mathbf{P}_{\Omega}\left(\mathbb{Z}_{p}\right) 1 U^{\prime} \mathbf{P}_{\Omega}\left(\mathbb{Z}_{p}\right)=U^{\prime} \mathbf{P}_{\Omega}\left(\mathbb{Z}_{p}\right) t U^{\prime} \mathbf{P}_{\Omega}\left(\mathbb{Z}_{p}\right), \\
& U \mathbf{P}_{\Omega}\left(\mathbb{Z}_{p}\right) 1 U^{\prime} \mathbf{P}_{\Omega}\left(\mathbb{Z}_{p}\right) \mid U^{\prime} \mathbf{P}_{\Omega}\left(\mathbb{Z}_{p}\right) t U \mathbf{P}_{\Omega}\left(\mathbb{Z}_{p}\right)=U \mathbf{P}_{\Omega}\left(\mathbb{Z}_{p}\right) t U \mathbf{P}_{\Omega}\left(\mathbb{Z}_{p}\right) .
\end{aligned}
$$

Démonstration. - D'après le lemme 2.4.1, les sous-groupes ${ }^{t}\left(U \cap \mathbf{U}_{\Omega}^{-}\left(\mathbb{Z}_{p}\right)\right)$, lorsque $t$ varie dans $\mathbf{R M}\left(\mathbb{Q}_{p}\right)^{+}$, forment un système fondamental de voisinage de l'unité dans $\mathbf{U}^{-}\left(\mathbb{Q}_{p}\right)$. D'où la première assertion.

Puisque $U^{\prime} \subset U$, on a

$$
\#\left(\left(U \mathbf{P}_{\Omega}\left(\mathbb{Z}_{p}\right)\right) \backslash U \mathbf{P}_{\Omega}\left(\mathbb{Z}_{p}\right) 1 U^{\prime} \mathbf{P}_{\Omega}\left(\mathbb{Z}_{p}\right)\right)=1
$$

Ainsi, selon la proposition 1.4.1, il suffit, pour établir les deux égalités d'avoir l'égalité et l'isomorphisme suivants :

$$
\begin{gathered}
U^{\prime} \mathbf{P}_{\Omega}\left(\mathbb{Z}_{p}\right) t U \mathbf{P}_{\Omega}\left(\mathbb{Z}_{p}\right)=U^{\prime} \mathbf{P}_{\Omega}\left(\mathbb{Z}_{p}\right) t U^{\prime} \mathbf{P}_{\Omega}\left(\mathbb{Z}_{p}\right), \\
\left(U^{\prime} \mathbf{P}_{\Omega}\left(\mathbb{Z}_{p}\right)\right) \backslash U^{\prime} \mathbf{P}_{\Omega}\left(\mathbb{Z}_{p}\right) t U \mathbf{P}_{\Omega}\left(\mathbb{Z}_{p}\right) \stackrel{\sim}{\rightarrow}\left(U \mathbf{P}_{\Omega}\left(\mathbb{Z}_{p}\right)\right) \backslash U \mathbf{P}_{\Omega}\left(\mathbb{Z}_{p}\right) t U \mathbf{P}_{\Omega}\left(\mathbb{Z}_{p}\right) .
\end{gathered}
$$

En utilisant la décomposition (14) puis la condition sur $t$, on a

$$
\begin{aligned}
U^{\prime} \mathbf{P}_{\Omega}\left(\mathbb{Z}_{p}\right) t U \mathbf{P}_{\Omega}\left(\mathbb{Z}_{p}\right) & =U^{\prime} \mathbf{P}_{\Omega}\left(\mathbb{Z}_{p}\right) t\left(U \cap \mathbf{U}_{\Omega}^{-}\left(\mathbb{Z}_{p}\right)\right) \mathbf{P}_{\Omega}\left(\mathbb{Z}_{p}\right) \\
& =U^{\prime} \mathbf{P}_{\Omega}\left(\mathbb{Z}_{p}\right) t \mathbf{P}_{\Omega}\left(\mathbb{Z}_{p}\right),
\end{aligned}
$$

d'où l'égalité (19).

Les isomorphismes $\varphi_{\xi p}$ de (18) établissent l'injectivité du morphisme (20). Sa surjectivité est évidente. 


\section{Cohomologie des variétés modulaires}

Dans cette section, nous comparons la cohomologie de la variété modulaire $\mathcal{M}$ d'un groupe réductif connexe $\mathbf{G}$ sur $\mathbb{Q}$, en niveau fini $K$, à la cohomologie du groupe $K$. Nous aboutissons à un analogue adélique de la comparaison de la cohomologie d'un groupe $\Gamma$ avec celle d'un espace $\mathrm{K}(\Gamma, 1)$.

Nous parlons de niveau fini pour signifier que $K$ est un sous-groupe ouvert compact du groupe des points adéliques finis $\mathbf{G}\left(\mathbb{A}_{\mathrm{f}}\right)$. Le résultat de comparaison que nous démontrons (cf. prop. 4.3.2) met en jeu la cohomologie du groupe $K$ muni de la topologie discrète.

Dans la suite, l'application principale de ce résultat sera la suite spectrale de changement de niveau du théorème 5.2.1.

\subsection{Variétés modulaires}

Nous envisageons ici les variétés modulaires uniquement d'un point de vue topologique, en tant qu'adélisation des espaces symétriques associés aux groupes réductifs.

\subsubsection{Domaine symétrique}

Soit $\mathbf{R G}$ le radical de $\mathbf{G}$, c'est-à-dire le plus grand sous-tore central. Lorsque $k=\mathbb{Q}$ ou $\mathbb{R}$, on note $\mathbf{R}_{k} \mathbf{G}$ le plus grand sous-tore $k$-déployé de $\mathbf{R G}$ et $\mathbf{R}_{k} \mathbf{G}(\mathbb{R})^{+}$la composante neutre du groupe de ses $\mathbb{R}$-points.

Nous notons $X$ le domaine symétrique associé au groupe $\mathbf{G}$. C'est un espace homogène sous $\mathbf{G}(\mathbb{R})$ dont les fixateurs sont de la forme $K_{\mathbb{R}} \cdot \mathbf{R}_{\mathbb{Q}} \mathbf{G}(\mathbb{R})$ où $K_{\mathbb{R}}$ est sous-groupe compact maximal de $\mathbf{G}(\mathbb{R})$. Cette description détermine $X$ à isomorphisme près. On le munit de la topologie quotient de $\mathbf{G}(\mathbb{R})$. L'action de $\mathbf{G}(\mathbb{R})$ sur $X$ se factorise en une action propre du quotient $\mathbf{G}(\mathbb{R}) / \mathbf{R}_{\mathbb{Q}} \mathbf{G}(\mathbb{R})^{+}$.

L'espace symétrique $X^{\text {ad }}$ du groupe adjoint $\mathbf{G}^{\text {ad }}$ de $\mathbf{G}$ s'identifie à l'ensemble des sousgroupes compacts maximaux $K_{\mathbb{R}} \subset \mathbf{G}(\mathbb{R})$ car leur normalisateur est $K_{\mathbb{R}} \cdot \mathbf{R}_{\mathbb{R}} \mathbf{G}(\mathbb{R})$.

La décomposition $\mathbf{G}(\mathbb{R})=\mathbf{R}_{\mathbb{R}} \mathbf{G}(\mathbb{R})^{+} \times\left(\mathbf{G}(\mathbb{R}) / \mathbf{R}_{\mathbb{R}} \mathbf{G}(\mathbb{R})^{+}\right)$permet d'identifier $X$ avec le produit $\left(\mathbf{R}_{\mathbb{R}} \mathbf{G}(\mathbb{R})^{+} / \mathbf{R}_{\mathbb{Q}} \mathbf{G}(\mathbb{R})^{+}\right) \times X^{\text {ad }}$.

\subsubsection{Variété modulaire de niveau fini}

Si $K \subset \mathbf{G}\left(\mathbb{A}_{\mathrm{f}}\right)$ est un sous-groupe ouvert compact, la variété modulaire de $\mathbf{G}$ de niveau $K$ est

$$
\mathcal{M}_{K}:=\mathbf{G}(\mathbb{Q}) \backslash X \times \mathbf{G}\left(\mathbb{A}_{\mathrm{f}}\right) / K
$$

où les actions sont définies par $\gamma(x, g) k=(\gamma x, \gamma g k)$, quels que soient $\gamma \in \mathbf{G}(\mathbb{Q}), x \in X$, $g \in \mathbf{G}\left(\mathbb{A}_{\mathrm{f}}\right), k \in K$.

Puisque $X$ est connexe, l'ensemble des composantes connexes de $\mathcal{M}_{K}$ est $\mathbf{G}(\mathbb{Q}) \backslash \mathbf{G}\left(\mathbb{A}_{\mathrm{f}}\right) / K$. Plus précisément, si pour tout sous-groupe $H \subset \mathbf{G}\left(\mathbb{A}_{\mathrm{f}}\right)$, on note $\Gamma_{H}:=\mathbf{G}(\mathbb{Q}) \cap H$, alors la composante connexe correspondant à la double classe $\mathbf{G}(\mathbb{Q}) g K,\left(g \in \mathbf{G}\left(\mathbb{A}_{\mathrm{f}}\right)\right)$, s'identifie au quotient $\Gamma_{g_{K}} \backslash X$. L'identification et la compatibilité entre deux éléments de la même double 
classe sont données par le diagramme commutatif

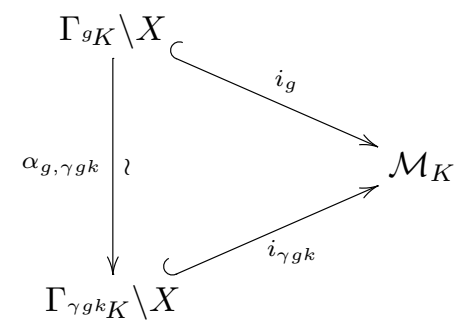

où pour $g \in \mathbf{G}\left(\mathbb{A}_{\mathrm{f}}\right), \gamma \in \mathbf{G}(\mathbb{Q}), k \in K$ et $x \in X$, on a

$$
i_{g}\left(\Gamma_{g_{K}} x\right)=\mathbf{G}(\mathbb{Q})(x, g) K \quad \text { et } \quad \alpha_{g, \gamma g k}\left(\Gamma_{g_{K}} x\right)=\Gamma_{\gamma g^{k} K} \gamma x .
$$

\subsection{Compactification de Borel-Serre}

Nous décrivons ici l'élargissement $\bar{X}$, du domaine symétrique $X$, défini par Borel et Serre [5].

\subsubsection{Sous-groupes paraboliques}

Soient $\mathfrak{P}$ l'ensemble des $\mathbb{Q}$-sous-groupes paraboliques de $\mathbf{G}$ et $\mathfrak{P}_{1} \subset \mathfrak{P}$ le sous-ensemble des $\mathbb{Q}$-sous-groupes paraboliques propres maximaux.

Pour $\mathbf{P} \in \mathfrak{P}$, on définit le sous-ensemble (fini) $s(\mathbf{P}) \subset \mathfrak{P}_{1}$ des $\mathbb{Q}$-sous-groupes paraboliques propres maximaux contenant $\mathbf{P}$. C'est aussi l'unique sous-ensemble $S \subset \mathfrak{P}_{1}$ tel que

$$
\mathbf{P}=\bigcap_{\mathbf{P}_{1} \in S} \mathbf{P}_{1}
$$

On note $\mathfrak{P}_{i} \subset \mathfrak{P}$ le sous-ensemble des $\mathbb{Q}$-sous-groupes paraboliques $\mathbf{P}$ de $\mathbb{Q}$-rang parabolique $i$, c'est-à-dire tels que $s(\mathbf{P})$ est de cardinal $i$.

L'application $s$ passe au quotient par l'action par conjugaison (à gauche pour fixer les idées) du groupe $\mathbf{G}(\mathbb{Q})$. Pour une classe de $\mathbf{G}(\mathbb{Q})$-conjugaison $c$ de $\mathbb{Q}$-sous-groupes paraboliques, on note aussi $s(c) \subset \mathfrak{P}_{1}$ l'ensemble des $\mathbb{Q}$-sous-groupes paraboliques propres maximaux contenant un élément de la classe $c$.

\subsection{2. Élargissement du domaine symétrique}

Pour chaque $\mathbf{P} \in \mathfrak{P}_{i}$ de radical unipotent $\mathbf{U}$, soient $\mathbf{S}_{\mathbf{P}}$ le radical $\mathbb{Q}$-déployé de $\mathbf{P} / \mathbf{U}$ et $A_{\mathbf{P}} \simeq\left(\mathbb{R}_{+}^{*}\right)^{i}$ la composante neutre du groupe $\left(\mathbf{S}_{\mathbf{P}} / \mathbf{S}_{\mathbf{G}}\right)(\mathbb{R})$. Si $\mathbf{P}^{\prime} \in \mathfrak{P}$ contient $\mathbf{P}$, alors l'injection naturelle $\mathbf{S}_{\mathbf{P}^{\prime}} \subset \mathbf{S}_{\mathbf{P}}$ induit une injection $a_{\mathbf{P}^{\prime}, \mathbf{P}}: A_{\mathbf{P}^{\prime}} \hookrightarrow A_{\mathbf{P}}$. Ce qui permet l'identification $A_{\mathbf{P}}=\prod_{\mathbf{P}_{1} \in s(\mathbf{P})} A_{\mathbf{P}_{1}}$.

Si $\mathbf{P}_{1} \in \mathfrak{P}_{1}$, on forme l'union disjointe $\overline{A_{\mathbf{P}_{1}}}:=A_{\mathbf{P}_{1}} \amalg\{0\}$, munie de l'action de $A_{\mathbf{P}_{1}}$ qui laisse fixe 0 et prolonge celle sur lui-même par translation. Plus généralement, pour $\mathbf{P} \in \mathfrak{P}_{i}$, on pose $\overline{A_{\mathbf{P}}}:=\prod_{\mathbf{P}_{1} \in s(\mathbf{P})} \overline{A_{\mathbf{P}_{1}}} \simeq\left(\mathbb{R}_{+}\right)^{i}$, muni de l'action diagonale. Alors on a la décomposition

$$
\overline{A_{\mathbf{P}}}=\coprod_{\mathbf{P}^{\prime} \supset \mathbf{P}}\left(\prod_{\mathbf{P}_{1} \in s(\mathbf{P}) \backslash s\left(\mathbf{P}^{\prime}\right)} A_{\mathbf{P}_{1}} \times \prod_{\mathbf{P}_{1} \in s\left(\mathbf{P}^{\prime}\right)}\{0\}\right) .
$$

Borel et Serre définissent une action géodésique [5, §3.2], notée ici à droite, de $A_{\mathbf{P}}$ sur $X$. Le coin associé à $\mathbf{P}$ est le produit contracté $X(\mathbf{P}):=X \times{ }^{A_{\mathbf{P}}} \overline{A_{\mathbf{P}}}$. Si on note $e(\mathbf{P}):=X / A_{\mathbf{P}}$, la décomposition précédente de $\overline{A_{\mathbf{P}}}$ donne $X(\mathbf{P})=\coprod_{\mathbf{P}^{\prime} \supset \mathbf{P}} e\left(\mathbf{P}^{\prime}\right)$. 
La variété $\bar{X}$ est définie topologiquement comme la limite du système inductif $(X(\mathbf{P}))_{\mathbf{P} \in \mathfrak{P}}$ dont les applications de transition sont les immersions ouvertes $i d_{X} * a_{\mathbf{P}^{\prime}, \mathbf{P}}: X\left(\mathbf{P}^{\prime}\right) \hookrightarrow X(\mathbf{P})$, où $\mathbf{P} \subset \mathbf{P}^{\prime}$. Du point de vue ensembliste, on a $\bar{X}=\coprod_{\mathbf{P} \in \mathfrak{P}} e(\mathbf{P})$ et l'adhérence $\overline{e(\mathbf{P})}$ de $e(\mathbf{P})$ dans $\bar{X}$ est $\overline{e(\mathbf{P})}=\coprod_{\mathbf{P}^{\prime} \subset \mathbf{P}} e\left(\mathbf{P}^{\prime}\right)$.

Ainsi $X=X(\mathbf{G})=e(\mathbf{G})$ s'identifie à un ouvert dense de $\bar{X}$. L'inclusion $X \subset \bar{X}$ est en fait une équivalence d'homotopie. D' après [5, 8.3], les fermés $\overline{e(\mathbf{P})}(\mathbf{P} \in \mathfrak{P})$ sont des variétés à bords contractiles. Le sous-espace $e(\mathbf{P}) \subset \bar{X}$ est de codimension le rang parabolique de $\mathbf{P}$. De plus le recouvrement du bord $\partial \bar{X}:=\bar{X} \backslash X$ par les fermés $\overline{e\left(\mathbf{P}_{1}\right)}\left(\mathbf{P}_{1} \in \mathfrak{P}_{1}\right)$ a pour nerf l'immeuble de Tits $[35,5.3]$ de $\mathbf{G}$.

\subsubsection{Prolongement de l'action}

Soient $\mathbf{P} \in \mathfrak{P}$ et $\gamma \in \mathbf{G}(\mathbb{Q})$. Puisque $A_{\mathbf{P}}$ est central dans $\mathbf{P} / \mathbf{U}$, l'isomorphisme de conjugaison $g \mapsto{ }^{\gamma} g$ induit un isomorphisme $A_{\mathbf{P}} \simeq A_{\gamma_{\mathbf{P}}}$ (encore noté $\gamma_{\text {. }}$ ) qui ne dépend que de $\mathbf{P}$ et ${ }^{\gamma} \mathbf{P}$ (pas de $\gamma$ ). De plus, l'action géodésique est telle que $\gamma(x a)=(\gamma x)\left({ }^{\gamma} a\right)$, pour tous $x \in X$ et $a \in A_{\mathbf{P}}$.

Par conséquent, l'action de $\mathbf{G}(\mathbb{Q})$ sur $X$ se prolonge à $\bar{X}$ en permutant les coins, ainsi que les bords :

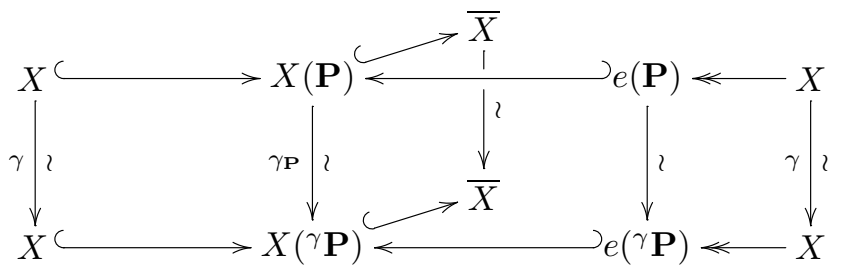

avec $\gamma_{\mathbf{P}}(x * t)=(\gamma x) *\left({ }^{\gamma} t\right)$ pour $x \in X, t \in \overline{A_{\mathbf{P}}}$.

\subsubsection{Compactification}

Soit $K \subset \mathbf{G}\left(\mathbb{A}_{\mathrm{f}}\right)$ un sous-groupe ouvert compact. La variété

$$
\overline{\mathcal{M}}_{K}:=\mathbf{G}(\mathbb{Q}) \backslash \bar{X} \times \mathbf{G}\left(\mathbb{A}_{\mathrm{f}}\right) / K
$$

a pour composantes connexes les quotients $\Gamma_{g_{K}} \backslash \bar{X}\left(g \in \mathbf{G}\left(\mathbb{A}_{\mathrm{f}}\right)\right)$, identifiés de la même façon qu'en 4.1.2, en remplaçant $X$ par $\bar{X}$.

Borel et Serre ont montré [5, th. 9.3] que l'action de $\Gamma_{g_{K}}$ sur $\bar{X}$ est propre et que le quotient $\Gamma_{g_{K}} \backslash \bar{X}$ est compact. Ainsi, la variété à bord $\overline{\mathcal{M}}_{K}$ est une compactification de $\mathcal{M}_{K}$, dont le bord est

$$
\partial \overline{\mathcal{M}}_{K}:=\overline{\mathcal{M}}_{K} \backslash \mathcal{M}_{K}=\mathbf{G}(\mathbb{Q}) \backslash \partial \bar{X} \times \mathbf{G}\left(\mathbb{A}_{\mathrm{f}}\right) / K
$$

\subsubsection{Décomposition du bord}

Soit $c$ la classe de $\mathbf{G}(\mathbb{Q})$-conjugaison de $\mathbf{P} \in \mathfrak{P}$. Puisque deux sous-groupes paraboliques conjugués distincts ne peuvent contenir un même sous-groupe parabolique et $\mathbf{P}$ est son propre normalisateur dans $\mathbf{G}$, on a

$$
\bigcup_{\mathbf{P}^{\prime} \in c} \overline{e\left(\mathbf{P}^{\prime}\right)}=\coprod_{\mathbf{P}^{\prime} \in c} \overline{e\left(\mathbf{P}^{\prime}\right)}=\mathbf{G}(\mathbb{Q}) \times{ }^{\mathbf{P}(\mathbb{Q})} \overline{e(\mathbf{P})} .
$$


Par conséquent, le fermé $\partial_{c} \overline{\mathcal{M}}_{K} \subset \partial \overline{\mathcal{M}}_{K}$ défini par

$$
\partial_{c} \overline{\mathcal{M}}_{K}:=\mathbf{G}(\mathbb{Q}) \backslash\left(\bigcup_{\mathbf{P}^{\prime} \in c} \overline{e\left(\mathbf{P}^{\prime}\right)}\right) \times \mathbf{G}\left(\mathbb{A}_{\mathrm{f}}\right) / K
$$

s'écrit aussi $\partial_{c} \overline{\mathcal{M}}_{K}=\mathbf{P}(\mathbb{Q}) \backslash \overline{e(\mathbf{P})} \times \mathbf{G}\left(\mathbb{A}_{\mathrm{f}}\right) / K$ et l'espace des composantes connexes de cette variété est

$$
\mathbf{P}(\mathbb{Q}) \backslash \mathbf{G}\left(\mathbb{A}_{\mathrm{f}}\right) / K
$$

Si, pour tout sous-groupe $H \subset \mathbf{G}\left(\mathbb{A}_{\mathrm{f}}\right)$, on note $\Gamma_{H}(\mathbf{P}):=\mathbf{P}(\mathbb{Q}) \cap H$, alors la composante connexe correspondant à la double classe $\mathbf{P}(\mathbb{Q}) g K\left(g \in \mathbf{G}\left(\mathbb{A}_{\mathrm{f}}\right)\right)$, s'identifie au quotient $\Gamma_{g_{K}}(\mathbf{P}) \backslash \overline{e(\mathbf{P})}$ avec les compatibilités données par le diagramme commutatif

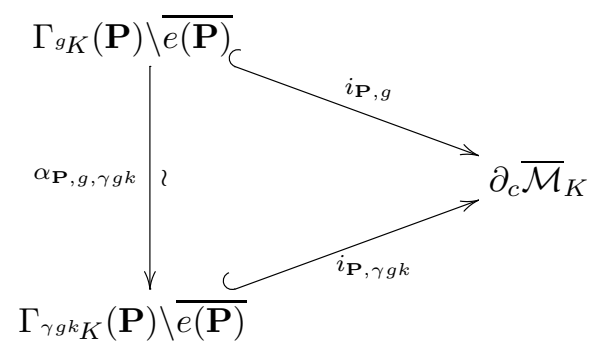

où pour $g \in \mathbf{G}\left(\mathbb{A}_{\mathrm{f}}\right), \gamma \in \mathbf{P}(\mathbb{Q}), k \in K$ et $x \in \overline{e(\mathbf{P})}$, on a

$$
\begin{aligned}
& i_{\mathbf{P}, g}\left(\Gamma_{g_{K}}(\mathbf{P}) x\right)=\mathbf{P}(\mathbb{Q})(x, g) K \quad \text { et } \\
& \alpha_{\mathbf{P}, g, \gamma g k}\left(\Gamma_{g_{K}}(\mathbf{P}) x\right)=\Gamma_{\gamma g k_{K}}(\mathbf{P}) \gamma x .
\end{aligned}
$$

Lorsque $c_{1}$ parcourt l'ensemble $\mathbf{G}(\mathbb{Q}) \backslash \mathfrak{P}_{1}$ des classes de conjugaison de $\mathbb{Q}$-sous-groupes paraboliques propres maximaux de $\mathbf{G}$, les fermés $\partial_{c_{1}} \overline{\mathcal{M}}$, forment un recouvrement localement fini du bord $\partial \overline{\mathcal{M}}_{K}$.

LEMME 4.2.1. - Pour tout sous-ensemble $S \subset \mathfrak{P}_{1}$ stable par conjugaison sous $\mathbf{G}(\mathbb{Q})$, on a

$$
\bigcap_{c_{1} \subset S} \partial_{c_{1}} \overline{\mathcal{M}}_{K}=\coprod_{s(c)=S} \partial_{c} \overline{\mathcal{M}}_{K}
$$

où $c_{1}$ et c désignent des classes de $\mathbf{G}(\mathbb{Q})$-conjugaisons de $\mathbb{Q}$-sous-groupes paraboliques de $\mathbf{G}$.

Rappelons que $s(c) \subset \mathfrak{P}_{1}$ est défini en 4.2.1 comme l'ensemble des $\mathbb{Q}$-sous-groupes paraboliques propres maximaux contenant un élément de la classe $c$.

Démonstration. - Par distributivité, on a

$$
\bigcap_{c_{1} \subset S} \bigcup_{\mathbf{P}_{1} \in c_{1}} \overline{e\left(\mathbf{P}_{1}\right)}=\bigcup_{\left(\mathbf{P}_{c_{1}}\right) \in \prod_{c_{1} \subset S} c_{1}} \bigcap_{c_{1} \subset S} \overline{e\left(\mathbf{P}_{c_{1}}\right)}
$$

où l'union à droite est indexée par les familles $\left(\mathbf{P}_{c_{1}}\right)_{c_{1} \subset S}$ constituées d'un représentant $\mathbf{P}_{c_{1}}$ de chaque classe $c_{1} \subset S$. 
Or, l'intersection de droite est non vide seulement lorsque l'intersection de la famille $\left(\mathbf{P}_{c_{1}}\right)_{c_{1} \subset S}$ correspondante est un $\mathbb{Q}$-sous-groupe parabolique $\mathbf{P}=\bigcap_{c_{1} \subset S} \mathbf{P}_{c_{1}}$, auquel cas elle vaut $\overline{e(\mathbf{P})}$. Mais un $\mathbb{Q}$-sous-groupe parabolique $\mathbf{P}$ est l'intersection d'une telle famille $\left(\mathbf{P}_{c_{1}}\right)_{c_{1} \subset S}$ si et seulement si $s(c(\mathbf{P}))=S$ car un sous-groupe parabolique ne peut être contenu dans deux sous-groupes paraboliques conjugués distincts. Pour cette dernière raison encore, on a finalement l'union disjointe dans la décomposition :

$$
\bigcap_{c_{1} \subset S} \bigcup_{\mathbf{P}_{1} \in c_{1}} \overline{e\left(\mathbf{P}_{1}\right)}=\coprod_{s(c)=S} \bigcup_{\mathbf{P} \in c} \overline{e(\mathbf{P})}
$$

Ce qui, après passage au quotient par $\mathbf{G}(\mathbb{Q})$ et $K$, donne la formule voulue.

\subsection{Cohomologie de niveau fini}

\subsubsection{Sous-groupes assez petits et espaces $K(\Gamma, 1)$}

Nous dirons qu'un sous-groupe $H$ de $\mathbf{G}\left(\mathbb{A}_{\mathrm{f}}\right)$ est assez petit s'il vérifie la condition (TF) les groupes $\Gamma_{g_{H}}=\mathbf{G}(\mathbb{Q}) \cap H$ (pour $g \in \mathbf{G}\left(\mathbb{A}_{\mathrm{f}}\right)$ ) n'ont pas de torsion.

LEMME 4.3.1. - L'ensemble $\mathcal{T}$ des sous-groupes ouverts $K \subset \mathbf{G}\left(\mathbb{A}_{\mathrm{f}}\right)$ compacts assez petits au sens de $(T F)$ est un système fondamental de voisinages de l'unité dans $\mathbf{G}\left(\mathbb{A}_{\mathrm{f}}\right)$.

Pour un tel sous-groupe $K$, chaque composante connexe $\Gamma_{g_{K}} \backslash X$ de la variété modulaire $\mathcal{M}_{K}$ de niveau $K$ est un espace $\mathrm{K}\left(\Gamma_{g_{K}}, 1\right)$.

Démonstration. - Puisque $\mathbf{G}\left(\mathbb{A}_{\mathrm{f}}\right)$ est un groupe localement profini, ses sous-groupes ouverts compacts forment un système fondamental de voisinage de l'unité ( $c f$. [8, III, p. 36, cor. 1]). L'existence de sous-groupes ouverts compacts $K$ tels que les $\Gamma_{g_{K}}$ n'ont pas de torsion est démontré dans [4]. L'ensemble $\mathcal{T}$ forme donc bien un système fondamental de voisinages de l'unité.

Soient $K \in \mathcal{T}$ et $g \in \mathbf{G}\left(\mathbb{A}_{\mathrm{f}}\right)$. Puisque $\mathbf{R}_{\mathbb{Q}} \mathbf{G}$ est un tore déployé sur $\mathbb{Q}$, ses sous-groupes arithmétiques sont finis. Le groupe $\mathbf{R}_{\mathbb{Q}} \mathbf{G}(\mathbb{R})^{+}$étant sans torsion, on a donc

$$
\Gamma_{g_{K}} \cap \mathbf{R}_{\mathbb{Q}} \mathbf{G}(\mathbb{R})^{+}=\{1\} .
$$

Ainsi, $\Gamma_{g_{K}}$ s'identifie à un sous-groupe discret et sans torsion (par définition de $\mathcal{T}$ ) de $\mathbf{G}(\mathbb{R}) / \mathbf{R}_{\mathbb{Q}} \mathbf{G}(\mathbb{R})^{+}$. Ce dernier agissant proprement sur $X$, selon [8, III, p. 32, prop. 8], $\Gamma_{g_{K}}$ agit proprement, mais aussi librement, sur $X$. Puisque $X$ est contractile (car homéomorphe à un espace euclidien), nous en concluons que $\Gamma_{g_{K}} \backslash X$ est un espace $\mathrm{K}\left(\Gamma_{g_{K}}, 1\right)$.

\subsubsection{Systèmes locaux}

Soit $K \in \mathcal{T}$. À tout $\mathbb{Z}[K]$-module $L$, nous associons le fibré

$$
\mathcal{M}_{K}(L):=\mathbf{G}(\mathbb{Q}) \backslash X \times \mathbf{G}\left(\mathbb{A}_{\mathrm{f}}\right) \times{ }^{K} L
$$

sur $\mathcal{M}_{K}$, où les actions sont définies par $\gamma(x, g * l)=(\gamma x,(\gamma g) * l)$, quels que soient $\gamma \in \mathbf{G}(\mathbb{Q})$, $x \in X, g \in \mathbf{G}\left(\mathbb{A}_{\mathrm{f}}\right)$ et $l \in L$.

Si nous faisons agir les groupes $\Gamma_{g_{K}}$ sur $L$ par $(\gamma, l) \mapsto \gamma^{g} . l$, quels que soient $\gamma \in \Gamma_{g_{K}}, l \in L$ et $g \in \mathbf{G}\left(\mathbb{A}_{\mathrm{f}}\right)$, alors le fibré induit sur la composante connexe $\Gamma_{g_{K}} \backslash X$ est $\Gamma_{g_{K}} \backslash X \times L$. L'image réciproque de ce dernier sur $X$ est le fibré trivial $X \times L$ car, $K$ étant assez petit au sens de (TF), $\Gamma_{g_{K}}$ agit librement sur $X$. 
Les compatibilités sont données par le diagramme commutatif suivant :

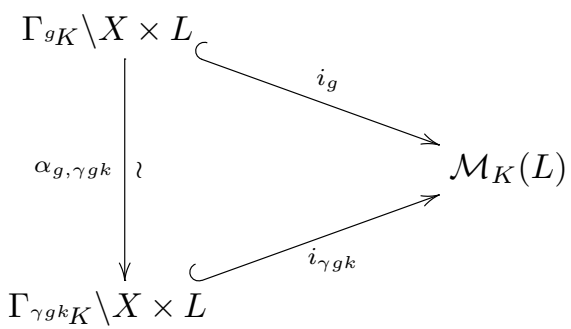

où pour $g \in \mathbf{G}\left(\mathbb{A}_{\mathrm{f}}\right), \gamma \in \mathbf{G}(\mathbb{Q}), k \in K, x \in X$ et $l \in L$,

$$
\begin{aligned}
i_{g}\left(\Gamma_{g_{K}}(x, l)\right) & =\mathbf{G}(\mathbb{Q})(x, g * l) K \quad \text { et } \\
\alpha_{g, \gamma g k}\left(\Gamma_{g_{K}}(x, l)\right) & =\Gamma_{\gamma k_{K}}\left(\gamma x, k^{-1} . l\right) .
\end{aligned}
$$

Le système local associé au $\mathbb{Z}[K]$-module $L$ est le faisceau (que nous noterons aussi $L$ ), sur l'espace topologique $\mathcal{M}_{K}$, des germes de sections localement constantes du fibré $\mathcal{M}_{K}(L)$.

Par comparaison de la cohomologie d'un groupe $\Gamma$ avec celle d'un espace $K(\Gamma, 1)$, la cohomologie d'un système local sur $\mathcal{M}_{K}$ se décompose sous la forme :

$$
\mathrm{H}^{\cdot}\left(\mathcal{M}_{K}, L\right)=\prod_{\mathbf{G}(\mathbb{Q}) \backslash \mathbf{G}\left(\mathbb{A}_{\mathbf{f}}\right) / K} \mathrm{H}^{\cdot}\left(\Gamma_{g_{K}}, L\right) .
$$

Proposition 4.3.1. - Soit L un faisceau sur $\partial \overline{\mathcal{M}}_{K}$.

Il existe une suite spectrale

$$
E_{1}^{i, j}=\prod_{c \subset \mathfrak{P}_{i+1}} \mathrm{H}^{j}\left(\partial_{c} \overline{\mathcal{M}}_{K}, L\right) \Rightarrow \mathrm{H}^{i+j}\left(\partial \overline{\mathcal{M}}_{K}, L\right)
$$

le produit de gauche étant sur les classes de $\mathbf{G}(\mathbb{Q})$-conjugaison $c \subset \mathfrak{P}_{i+1}$.

Démonstration. - On utilise le recouvrement fermé fini $\left(\partial_{c_{1}} \overline{\mathcal{M}}_{K}\right)_{c_{1} \subset \mathfrak{P}_{1}}$ du bord $\partial \overline{\mathcal{M}}_{K}$ décrit en 4.2.5. Rappelons que $c_{1}$ parcourt l'ensemble des classes de $\mathbf{G}(\mathbb{Q})$-conjugaison de $\mathbb{Q}$-sousgroupes paraboliques propres maximaux de G. La suite spectrale de Leray [16, II, th. 5.2.4] associée à ce recouvrement fermé est telle que

$$
E_{1}^{i, j}=\prod_{\substack{S \subset \mathfrak{P}_{1} \\ \# c(S)=i+1}} \mathrm{H}^{j}\left(\bigcap_{c_{1} \subset S} \partial_{c_{1}} \overline{\mathcal{M}}_{K}, L\right) \Rightarrow \mathrm{H}^{i+j}\left(\partial \overline{\mathcal{M}}_{K}, L\right)
$$

où le produit porte sur les sous-ensembles $S \subset \mathfrak{P}_{1}$ union d'exactement $i+1$ classes de conjugaison. Le lemme 4.2.1 permet de décomposer

$$
\mathrm{H}^{j}\left(\bigcap_{c_{1} \subset S} \partial_{c_{1}} \overline{\mathcal{M}}_{K}, L\right)=\prod_{s(c)=S} \mathrm{H}^{j}\left(\partial_{c} \overline{\mathcal{M}}_{K}, L\right) .
$$

D'où $E_{1}^{i, j}=\prod_{c \subset \mathfrak{P}_{i+1}} \mathrm{H}^{j}\left(\partial_{c} \overline{\mathcal{M}}_{K}, L\right)$. 


\subsubsection{Opérateurs de Hecke}

Nous renvoyons à la section 1 pour les notations et définitions des paires, opérateurs et anneaux de Hecke abstraits. Nous convenons de faire agir les groupes et monoïdes à gauche, et les anneaux de Hecke à droite. Si bien que, dans ce qui suit, partant d'une action (à gauche) de $D^{-1} \supset K$, nous obtenons une action (à droite) de $\mathcal{H}(K, D)$ (sur les $K$-invariants par exemple).

Soient $K \in \mathcal{T}$ et $D \subset \mathbf{G}\left(\mathbb{A}_{\mathrm{f}}\right)$ un sous-monoïde contenant $K$. Si $\xi \in D$, posons ${ }_{\xi} K:=K \cap{ }^{\xi} K$ et $K_{\xi}:=K \cap K^{\xi}$.

Le commensurateur d'un sous-groupe ouvert compact de $\mathbf{G}\left(\mathbb{A}_{\mathrm{f}}\right)$ est égal à $\mathbf{G}\left(\mathbb{A}_{\mathrm{f}}\right)$. L'anneau de Hecke $\mathcal{H}(K, D)$ agit naturellement sur la cohomologie de la variété modulaire $\mathcal{M}_{K}$. Plus précisément, si $L$ est un $\mathbb{Z}\left[D^{-1}\right]$-module, l'opérateur $K \xi K$ est défini à partir de la correspondance algébrique

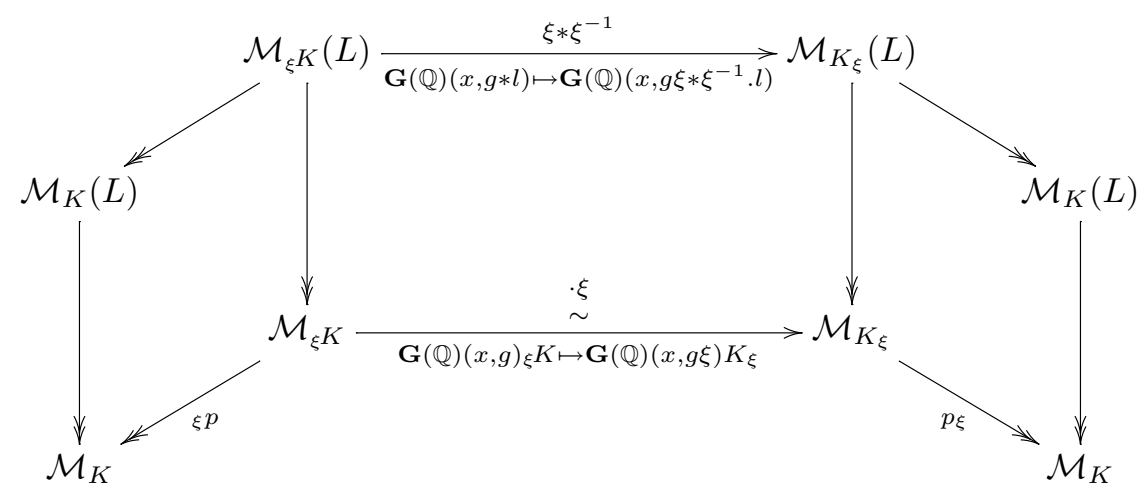

dans laquelle les carrés des deux côtés sont cartésiens. Ainsi le diagramme

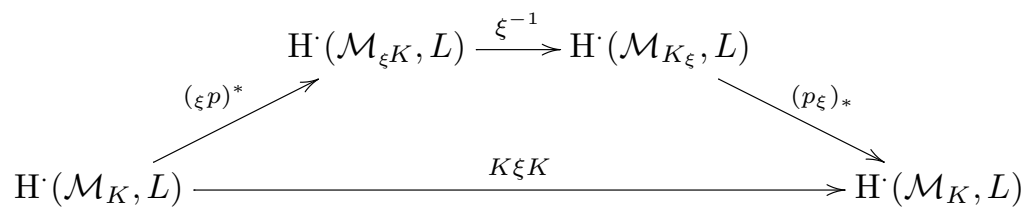

où $\left({ }_{\xi} p\right)^{*}$ et $\left(p_{\xi}\right)_{*}$ sont respectivement l'image inverse $\operatorname{par}_{\xi} p$ et la trace de $p_{\xi}$, est commutatif.

\subsubsection{Comparaison avec la cohomologie des groupes}

Afin de donner l'analogue adélique du résultat de comparaison de la cohomologie du groupe $\Gamma_{K}$ avec celle de l'espace $\Gamma_{K} \backslash X$, nous munissons, pour tout $\mathbb{Z}\left[D^{-1}\right]$-module $L$, le module induit ind ${ }^{\mathbf{G}\left(\mathbb{A}_{\mathrm{f}}\right)} L$ de deux actions qui commutent (nous identifions ce module induit à l'espace des fonctions sur $\mathbf{G}\left(\mathbb{A}_{\mathrm{f}}\right)$ à valeurs dans $\left.L\right)$ :

- l'action de $\mathbf{G}(\mathbb{Q})$ obtenue par restriction de l'action canonique du groupe $\mathbf{G}\left(\mathbb{A}_{\mathrm{f}}\right)$ sur le module induit : $(\gamma s)(g):=s\left(\gamma^{-1} g\right)$ quels que soient $\gamma \in \mathbf{G}(\mathbb{Q}), s \in$ ind $^{\mathbf{G}\left(\mathbb{A}_{\mathrm{f}}\right)} L$ et $g \in \mathbf{G}\left(\mathbb{A}_{\mathrm{f}}\right)$;

- et celle de $D^{-1}$ définie $\operatorname{par}\left(\xi^{-1} s\right)(g):=\xi^{-1} . s\left(g \xi^{-1}\right)$, pour tout $\xi \in D, s \in$ ind $^{\mathbf{G}\left(\mathbb{A}_{\mathrm{f}}\right)} L$ et tout $g \in \mathbf{G}\left(\mathbb{A}_{\mathrm{f}}\right)$.

En prenant les invariants sous chacune de ces actions nous obtenons :

- d'une part, l'espace

$$
\mathcal{F}(L):=\mathcal{F}\left(\mathbf{G}(\mathbb{Q}) \backslash \mathbf{G}\left(\mathbb{A}_{\mathrm{f}}\right), L\right)
$$


des fonctions sur $\mathbf{G}(\mathbb{Q}) \backslash \mathbf{G}\left(\mathbb{A}_{\mathrm{f}}\right)$ à valeurs dans $L$. C'est un sous- $D^{-1}$-module de ind ${ }^{\mathbf{G}\left(\mathbb{A}_{\mathrm{f}}\right)} L$, si bien que les groupes de cohomologie $\mathrm{H}^{\cdot}(K, \mathcal{F}(L))$ sont des $\mathcal{H}(K, D)$-modules;

- et de l'autre, le module induit

$$
\operatorname{ind}_{K}^{\mathbf{G}\left(\mathbb{A}_{\mathrm{f}}\right)} L=\mathrm{H}^{0}\left(K, \operatorname{ind}^{\mathbf{G}\left(\mathbb{A}_{\mathrm{f}}\right)} L\right) .
$$

C'est un sous- $\mathbf{G}(\mathbb{Q})$-module de ind $^{\mathbf{G}\left(\mathbb{A}_{\mathrm{f}}\right)} L$, muni naturellement d'une action de l'anneau de Hecke $\mathcal{H}(K, D)$ :

$$
s \mid K \xi K:=\sum_{K \xi \alpha \in K \xi K}(\xi \alpha)^{-1} . s
$$

pour tout $\xi \in D$ et tout $s \in \operatorname{ind}_{K}^{\mathbf{G}\left(\mathbb{A}_{\mathrm{f}}\right)} L$. Cette action commute avec celle de $\mathbf{G}(\mathbb{Q})$, si bien que les groupes de cohomologie

$$
\mathrm{H}^{\cdot}\left(\mathbf{G}(\mathbb{Q}), \operatorname{ind}_{K}^{\mathbf{G}\left(\mathbb{A}_{\mathrm{f}}\right)} L\right)
$$

sont aussi munis d'une action de $\mathcal{H}(K, D)$.

Proposition 4.3.2. - Soient $K \in \mathcal{T}, D \subset \mathbf{G}\left(\mathbb{A}_{\mathrm{f}}\right)$ un sous-monö̈de contenant $K$ et $L$ un $\mathbb{Z}\left[D^{-1}\right]$-module (à gauche).

Nous avons les isomorphismes canoniques de $\mathcal{H}(K, D)$-modules (à droite) suivants :

$$
\mathrm{H}^{\cdot}(K, \mathcal{F}(L)) \stackrel{\sim}{\leftarrow} \mathrm{H}^{\cdot}\left(\mathcal{M}_{K}, L\right) \stackrel{\sim}{\rightarrow} \mathrm{H}^{\cdot}\left(\mathbf{G}(\mathbb{Q}), \operatorname{ind}_{K}^{\mathbf{G}\left(\mathbb{A}_{\mathrm{f}}\right)} L\right) .
$$

Démonstration. - Tout d'abord, en degré 0, selon les compatibilités détaillées en 4.3.2, le groupe des sections globales du système local associé à $L$ s'identifie au groupe des fonctions $s \in \mathcal{F}(L)$ telles que pour tout $g \in \mathbf{G}\left(\mathbb{A}_{\mathrm{f}}\right)$ et tout $k \in K$,

$$
s(\mathbf{G}(\mathbb{Q}) g) \in L^{\Gamma g_{K}} \quad \text { et } \quad s(\mathbf{G}(\mathbb{Q}) g k)=k^{-1} \cdot s(\mathbf{G}(\mathbb{Q}) g) .
$$

Mais la première relation est une conséquence de la seconde car, pour tout $\gamma \in \Gamma_{g_{K}}$ :

$$
\gamma^{g} . s(\mathbf{G}(\mathbb{Q}) g)=s\left(\mathbf{G}(\mathbb{Q}) g\left(\gamma^{-1}\right)^{g}\right)=s\left(\mathbf{G}(\mathbb{Q}) \gamma^{-1} g\right)=s(\mathbf{G}(\mathbb{Q}) g) .
$$

Ainsi $\mathrm{H}^{0}(K, \mathcal{F}(L))=\mathrm{H}^{0}\left(\mathcal{M}_{K}, L\right)=\mathrm{H}^{0}\left(\mathbf{G}(\mathbb{Q}), \operatorname{ind}_{K}^{\mathbf{G}\left(\mathbb{A}_{\mathrm{f}}\right)} L\right)$.

En degré quelconque, les isomorphismes s'obtiennent par décalage car

- le foncteur qui, à $L$, associe le système local correspondant sur $\mathcal{M}_{K}$ est un foncteur exact de la catégorie des $D^{-1}$-modules dans celle des faisceaux sur $\mathcal{M}_{K}$, qui transforme les modules injectifs en faisceaux acycliques pour $\mathrm{H}^{\cdot}\left(\mathcal{M}_{K}, \cdot\right)$. Cette dernière propriété se déduit du résultat de comparaison sur chaque composante connexe :

$$
\mathrm{H}^{\cdot}\left(\Gamma_{g_{K}} \backslash X, L\right) \simeq \mathrm{H}^{\cdot}\left(\Gamma_{g_{K}}, L\right)
$$

- $\mathcal{F}$ est un foncteur exact de la catégorie des $D^{-1}$-modules dans elle-même qui conserve les objets injectifs. L'exactitude est immédiate et la conservation des injectifs vient de

$$
\operatorname{Hom}_{D}(\cdot, \mathcal{F}(L))=\operatorname{Hom}_{D}\left(\cdot \otimes_{\mathbb{Z}} \mathbb{Z}\left[\mathbf{G}(\mathbb{Q}) \backslash \mathbf{G}\left(\mathbb{A}_{\mathrm{f}}\right)\right], L\right)
$$


- ind $\mathrm{G}_{K}^{\mathrm{G}\left(\mathbb{A}_{\mathrm{f}}\right)}$ est un foncteur exact de la catégorie des $D^{-1}$-modules dans celle des $(\mathbf{G}(\mathbb{Q}), \mathcal{H}(K, D))$-bimodules, et transforme les modules injectifs en $\mathbf{G}(\mathbb{Q})$-modules injectifs. La conservation des injectifs résulte de

$$
\operatorname{Hom}_{\mathbf{G}(\mathbb{Q})}\left(\cdot, \operatorname{ind}_{K}^{\mathbf{G}\left(\mathbb{A}_{\mathrm{f}}\right)} L\right)=\operatorname{Hom}_{K}\left(\cdot \otimes_{\mathbb{Z}[\mathbf{G}(\mathbb{Q})]} \mathbb{Z}\left[\mathbf{G}\left(\mathbb{A}_{\mathrm{f}}\right)\right], L\right)
$$

et du fait que $\mathbb{Z}\left[\mathbf{G}\left(\mathbb{A}_{\mathrm{f}}\right)\right]$ est un $\mathbb{Z}[\mathbf{G}(\mathbb{Q})]$-module libre.

\subsubsection{Descente à $\mathcal{M}_{K}^{\mathrm{ad}}$}

Nous comparons ici la cohomologie des systèmes locaux sur

$$
\mathcal{M}_{K}^{\mathrm{ad}}:=\mathbf{G}(\mathbb{Q}) \backslash X^{\mathrm{ad}} \times \mathbf{G}\left(\mathbb{A}_{\mathrm{f}}\right) / K
$$

d'une part et sur $\mathcal{M}_{K}$ d'autre part.

On note $r_{\mathbf{R G}}=\operatorname{dim} \mathbf{R}_{\mathbb{R}} \mathbf{G}-\operatorname{dim} \mathbf{R}_{\mathbb{Q}} \mathbf{G}$ la différence entre le rang réel et le rang rationnel du radical de $\mathbf{G}$. Selon le théorème de Dirichlet pour les tores [25, cor. 1 du th. 4.14], $r_{\mathbf{R G}}$ est aussi le rang du groupe abélien libre $\Gamma_{K} \cap \mathbf{R}_{\mathbb{R}} \mathbf{G}(\mathbb{R})^{+}$lorsque $K \in \mathcal{T}$.

Corollaire 4.3.1. - Soient $K \in \mathcal{T}$ et $L$ un $\mathbb{Z}[K]$-module dont la restriction à $\Gamma_{K} \cap$ $\mathbf{R}_{\mathbb{R}} \mathbf{G}(\mathbb{R})^{+}$est triviale.

On a canoniquement $\mathrm{H}^{0}\left(\mathcal{M}_{K}^{\mathrm{ad}}, L\right) \stackrel{\sim}{\rightarrow} \mathrm{H}^{0}\left(\mathcal{M}_{K}, L\right)$ et il existe une suite exacte longue

$$
\begin{aligned}
& 0 \rightarrow \mathrm{H}^{1}\left(\mathcal{M}_{K}^{\mathrm{ad}}, L\right) \rightarrow \mathrm{H}^{1}\left(\mathcal{M}_{K}, L\right) \rightarrow \mathrm{H}^{0}\left(\mathcal{M}_{K}^{\mathrm{ad}}, L\right)^{r_{\mathrm{RG}}} \rightarrow \cdots \\
& \cdots \rightarrow \mathrm{H}^{i}\left(\mathcal{M}_{K}^{\mathrm{ad}}, L\right) \rightarrow \mathrm{H}^{i}\left(\mathcal{M}_{K}, L\right) \rightarrow \mathrm{H}^{i-1}\left(\mathcal{M}_{K}^{\mathrm{ad}}, L\right)^{r_{\mathbf{R G}}} \rightarrow \cdots .
\end{aligned}
$$

Démonstration. - L'analogue de l'isomorphisme $\mathrm{H}^{\cdot}\left(\mathcal{M}_{K}, L\right) \simeq \mathrm{H}^{\cdot}(K, \mathcal{F}(L))$ pour $\mathcal{M}_{K}^{\text {ad }}$ est $\mathrm{H}^{\cdot}\left(\mathcal{M}_{K}^{\text {ad }}, L\right) \simeq \mathrm{H}^{\cdot}\left(K^{\text {ad }}, \mathcal{F}(L)\right)$ où $K^{\text {ad }}:=K /\left(\Gamma_{K} \cap \mathbf{R}_{\mathbb{R}} \mathbf{G}(\mathbb{R})^{+}\right)$car ses composantes connexes sont des espaces $K\left(\Gamma_{g_{K}} /\left(\Gamma_{K} \cap \mathbf{R}_{\mathbb{R}} \mathbf{G}(\mathbb{R})^{+}\right), 1\right)$. De plus $\mathcal{F}(L)$ est un $\Gamma_{K} \cap \mathbf{R}_{\mathbb{R}} \mathbf{G}(\mathbb{R})^{+}$-module trivial dont les seuls groupes de cohomologie non triviaux sont

$$
\mathrm{H}^{0}\left(\Gamma_{K} \cap \mathbf{R}_{\mathbb{R}} \mathbf{G}(\mathbb{R})^{+}, \mathcal{F}(L)\right)=\mathcal{F}(L) \quad \text { et } \quad \mathrm{H}^{1}\left(\Gamma_{K} \cap \mathbf{R}_{\mathbb{R}} \mathbf{G}(\mathbb{R})^{+}, \mathcal{F}(L)\right)=\mathcal{F}(L)^{r_{\mathbf{R G}}}
$$

car $\Gamma_{K} \cap \mathbf{R}_{\mathbb{R}} \mathbf{G}(\mathbb{R})^{+}=\mathbb{Z}^{r_{\mathbf{R G}}}$. On obtient donc une suite spectrale de Hochschild-Serre sous la forme

$$
E_{2}^{i, j}=\mathrm{H}^{i}\left(\mathcal{M}_{K}^{\mathrm{ad}}, \mathrm{H}^{j}\left(\Gamma_{K} \cap \mathbf{R}_{\mathbb{R}} \mathbf{G}(\mathbb{R})^{+}, L\right)\right) \quad \Rightarrow \quad \mathrm{H}^{i+j}\left(\mathcal{M}_{K}, L\right)
$$

qui dégénère et donne la suite exacte du corollaire.

\subsubsection{Changement de niveau}

Nous donnons l'analogue du lemme de Shapiro et de la suite spectrale de Hochschild-Serre :

Corollaire 4.3.2. - Soient $K^{\prime} \in \mathcal{T}$ et $K \in \mathcal{T}$ deux sous-groupes tels que $K^{\prime} \subset K$ et L un $\mathbb{Z}\left[K^{\prime}\right]$-module.

Les morphismes canoniques:

$$
\mathrm{H}^{\cdot}\left(\mathcal{M}_{K}, \operatorname{ind}_{K^{\prime}}^{K} L\right) \stackrel{\sim}{\rightarrow} \mathrm{H}^{\cdot}\left(\mathcal{M}_{K^{\prime}}, L\right)
$$

sont des isomorphismes.

Démonstration. - C'est une conséquence immédiate de la proposition précédente puisque $\operatorname{ind}_{K}^{\mathbf{G}\left(\mathbb{A}_{\mathrm{f}}\right)} \operatorname{ind}_{K^{\prime}}^{K} L=\operatorname{ind}_{K^{\prime}}^{\mathbf{G}\left(\mathbb{A}_{\mathrm{f}}\right)} L$. 
Corollaire 4.3.3. - Soient $K \in \mathcal{T}, D \subset \mathbf{G}\left(\mathbb{A}_{\mathrm{f}}\right)$ un sous-monoïde contenant $K$ et $L$ un $\mathbb{Z}\left[D^{-1}\right]$-module (à gauche).

Pour tout sous-groupe $K^{\prime} \in \mathcal{T}$ distingué, au sens du paragraphe 1.2, dans la paire $(K, D)$, nous avons une suite spectrale:

$$
E_{2}^{i, j}=\mathrm{H}^{i}\left(K / K^{\prime}, \mathrm{H}^{j}\left(\mathcal{M}_{K^{\prime}}, L\right)\right) \quad \Rightarrow \quad \mathrm{H}^{i+j}\left(\mathcal{M}_{K}, L\right)
$$

Hecke-équivariante (i.e. $\mathcal{H}(K, D)$-équivariante).

Démonstration. - Ici encore, une conséquence immédiate de la proposition précédente, et de la suite spectrale de Hochschild-Serre Hecke-équivariante du corollaire 1.2.1.

\subsubsection{Torsion par un caractère}

Soient $K \in \mathcal{T}, D \subset \mathbf{G}\left(\mathbb{A}_{\mathrm{f}}\right)$ un sous-monoïde contenant $K$ et $\chi: D \rightarrow A^{\times}$un morphisme de monoïdes, trivial sur $K$, à valeurs dans le groupe des éléments inversibles d'un anneau commutatif $A$.

Pour tout $A\left[D^{-1}\right]$-module à gauche $M$ et tout $A \otimes_{\mathbb{Z}} \mathcal{H}(K, D)$-module à droite $N$, nous notons

- $M(\chi)$ le $A\left[D^{-1}\right]$-module à gauche $M$ tordu par $\chi: \xi^{-1} \cdot \chi^{m}=\chi(\xi)^{-1} \xi^{-1} \cdot m$ pour tout $m \in M$ et tout $\xi \in D$;

- $N(\chi)$ le $A \otimes_{\mathbb{Z}} \mathcal{H}(K, D)$-module à droite $N$ tordu par $\chi:\left.n\right|_{\chi} K \xi K:=\chi(\xi)^{-1} n \mid K \xi K$ pour tout $n \in N$ et tout $\xi \in D$.

Lemme 4.3.2. - Soient $K \in \mathcal{T}, D \subset \mathbf{G}\left(\mathbb{A}_{\mathrm{f}}\right)$ un sous-monö̈de contenant $K, A$ un anneau commutatif et $L$ un $A\left[D^{-1}\right]$-module (à gauche).

Pour tout morphisme de monö̈des $\chi: D \rightarrow A^{\times}$trivial sur $K$, nous avons un isomorphisme canonique de $A \otimes_{\mathbb{Z}} \mathcal{H}(K, D)$-modules (à droite) :

$$
\mathrm{H}^{\cdot}\left(\mathcal{M}_{K}, L(\chi)\right) \stackrel{\sim}{\rightarrow} \mathrm{H}^{\cdot}\left(\mathcal{M}_{K}, L\right)(\chi) .
$$

Démonstration. - En tant que $A$-modules, les deux modules à comparer sont égaux puisque $\chi$ est trivial sur $K$. Il reste à montrer que l'action des opérateurs de Hecke est la même sur ces modules. Cela résulte de $\mathcal{F}(L(\chi))=\mathcal{F}(L)(\chi)$ puis du calcul sur les cochaînes.

\subsubsection{Réseaux}

Le lemme suivant, dont la preuve est standard, sera par exemple appliqué à la partie quasiordinaire $e_{\mathrm{qo}} \mathrm{H}^{\cdot}\left(\mathcal{M}_{K}, \cdot\right)$ de la cohomologie

LEMmE 4.3.3. - Soient $\mathcal{O}$ un anneau commutatif intègre de corps des fractions $\mathcal{K}, K$ un groupe et $L$ un $\mathcal{O}[K]$-module sans $\mathcal{O}$-torsion.

$\mathrm{Si} \mathrm{H}^{\cdot}$ est un foncteur cohomologique, de la catégorie des $\mathcal{O}[K]$-modules dans celle des $\mathcal{O}$-modules, qui commute aux limites inductives, alors, pour tout $i \in \mathbb{N}$, nous avons la suite exacte courte de $\mathcal{O}$-modules :

$$
0 \rightarrow \mathrm{H}^{i}(L) \otimes_{\mathcal{O}} \mathcal{K} / \mathcal{O} \rightarrow \mathrm{H}^{i}\left(L \otimes_{\mathcal{O}} \mathcal{K} / \mathcal{O}\right) \rightarrow \mathrm{H}^{i+1}(L)_{\text {tor }} \rightarrow 0 .
$$

Si, de plus, $\mathrm{H}^{i+1}(L)$ est de type fini sur $\mathcal{O}$ alors

- $\mathrm{H}^{i}\left(L \otimes_{\mathcal{O}} \mathcal{K} / \mathcal{O}\right)$ est fini si et seulement si $\mathrm{H}^{i}\left(L \otimes_{\mathcal{O}} \mathcal{K}\right)=0$

- $\mathrm{H}^{i}\left(L \otimes_{\mathcal{O}} \mathcal{K} / \mathcal{O}\right)$ est divisible sur $\mathcal{O}$ si et seulement si $\mathrm{H}^{i+1}(L)$ n'a pas de torsion sur $\mathcal{O}$;

- $\mathrm{H}^{i}\left(L \otimes_{\mathcal{O}} \mathcal{K} / \mathcal{O}\right)=0$ si et seulement si $\mathrm{H}^{i}\left(L \otimes_{\mathcal{O}} \mathcal{K}\right)=0$ et $\mathrm{H}^{i+1}(L)$ n'a pas de torsion $\operatorname{sur} \mathcal{O}$. 


\section{Cohomologie quasi-ordinaire}

Maintenant, $\mathbf{G}$ est un groupe réductif connexe sur $\mathbb{Q}$ et nous fixons un nombre premier $p$.

Dans cette section, nous utilisons les algèbres de Hecke paraboliques en $p$ de la section 3 et la comparaison de la proposition 4.3.2 entre la cohomologie de la variété $\mathcal{M}_{K}$ et celle du groupe de niveau $K$ pour contrôler la cohomologie de niveau fini.

Plus précisément, nous commençons par établir une suite spectrale liant, au moyen des opérateurs diamants, la cohomologie de niveau de type $\Gamma_{0}\left(p^{\infty}\right)$ à celle de type $\Gamma_{1}\left(p^{\infty}\right)$ (voir th. 5.2.1). Nous construisons au paragraphe 5.3.2 l'idempotent $\mathbf{P}$-quasi-ordinaire $e_{\mathrm{qo}}$ qui découpe la partie de la cohomologie sur laquelle $\Lambda_{\mathrm{M}}^{+} \simeq \mathbb{N} T_{1} \oplus \cdots \oplus \mathbb{N} T_{r}$ agit par automorphismes. Les relations entre opérateurs de Hecke du lemme 3.3.1, résultant des propriétés de contraction montrent que la cohomologie $\mathbf{P}$-quasi-ordinaire de niveau de type $\Gamma_{0}\left(p^{n}\right)$ est indépendante du niveau $n$ (lemme 5.3.2). Il en résulte une suite spectrale ( $c f$. cor. 5.3.2) liant la cohomologie $\mathbf{P}$-quasiordinaire de niveau fini de type $\Gamma_{0}\left(p^{n}\right)$ à celle de niveau infini de type $\Gamma_{1}\left(p^{\infty}\right)$.

En application de ce dernier résultat, nous donnons deux théorèmes comparant la cohomologie $\mathbf{P}$-quasi-ordinaire de niveau de type $\Gamma_{0}\left(p^{n}\right)$ à celle de type $\Gamma_{1}\left(p^{\infty}\right)$ (th. 5.4.1 et 5.4.2). Le premier donne des conditions suffisantes pour avoir un isomorphisme alors que le second, sous des hypothèses plus faibles, donne un morphisme à noyau et conoyau finis.

Hida a montré ces résultats dans le cas du groupe linéaire $\mathbf{G}=\mathbf{G L}(n)$ dans [19], où il les appelle théorèmes de contrôle fort et faible.

Notre méthode diffère de [19] par l'utilisation de la suite spectrale du corollaire 5.3.2. C'est grâce au point de vue adélique que nous avons pu faire apparaître les opérateurs diamants en $p$ dans une telle suite spectrale.

Dans la prochaine section, des théorèmes de contrôle, nous déduirons certaines propriétés de l'algèbre de Hecke $\mathbf{P}$-quasi-ordinaire universelle.

\subsection{Sous-groupes de niveau}

\subsubsection{Donnée en $p$}

Nous reprenons les notations suivantes des sections 2 et 3 appliquées au groupe $\mathbf{G}$ sur le corps $p$-adique $\mathbb{Q}_{p}$ :

- les $\mathbb{Q}_{p}$-groupes paraboliques opposés $\mathbf{P} \simeq \mathbf{M} \ltimes \mathbf{U}$ et $\mathbf{P}^{-} \simeq \mathbf{M} \ltimes \mathbf{U}^{-}$du paragraphe 2.3 ;

- la partie bornée et bien placée $\Omega$ de l'immeuble de Bruhat-Tits de $\mathbf{G}$ sur $\mathbb{Q}_{p}$ de 2.4;

- les $\mathbb{Z}_{p}$-schémas en groupes $\mathbf{P}_{\Omega}=\mathbf{M}_{\Omega} \ltimes \mathbf{U}_{\Omega}$ et $\mathbf{P}_{\Omega}^{-}=\mathbf{M}_{\Omega} \ltimes \mathbf{U}_{\Omega}^{-}$de 2.4;

- les sous-monoïdes $\mathbf{R M}\left(\mathbb{Q}_{p}\right)^{+} \subset \mathbf{R M}\left(\mathbb{Q}_{p}\right), \Lambda_{\mathbf{M}}^{+} \subset \Lambda_{\mathbf{M}}$ de 2.4;

- l'ensemble $\mathcal{T}_{\text {bp }}$ de 2.4 ;

- les sous-groupes $\mathbf{P}_{\Omega}\left(\mathbb{Z}_{p}\right)^{\prime}=\mathbf{M}_{\Omega}\left(\mathbb{Z}_{p}\right)^{\prime} \ltimes \mathbf{U}_{\Omega}\left(\mathbb{Z}_{p}\right)$ de 3 ;

- le morphisme $\sigma: \mathbf{R M}\left(\mathbb{Q}_{p}\right) \rightarrow \Lambda_{\mathbf{M}}$ de 3.1 ;

- le groupe profini

$$
C:=\mathbf{M}_{\Omega}\left(\mathbb{Z}_{p}\right) / \mathbf{M}_{\Omega}\left(\mathbb{Z}_{p}\right)^{\prime}=\varliminf_{U \in \mathcal{T}_{\mathrm{bp}}} C_{U}
$$

et les sous-monoïdes $D_{1, p} \subset D_{0, p}$ de 3.1 .

\subsubsection{Donnée auxiliaire}

On note $\mathbb{A}_{\mathrm{f}}^{(S)}$ l'algèbre des adèles finis, hors d'un ensemble fini $S$ de nombres premiers, du corps $\mathbb{Q}$. On note simplement $\mathbb{A}_{\mathrm{f}}^{(p)}:=\mathbb{A}_{\mathrm{f}}^{(\{p\})}$.

Nous fixons aussi

- un sous-groupe ouvert compact $K^{(p)}$ de $\mathbf{G}\left(\mathbb{A}_{\mathrm{f}}^{(p)}\right)$ tel que $\mathbf{G}_{\Omega}\left(\mathbb{Z}_{p}\right) K^{(p)}$ est un sous-groupe assez petit au sens de (TF), 
- un sous-monoïde $D^{(p)}$ de $\mathbf{G}\left(\mathbb{A}_{\mathrm{f}}^{(p)}\right)$ tel que l'anneau de Hecke

$$
\mathcal{H}^{(p)}:=\mathcal{H}\left(K^{(p)}, D^{(p)}\right)
$$

est commutatif.

D'après [10, I.4.4.9], ou [29], la dernière condition est remplie, par exemple, si la paire de Hecke $K^{(p)} \subset D^{(p)}$ est de la forme

$$
K^{(p)}=\tilde{K} \prod_{l \notin \tilde{S} \cup\{p\}} K_{l} \subset D^{(p)}=\tilde{K} \mathbf{G}\left(\mathbb{A}_{\mathrm{f}}^{(\tilde{S} \cup\{p\})}\right)
$$

où $\tilde{S}$ est un sous-ensemble fini de nombres premiers distincts de $p, l$ parcours l'ensemble des nombres premiers hors de $\tilde{S} \cup\{p\}, \tilde{K}$ est un sous-groupe ouvert compact de $\prod_{l \in \tilde{S}} \mathbf{G}\left(\mathbb{Q}_{l}\right)$ et pour tout $l \notin \tilde{S} \cup\{p\}, K_{l}$ est un sous-groupe spécial de $\mathbf{G}\left(\mathbb{Q}_{l}\right)$, c'est-à-dire le fixateur d'un point spécial de l'immeuble de Bruhat-Tits élargi (cf. [10, II.5.1.29]) de $\mathbf{G}_{\mathbb{Q}_{l}}$.

\subsubsection{Anneaux de Hecke adéliques}

Nous considérons les sous-groupes

$$
K_{1}:=K^{(p)} \mathbf{P}_{\Omega}\left(\mathbb{Z}_{p}\right)^{\prime} \subset K_{0}:=K^{(p)} \mathbf{P}_{\Omega}\left(\mathbb{Z}_{p}\right)
$$

et les sous-monoïdes

$$
D_{1}:=D^{(p)} D_{1, p} \subset D_{0}:=D^{(p)} D_{0, p} .
$$

On se reportera en 3.1 pour la définition des sous-monoïdes $D_{1, p}$ et $D_{0, p}$.

Par la suite, nous noterons souvent $K_{*} \subset D_{*}$ l'une des paires de Hecke $K_{1} \subset D_{1}, K_{1} \subset D_{0}$ ou $K_{0} \subset D_{0}$.

On identifie un sous-groupe $U \in \mathcal{T}_{\mathrm{bp}}$ à son image par l'injection canonique $\mathbf{G}\left(\mathbb{Q}_{p}\right) \subset \mathbf{G}\left(\mathbb{A}_{\mathrm{f}}\right)$.

D'après le paragraphe 1.3 , les isomorphismes $\varphi_{\xi p}$ de (18) donnent lieu à

- un système projectif $\mathcal{H}\left(U K_{*}, U D_{*}\right)$, indexé par $U \in \mathcal{T}_{\mathrm{bp}}$, d'anneaux de Hecke de niveau fini,

- et un morphisme $\mathcal{H}\left(K_{*}, D_{*}\right) \rightarrow\left(\mathcal{H}\left(U K_{*}, U D_{*}\right)\right)_{U \in \mathcal{T}_{\text {bp }}}$ de l'anneau de Hecke de niveau infini dans ce système projectif.

Selon les propositions 3.1.1 et 3.2.1, les anneaux de Hecke et les morphismes en jeu sont

- pour la paire $\left(K_{1}, D_{1}\right)$ : les applications identiques

$$
\mathcal{H}^{(p)}\left[\Lambda_{\mathbf{M}}^{+}\right] \rightarrow\left(\mathcal{H}^{(p)}\left[\Lambda_{\mathbf{M}}^{+}\right]\right)_{U \in \mathcal{T}_{\mathrm{bp}}}
$$

- pour la paire $\left(K_{1}, D_{0}\right)$ : les applications

$$
\mathcal{H}^{(p)}\left[\Lambda_{\mathbf{M}}^{+}\right][C] \rightarrow\left(\mathcal{H}^{(p)}\left[\Lambda_{\mathbf{M}}^{+}\right]\left[C_{U}\right]\right)_{U \in \mathcal{T}_{\mathrm{bp}}}
$$

induites par les morphismes quotients $C \rightarrow C_{U}$,

- pour la paire $\left(K_{0}, D_{0}\right)$ : à nouveau les applications identiques

$$
\mathcal{H}^{(p)}\left[\Lambda_{\mathbf{M}}^{+}\right] \rightarrow\left(\mathcal{H}^{(p)}\left[\Lambda_{\mathbf{M}}^{+}\right]\right)_{U \in \mathcal{T}_{\mathrm{bp}}}
$$

On munit les anneaux $\mathcal{H}^{(p)}\left[\Lambda_{\mathbf{M}}^{+}\right]$et $\mathcal{H}^{(p)}\left[\Lambda_{\mathbf{M}}^{+}\right]\left[C_{U}\right]$ de la topologie discrète, et l'anneau $\mathcal{H}^{(p)}\left[\Lambda_{\mathrm{M}}^{+}\right][C]$ de la topologie dont un système fondamental de voisinages de zéro est formé des noyaux des morphismes $\mathcal{H}^{(p)}\left[\Lambda_{\mathbf{M}}^{+}\right][C] \rightarrow \mathcal{H}^{(p)}\left[\Lambda_{\mathbf{M}}^{+}\right]\left[C_{U}\right]$. 
Ainsi, un module sur $\mathcal{H}^{(p)}\left[\Lambda_{\mathbf{M}}\right][C]$ est discret si et seulement si il est discret en tant que module sur le groupe profini $C$.

\subsection{Cohomologie de niveau infini}

\subsubsection{Variété modulaire de niveau infini}

On notera $\mathcal{M}_{K_{*}}$ le système projectif, indexé par $U \in \mathcal{T}_{\mathrm{bp}}$, formé des variétés modulaires $\mathcal{M}_{U K_{*}}$ de niveau $U K_{*}$.

Soit $U \in \mathcal{T}_{\mathrm{bp}}$. L'ensemble des sous-groupes de $U$ appartenant à $\mathcal{T}_{\mathrm{bp}}$ est un sous-ensemble cofinal de $\mathcal{T}_{\text {bp }}$.

Si $L$ est un $\mathbb{Z}\left[U K_{*}\right]$-module, on définit formellement la cohomologie du système projectif $\mathcal{M}_{K_{*}}$ à valeurs dans $L$ par

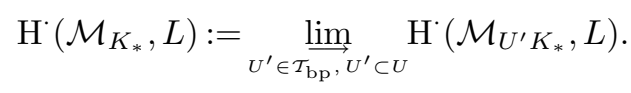

Rappelons que nous notons encore $L$ le système local défini (cf. 4.3.2) sur $\mathcal{M}_{U^{\prime} K_{*}}$.

Par exactitude du foncteur limite inductive et du fait que tout $U K_{*}$-module injectif est aussi un $U^{\prime} K_{*}$-module injectif (pour tout sous-groupe $U^{\prime} \in \mathcal{T}_{\mathrm{bp}}$ de $U$ ), les $\mathrm{H}^{\cdot}\left(\mathcal{M}_{K_{*}}, \cdot\right)$ sont les foncteurs dérivés à droite de $\mathrm{H}^{0}\left(\mathcal{M}_{K_{*}}, \cdot\right)$, de la catégorie des $U K_{*}$-modules dans celle des groupes abéliens.

\subsubsection{Comparaison avec la cohomologie des groupes}

Nous définissons les groupes de cohomologie

$$
\underline{\mathrm{H}}\left(K_{*}, \cdot\right):=\varliminf_{U^{\prime} \in \mathcal{T}_{\mathrm{bp}, U^{\prime}} \subset U} \mathrm{H}^{\cdot}\left(U^{\prime} K_{*}, \cdot\right)
$$

de la catégorie des $U K_{*}$-modules dans celle des groupes abéliens.

Par les mêmes arguments qu' au paragraphe précédent, ce sont les foncteurs dérivés à droite de $\underline{\mathrm{H}}^{0}\left(K_{*}, \cdot\right)$.

Proposition 5.2.1. - Soient $U \in \mathcal{T}_{\text {bp }}$ et $L$ un $\mathbb{Z}\left[U K_{*}\right]$-module.

On a un isomorphisme canonique :

$$
\mathrm{H}^{\cdot}\left(\mathcal{M}_{K_{*}}, L\right) \stackrel{\sim}{\rightarrow} \underline{\mathrm{H}} \cdot\left(K_{*}, \mathcal{F}(L)\right) .
$$

Démonstration. - C'est l'isomorphisme obtenu par passage à la limite à partir des isomorphismes $\mathrm{H}^{\cdot}\left(\mathcal{M}_{U K_{*}}, L\right) \simeq \mathrm{H}^{\cdot}\left(U K_{*}, \mathcal{F}(L)\right)$ de la proposition 4.3.2.

\subsubsection{Structure de $\mathcal{H}\left(K_{*}, D_{*}\right)$-module discret}

Soit $L$ un $\mathbb{Z}\left[\left(U D_{*}\right)^{-1}\right]$-module. Les applications $\varphi_{\xi p}$ de (18) étant bijectives, la proposition 1.3.1 munit les groupes $\underline{\mathrm{H}}\left(K_{*}, \cdot\right)$ d'une structure de $\mathcal{H}\left(K_{*}, D_{*}\right)$-module discret.

Selon la proposition précédente, il en est de même des groupes $\mathrm{H}^{\cdot}\left(\mathcal{M}_{K_{*}}, L\right)$. Par exemple, si $L$ est un $\mathbb{Z}\left[\left(U D_{0}\right)^{-1}\right]$-module, la remarque de la fin du paragraphe 5.1.3 permet de considérer les groupes $\mathrm{H} \cdot\left(\mathcal{M}_{K_{1}}, L\right)$ comme des $C$-modules discrets et de considérer les groupes de cohomologie continue

$$
\mathrm{H}^{\cdot}\left(C, \mathrm{H}^{\cdot}\left(\mathcal{M}_{K_{1}}, L\right)\right) .
$$

Les éléments de $C$ sont la généralisation des opérateurs "diamants" classiques, ou plutôt de leurs inverses puisque nous les avons traités comme des opérateurs de Hecke, lesquels agissent à droite. 


\subsubsection{Suite spectrale de changement de niveau}

THÉORÈME 5.2.1. - Soient $U \in \mathcal{T}_{\mathrm{bp}}$ et $L$ un $\mathbb{Z}\left[\left(U D_{0}\right)^{-1}\right]$-module.

On a une suite spectrale $\mathcal{H}^{(p)}\left[\Lambda_{\mathrm{M}}^{+}\right]$-équivariante :

$$
E_{2}^{i, j}=\mathrm{H}^{i}\left(C, \mathrm{H}^{j}\left(\mathcal{M}_{K_{1}}, L\right)\right) \quad \Rightarrow \quad \mathrm{H}^{i+j}\left(\mathcal{M}_{K_{0}}, L\right) .
$$

Démonstration. - D'après la proposition 5.2.1, il suffit de prouver la suite spectrale correspondante en remplaçant les cohomologies $\mathrm{H}^{\cdot}\left(\mathcal{M}_{K_{*}}, \cdot\right)$ par $\underline{\mathrm{H}}\left(K_{*}, \cdot\right)$.

On se place d'abord en degré zéro.

Selon la proposition 3.2.1, pour tout sous-groupe $U^{\prime} \in \mathcal{T}_{\mathrm{bp}}$ de $U$, le sous-groupe $U^{\prime} \mathbf{P}_{\Omega}\left(\mathbb{Z}_{p}\right)^{\prime}$ est distingué dans la paire $U^{\prime} \mathbf{P}_{\Omega}\left(\mathbb{Z}_{p}\right) \subset U^{\prime} D_{0}$. Comme dans le théorème 1.2.1, on en déduit que l'égalité

$$
\mathrm{H}^{0}\left(C_{U^{\prime}}, \mathrm{H}^{0}\left(U^{\prime} K_{1}, L\right)\right)=\mathrm{H}^{0}\left(U^{\prime} K_{0}, L\right)
$$

est équivariante sous $\mathcal{H}\left(U^{\prime} K_{0}, U^{\prime} D_{0}\right)=\mathcal{H}^{(p)}\left[\Lambda_{\mathbf{M}}^{+}\right]$. Par passage à la limite, on obtient l'égalité $\mathcal{H}^{(p)}\left[\Lambda_{\mathbf{M}}^{+}\right]$-équivariante

$$
\mathrm{H}^{0}\left(C, \underline{\mathrm{H}}^{0}\left(K_{1}, L\right)\right)=\underline{\mathrm{H}}^{0}\left(K_{0}, L\right) .
$$

Pour obtenir la suite spectrale, il suffit maintenant de montrer que le foncteur $\underline{\mathrm{H}}^{0}\left(K_{1}, \cdot\right)$ transforme tout $\left(U D_{0}\right)^{-1}$-module injectif $I$ en un module discret acyclique pour $\mathrm{H}^{\cdot}(C, \cdot)$.

Soit $I$ un tel module. Puisque $\mathbb{Z}\left[U D_{0}\right]$ est un module (à gauche) libre sur $\mathbb{Z}\left[U^{\prime} K_{0}\right]\left(U^{\prime} \in \mathcal{T}_{\mathrm{bp}}\right.$, $U^{\prime} \subset U$ ), les isomorphismes

$$
\begin{aligned}
\operatorname{Hom}_{C_{U^{\prime}}}\left(\cdot, \mathrm{H}^{0}\left(U^{\prime} K_{1}, I\right)\right) & \simeq \operatorname{Hom}_{C_{U^{\prime}}}\left(\cdot, \operatorname{Hom}_{U^{\prime} K_{0}}\left(\mathbb{Z}\left[C_{U^{\prime}}\right], I\right)\right) \\
& \simeq \operatorname{Hom}_{U^{\prime} K_{0}}(\cdot, I) \\
& \simeq \operatorname{Hom}_{\left(U D_{0}\right)^{-1}}\left(\cdot \otimes_{\mathbb{Z}\left[U^{\prime} K_{0}\right]} \mathbb{Z}\left[U D_{0}\right], I\right)
\end{aligned}
$$

montrent que $\mathrm{H}^{0}\left(U^{\prime} K_{1}, I\right)$ est un $C_{U^{\prime}}$-module injectif. D'où

$$
\mathrm{H}^{\cdot}\left(C, \underline{\mathrm{H}}^{0}\left(K_{1}, I\right)\right) \simeq \underbrace{}_{U^{\prime} \in \mathcal{T}_{\mathrm{bp}}, U^{\prime} \subset U} \mathrm{lim}^{\cdot}\left(C_{U^{\prime}}, \mathrm{H}^{0}\left(U^{\prime} K_{1}, I\right)\right)=0 .
$$

\subsection{Quasi-ordinarité}

Dans cette sous-section $\mathcal{O}$ est un anneau local, noethérien et hensélien. On note $\mathcal{H}_{\mathcal{O}}^{(p)}:=$ $\mathcal{H}^{(p)} \otimes_{\mathbb{Z}} \mathcal{O}$.

\subsubsection{Construction d'idempotents}

Le lemme d'algèbre commutative suivant permet de construire l'idempotent qui découpe la partie quasi-ordinaire dans la cohomologie de la variété modulaire.

LEMME 5.3.1. - Soient $R$ un anneau local hensélien et $\mathrm{h}$ une $R$-algèbre commutative.

On suppose h munie d'une topologie linéaire dont un système fondamental de voisinages de 0 est formé d'idéaux $I \subset \mathrm{h}$ tels que $\mathrm{h} / I$ est une algèbre finie sur $R$.

Alors l'algèbre séparée complétée $\widehat{\mathrm{h}}$ de $\mathrm{h}$ est un produit (éventuellement infini) d'anneaux locaux.

Remarquons qu'aucune hypothèse n'est faite quant à la compatibilité entre la topologie de h et celle de l'anneau local $R$. 
Démonstration. - Notons $\mathcal{T}$ un système fondamental de voisinages de 0 de $\mathrm{h}$ ayant la propriété de l'énoncé et $\mathcal{M}$ l'ensemble des idéaux maximaux de $\mathrm{h}$.

Puisque $R$ est local, toute algèbre finie $R^{\prime}$ sur $R$ est semi-locale [9, V, §2, prop. 1 et prop. 3]. De plus, d'après l'hypothèse hensélienne [26, I.1, prop. 3], l'algèbre $R^{\prime}$ se décompose sous la forme du produit des localisées en ses idéaux maximaux.

En particulier, pour tout $I \in \mathcal{T}$, l'algèbre $\mathrm{h} / I$ se décompose sous la forme

$$
\mathrm{h} / I \simeq \prod_{\mathfrak{m} \in \mathcal{M}} \mathrm{h}_{\mathfrak{m}} / I \mathrm{~h}_{\mathfrak{m}}
$$

où les composantes pour lesquelles $I \not \subset \mathfrak{m}$ sont triviales.

En passant à la limite projective sur $I \in \mathcal{T}$, on obtient

$$
\widehat{\mathrm{h}} \stackrel{\sim}{\rightarrow} \prod_{\mathfrak{m} \in \mathcal{M}} \widehat{\mathrm{h}_{\mathfrak{m}}} \quad \text { avec } \quad \widehat{\mathrm{h}_{\mathfrak{m}}}:=\lim _{\overparen{I} \in \overline{\mathcal{T}}} \mathrm{h}_{\mathfrak{m}} / I \mathrm{~h}_{\mathfrak{m}}=\lim _{I \in \overleftarrow{T}, I \subset \mathfrak{m}} \mathrm{h}_{\mathfrak{m}} / I \mathrm{~h}_{\mathfrak{m}}
$$

Si $\mathfrak{m}$ est un idéal ouvert alors l'anneau $\widehat{\mathrm{h}_{\mathfrak{m}}}$ est local car, si $x \notin \mathfrak{m} \widehat{\mathrm{h}_{\mathfrak{m}}}$, son image dans chaque $\mathrm{h}_{\mathfrak{m}} / I \mathrm{~h}_{\mathfrak{m}}(I \subset \mathfrak{m})$ est inversible, donc $x$ est inversible.

Si $\mathfrak{m}$ n'est pas ouvert, alors $\widehat{\mathrm{h}_{\mathfrak{m}}}=1$.

COROLlaire 5.3.1. - Sous les mêmes hypothèses que le lemme précédent, à toute partie $S$ de $\mathrm{h}$ est associé un unique idempotent $e \in \widehat{\mathrm{h}}$ tel que, dans la décomposition $\widehat{\mathrm{h}}=e \widehat{\mathrm{h}} \oplus(1-e) \widehat{\mathrm{h}}$, tous les élément de $e S:=\{e s\}_{s \in S}$ sont inversibles dans $e \widehat{\mathrm{h}}$ et il existe au moins un élément de $(1-e) S$ qui est topologiquement nilpotent dans $(1-e) \widehat{\mathrm{h}}$.

Démonstration. - Grâce au lemme précédent, il suffit de définir e comme étant l'idempotent dont les composantes sont nulles exactement sur les facteurs $\widehat{\mathrm{h}}_{\mathfrak{m}}$ tels que $\mathfrak{m}$ est un idéal maximal de $\widehat{\mathrm{h}}$ rencontrant l'image de $S$.

\subsubsection{L'idempotent quasi-ordinaire}

On munit la $\mathcal{O}$-algèbre $\mathcal{O}\left[\Lambda_{\mathrm{M}}^{+}\right]$du monoïde $\Lambda_{\mathrm{M}}^{+}$de la topologie dont un système fondamental de voisinages de 0 est formé de tous les idéaux $I \subset \mathcal{O}\left[\Lambda_{\mathbf{M}}^{+}\right]$tels que $\mathcal{O}\left[\underline{\Lambda_{\mathbf{M}}^{+}}\right] / I$ est une $\mathcal{O}$-algèbre finie. Ce système forme bien une base de filtre car $\mathcal{O}$ est noethérien. Soit $\overline{\mathcal{O}\left[\Lambda_{\mathbf{M}}^{+}\right]}$l'algèbre séparée complétée de $\mathcal{O}\left[\Lambda_{\mathbf{M}}^{+}\right]$pour cette topologie, et $e_{\mathrm{qo}} \in \overline{\mathcal{O}\left[\Lambda_{\mathbf{M}}^{+}\right]}$l'idempotent quasi-ordinaire associé, grâce au corollaire 5.3.1, à la partie $\Lambda_{\mathrm{M}}^{+} \subset \mathcal{O}\left[\Lambda_{\mathrm{M}}^{+}\right]$.

La partie quasi-ordinaire d'un $\overline{\mathcal{O}\left[\Lambda_{\mathrm{M}}^{+}\right]}$-module $M$ est le projeté $e_{\mathrm{qo}} M$. Selon le corollaire 5.3.1, $M$ se décompose ainsi en $M=e_{\mathrm{qo}} M \oplus\left(1-e_{\mathrm{qo}}\right) M$ de telle sorte que les éléments de $\Lambda_{\mathrm{M}}^{+}$agissent par isomorphismes sur $e_{\mathrm{qo}} M$ alors qu'un élément, au moins, de $\Lambda_{\mathrm{M}}^{+}$agit par un endomorphisme topologiquement nilpotent $\operatorname{sur}\left(1-e_{\mathrm{qo}}\right) M$. Si h est un anneau et si $M$ est muni d'une structure de $\left(\overline{\mathcal{O}\left[\Lambda_{M}^{+}\right]}\right.$, h)-bimodule alors la décomposition précédente est une décomposition de h-module.

Puisque $\mathcal{O}$ est noethérien, les $\overline{\mathcal{O}\left[\Lambda_{\mathrm{M}}^{+}\right]}$-modules discrets sont exactement les limites inductives de $\mathcal{O}\left[\Lambda_{\mathrm{M}}^{+}\right]$-modules de type fini sur $\mathcal{O}$.

Par exemple, si $U \in \mathcal{T}_{\text {bp }}$ et si $L$ est un $\mathcal{O}\left[\left(U D_{*}\right)^{-1}\right]$-module de type fini sur $\mathcal{O}$ alors les groupes de cohomologie

$$
\mathrm{H}^{\cdot}\left(\mathcal{M}_{U K_{*}}, L\right) \quad \text { et } \quad \mathrm{H} \cdot\left(\mathcal{M}_{K_{*}}, L\right)
$$

sont des $\overline{\mathcal{O}\left[\Lambda_{\mathrm{M}}^{+}\right]}$-modules discrets. L'idempotent quasi-ordinaire $e_{\mathrm{qo}}$ décompose donc ces $\mathcal{H}\left(K_{*}, D_{*}\right)$-modules discrets. 


\subsubsection{Un lemme de Hida}

Nous montrons maintenant le lemme d'indépendance du niveau de la cohomologie $\mathbf{P}$-quasiordinaire. Il résulte des relations entre opérateurs de Hecke données au lemme 3.3.1. La méthode est due à Hida dans les cas $\mathbf{G}=\mathbf{G L}(2)(c f$. [18, (8.8)]) et $\mathbf{G}=\mathbf{G L}(n)(c f .[19,(4.7 b)])$.

LEMME 5.3.2. - Soit L un $\mathcal{O}\left[\left(U D_{0}\right)^{-1}\right]$-module de type fini sur $\mathcal{O}$.

Les applications canoniques $\mathrm{H}^{\cdot}\left(\mathcal{M}_{U K_{0}}, L\right) \rightarrow \mathrm{H}^{\cdot}\left(\mathcal{M}_{K_{0}}, L\right)$ induisent, sur les parties quasiordinaires, des isomorphismes de $\mathcal{H}_{\mathcal{O}}^{(p)}$-modules.

Démonstration. - D'après la proposition 5.2.1, il suffit de montrer les isomorphismes

$$
e_{\mathrm{qo}} \mathrm{H}^{\cdot}\left(U K_{0}, L\right) \stackrel{\sim}{\rightarrow} e_{\mathrm{qo}} \underline{\mathrm{H}}^{\cdot}\left(K_{0}, L\right) .
$$

Selon le lemme 3.3.1, pour tout sous-groupe $U^{\prime} \in \mathcal{T}_{\text {bp }}$ de $U$, il existe $\xi \in \sigma\left(\Lambda_{\mathbf{M}}^{+}\right)$tel que les triangles du diagramme

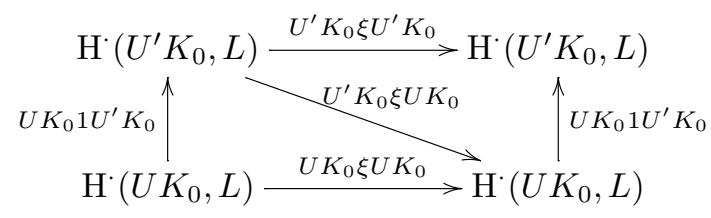

sont commutatifs. Puisque les opérateurs $U K_{0} \xi U K_{0}$ et $U^{\prime} K_{0} \xi U^{\prime} K_{0}$ sont des isomorphismes sur les parties quasi-ordinaires, les morphismes de restriction $U K_{0} 1 U^{\prime} K_{0}$ induisent, sur les parties quasi-ordinaires, des isomorphismes. On conclut par passage à la limite inductive sur les sousgroupes $U^{\prime} \in \mathcal{T}_{\text {bp }}$ de $U$.

\subsubsection{Suite spectrale quasi-ordinaire}

En corollaire du théorème 5.2.1 et du lemme précédent, on a la suite spectrale quasi-ordinaire :

Corollaire 5.3.2. - Soient $U \in \mathcal{T}_{\mathrm{bp}}$ et $L$ un $\mathcal{O}\left[\left(U D_{0}\right)^{-1}\right]$-module de type fini sur $\mathcal{O}$.

On a une suite spectrale $\mathcal{H}_{\mathcal{O}}^{(p)}$-équivariante :

$$
E_{2}^{i, j}=\mathrm{H}^{i}\left(C, e_{\mathrm{qo}} \mathrm{H}^{j}\left(\mathcal{M}_{K_{1}}, L\right)\right) \quad \Rightarrow \quad e_{\mathrm{qo}} \mathrm{H}^{i+j}\left(\mathcal{M}_{U K_{0}}, L\right) .
$$

Démonstration. - Puisque la projection quasi-ordinaire est un foncteur exact, il suffit de montrer que la cohomologie du groupe profini $C$ commute à la projection quasi-ordinaire :

$$
e_{\mathrm{qo}} \mathrm{H}^{\cdot}(C, L) \simeq \mathrm{H}^{\cdot}\left(C, e_{\mathrm{qo}} L\right)
$$

pour tout $\left(\overline{\mathcal{O}\left[\Lambda_{\mathbf{M}}^{+}\right]}, C\right)$-bimodule $L$ (discret pour $C$ ). Ce qui est immédiat grâce à la décomposition

$$
\mathrm{H}^{\cdot}(C, L)=\mathrm{H}^{\cdot}\left(C, e_{\mathrm{qo}} L\right) \oplus \mathrm{H}^{\cdot}\left(C,\left(1-e_{\mathrm{qo}}\right) L\right)
$$

puisque, $e_{\mathrm{qo}}$ agissant par l'identité sur $e_{\mathrm{qo}} L$ et, par l'application nulle sur $\left(1-e_{\mathrm{qo}}\right) L$, il en est de même sur leurs groupes de cohomologie respectifs.

\subsection{Théorème de contrôle abstrait}

Dans cette sous-section, nous démontrons le théorème de contrôle "abstrait". Pour cela, il faut d'abord établir quelques lemmes. 


\subsubsection{Version duale du lemme de Nakayama}

Le groupe abélien profini $C$ est produit d'un pro- $p$-groupe $C_{p}$ par un groupe fini $C^{(p)}$ d'ordre premier à $p$.

On note $\mathfrak{p}_{C}$ l'idéal premier noyau du morphisme d'augmentation

$$
\mathbb{Z}_{p}\left[\left[C_{p}\right]\right] \rightarrow \mathbb{Z}_{p}
$$

et $\mathfrak{m}_{C}$ l'idéal maximal $\mathfrak{m}_{C}:=\mathfrak{p}_{C}+p \mathbb{Z}_{p}\left[\left[C_{p}\right]\right]$.

LeMme 5.4.1. - La topologie de $\mathbb{Z}_{p}\left[\left[C_{p}\right]\right]$ coïncide avec la topologie $\mathfrak{m}_{C}$-adique.

Démonstration. - Par définition,

$$
\mathbb{Z}_{p}\left[\left[C_{p}\right]\right]=\varliminf_{n, U}^{\lim _{n, U}}\left(\mathbb{Z} / p^{n} \mathbb{Z}\right)\left[C_{p} / U\right]
$$

où $n$ parcourt $\mathbb{N}^{*}$ et $U$ parcourt l'ensemble des sous-groupes ouverts de $C_{p}$; il faut montrer que, pour de tels $n$ et $U$, le noyau de la projection naturelle $\left(\mathbb{Z} / p^{n} \mathbb{Z}\right)\left[C_{p} / U\right] \rightarrow \mathbb{Z} / p \mathbb{Z}$ est un idéal nilpotent.

Le noyau du morphisme $\left(\mathbb{Z} / p^{n} \mathbb{Z}\right)\left[C_{p} / U\right] \rightarrow(\mathbb{Z} / p \mathbb{Z})\left[C_{p} / U\right]$ étant nilpotent, on est ramené au cas $n=1$.

Et, si $p^{N}$ est l'ordre du $p$-groupe $C_{p} / U$ alors, dans $(\mathbb{Z} / p \mathbb{Z})\left[C_{p} / U\right]$, on a

$$
\forall \bar{\gamma} \in C_{p} / U, \quad(\bar{\gamma}-1)^{p^{N}}=\bar{\gamma}^{p^{N}}-1=0 .
$$

Ce qui montre bien que l'idéal d'augmentation de $(\mathbb{Z} / p \mathbb{Z})\left[C_{p} / U\right]$ est nilpotent.

Si $M$ est un module sur un anneau $A$ et $I$ un idéal de $A$, on note $M[I]$ le sous-module des éléments de $M$ annulés par $I$.

Lemme 5.4.2. - Soient A un anneau commutatif et I un idéal de A.

Munissant $A$ de la topologie I-adique, si $M$ est un A-module topologique discret et si $M[I]=0$, alors $M=0$.

Démonstration. - En appliquant $\operatorname{Hom}_{A}(\cdot, M)$ à la suite exacte courte

$$
0 \rightarrow I^{n} \otimes_{A} A / I \rightarrow A / I^{n+1} \rightarrow A / I^{n} \rightarrow 0
$$

où $n \in \mathbb{N}$, nous obtenons la suite exacte

$$
\operatorname{Hom}_{A}\left(I^{n}, M[I]\right) \leftarrow M\left[I^{n+1}\right] \leftarrow M\left[I^{n}\right] \leftarrow 0 .
$$

Ce qui montre $M\left[I^{n+1}\right]=M\left[I^{n}\right]$.

Or selon les hypothèses,

$$
M=\underset{n}{\lim } M\left[I^{n}\right] \quad \text { et } \quad M[I]=0,
$$

donc $M=0$.

\subsubsection{Cohomologie des $C$-modules discrets $p$-primaires}

LEMME 5.4.3. - Soient $p$ un nombre premier et $M$ un $C$-module discret p-primaire.

Si $\mathrm{H}^{0}(C, M)=0$ alors $\mathrm{H}^{0}\left(C^{(p)}, M\right)=0$ et $\mathrm{H}^{i}(C, M)=0$ pour tout $i$. 
Démonstration. - On pose $N=\mathrm{H}^{0}\left(C^{(p)}, M\right)$.

Si $\mathrm{H}^{0}(C, M)=0$ alors, a fortiori, $N\left[\mathfrak{m}_{C}\right]=0$ et le lemme 5.4.2 implique $N=0$.

Puisque $M$ est $p$-primaire, la suite spectrale de Hochschild-Serre donne immédiatement $\mathrm{H} \cdot(C, M)=\mathrm{H}^{\cdot}\left(C_{p}, N\right)=0$.

LEMME 5.4.4. - Soient p un nombre premier et $M$ un $C_{p}$-module discret p-primaire.

Si $M$ est de cotype fini sur $\mathbb{Z}_{p}\left[\left[C_{p}\right]\right]$ alors, pour tout sous-groupe fermé $C^{\prime}$ de $C_{p}$, les groupes $\mathrm{H}^{\cdot}\left(C^{\prime}, M\right)$ sont de cotype fini sur $\mathbb{Z}_{p}\left[\left[C_{p} / C^{\prime}\right]\right]$.

Démonstration. - Puisque $\mathbb{Z}_{p}\left[\left[C_{p}\right]\right]$ est noethérien, les sous-quotients des modules de cotype fini sont aussi de cotype fini. Ainsi, par dévissage du pro- $p$-groupe $C^{\prime}$, et grâce à la suite spectrale de Hochschild-Serre, on se ramène aux cas $C^{\prime}=\mathbb{Z}_{p}$ ou $C^{\prime}$ est un $p$-groupe.

Lorsque $C^{\prime}$ est fini, le résultat est évident.

Dans le premier cas, $C^{\prime}$ est de dimension cohomologique 1 et les groupes $\mathrm{H}^{0}\left(C^{\prime}, M\right)$ et $\mathrm{H}^{1}\left(C^{\prime}, M\right)$ sont respectivement ( $c f$. [31, chap. XIII, §1]) le noyau et le conoyau de l'homothétie de rapport $\gamma-1$ de $M$, où $\gamma$ est un générateur topologique de $C^{\prime}$. D'où les finitudes annoncées.

LEMME 5.4.5. - Soient p un nombre premier et $M$ un $C$-module discret p-primaire.

Si $\mathrm{H}^{0}(C, M)$ est fini, alors les groupes de cohomologie $\mathrm{H}^{i}(C, M)$ sont finis, pour tout $i$.

Démonstration. - On pose encore $N=\mathrm{H}^{0}\left(C^{(p)}, M\right)$.

$\mathrm{Si} \mathrm{H}^{0}(C, M)$ est fini alors, a fortiori, $N\left[\mathfrak{m}_{C}\right]$ et son dual $N^{\vee} /\left(\mathfrak{m}_{C} N^{\vee}\right)$ sont finis. Le lemme de Nakayama topologique montre que $N$ est un $\mathbb{Z}_{p}\left[\left[C_{p}\right]\right]$-module de cotype fini. Et selon le lemme 5.4.4, les groupes de cohomologie $\mathrm{H}^{\cdot}\left(C_{p}, N\right)=\mathrm{H}^{\cdot}(C, M)$ sont de cotype fini sur $\mathbb{Z}_{p}$. Pour avoir la finitude de ces groupes de cohomologie, il suffit donc de montrer qu'il sont annulés par une puissance de $p$.

La finitude de $\mathrm{H}^{0}(C, M)^{\vee}=N^{\vee} /\left(\mathfrak{p}_{C} N^{\vee}\right)$ donne aussi

$$
\left(N^{\vee} \otimes_{\mathbb{Z}_{p}\left[\left[C_{p}\right]\right]} \mathbb{Z}_{p}\left[\left[C_{p}\right]\right]_{\mathfrak{p}_{C}}\right) \otimes_{\mathbb{Z}_{p}\left[\left[C_{p}\right]\right]_{\mathfrak{p}_{C}}} \mathbb{Q}_{p}=N^{\vee} /\left(\mathfrak{p}_{C} N^{\vee}\right) \otimes_{\mathbb{Z}_{p}} \mathbb{Q}_{p}=0
$$

d'où, d'après le lemme de Nakayama, la nullité de $N^{\vee} \otimes_{\mathbb{Z}_{p}\left[\left[C_{p}\right]\right]} \mathbb{Z}_{p}\left[\left[C_{p}\right]\right]_{\mathfrak{m}_{C}}$. Ce qui signifie qu'il existe $f \in \mathbb{Z}_{p}\left[\left[C_{p}\right]\right]$ n'appartenant pas à $\mathfrak{p}_{C}$ qui annule $N^{\vee}$ (et $\left.N\right)$. La réduction de $f$ modulo $\mathfrak{p}_{C}$ annule donc la cohomologie $\mathrm{H}^{\cdot}\left(C_{p}, N\right)=\mathrm{H}^{\cdot}(C, M)$.

\subsubsection{Théorème de contrôle}

Dorénavant, $\mathcal{O}$ est intègre et de caractéristique résiduelle $p$. Soit $\mathcal{K}$ son corps des fractions. Si $L$ et $M$ sont deux $\mathcal{O}$-modules, on note parfois $L(M):=L \otimes_{\mathcal{O}} M$.

THÉORÈME 5.4.1. - Soient $U \in \mathcal{T}_{\mathrm{bp}}$ et $L$ un $\mathcal{O}\left[\left(U D_{0}\right)^{-1}\right]$-module, libre de type fini sur $\mathcal{O}$. Soit $q \in \mathbb{N}$ tel que

$$
\forall i<q, \quad e_{\mathrm{qo}} \mathrm{H}^{i}\left(\mathcal{M}_{U K_{0}}, L(\mathcal{K})\right)=0 \quad \text { et } \quad e_{\mathrm{qo}} \mathrm{H}^{i+1}\left(\mathcal{M}_{U K_{0}}, L\right)_{\text {tor }}=0 .
$$

Alors,

$$
\begin{gathered}
\forall i<q, \quad \mathrm{H}^{0}\left(C^{(p)}, e_{\mathrm{qo}} \mathrm{H}^{i}\left(\mathcal{M}_{K_{1}}, L(\mathcal{K} / \mathcal{O})\right)\right)=0, \\
e_{\mathrm{qo}} \mathrm{H}^{q}\left(\mathcal{M}_{U K_{0}}, L(\mathcal{K} / \mathcal{O})\right) \stackrel{\sim}{\rightarrow} \mathrm{H}^{0}\left(C, e_{\mathrm{qo}} \mathrm{H}^{q}\left(\mathcal{M}_{K_{1}}, L(\mathcal{K} / \mathcal{O})\right)\right)
\end{gathered}
$$

est un isomorphisme de $\mathcal{H}_{\mathcal{O}}^{(p)}$. 
Démonstration. - Selon le lemme 4.3.3, les hypothèses sont équivalentes à la nullité des $e_{\mathrm{qo}} \mathrm{H}^{i}\left(\mathcal{M}_{U K_{0}}, L(\mathcal{K} / \mathcal{O})\right)$, pour $i<q$.

La suite spectrale

$$
\mathrm{H}^{i}\left(C, e_{\mathrm{qo}} \mathrm{H}^{j}\left(\mathcal{M}_{K_{1}}, L(\mathcal{K} / \mathcal{O})\right)\right) \Rightarrow e_{\mathrm{qo}} \mathrm{H}^{i+j}\left(\mathcal{M}_{U K_{0}}, L(\mathcal{K} / \mathcal{O})\right)
$$

du corollaire 5.3.2 et le lemme 5.4.3 appliqués à $e_{\mathrm{qo}} \mathrm{H}^{j}\left(\mathcal{M}_{K_{1}}, L(\mathcal{K} / \mathcal{O})\right)$ donnent, par récurrence sur $j<q$, les annulations

$$
\begin{gathered}
\mathrm{H}^{0}\left(C^{(p)}, e_{\mathrm{qo}} \mathrm{H}^{j}\left(\mathcal{M}_{K_{1}}, L(\mathcal{K} / \mathcal{O})\right)\right)=0, \\
\mathrm{H}^{0}\left(C, e_{\mathrm{qo}} \mathrm{H}^{j}\left(\mathcal{M}_{K_{1}}, L(\mathcal{K} / \mathcal{O})\right)\right)=0 .
\end{gathered}
$$

Et la suite spectrale donne finalement l'isomorphisme annoncé.

THÉORÈME 5.4.2. - Soient $U \in \mathcal{T}_{\mathrm{bp}}$ et $L$ un $\mathcal{O}\left[\left(U D_{0}\right)^{-1}\right]$-module, libre de type fini sur $\mathcal{O}$. Soit $q \in \mathbb{N}$ tel que

$$
\forall i<q, \quad e_{\mathrm{qo}} \mathrm{H}^{i}\left(\mathcal{M}_{U K_{0}}, L(\mathcal{K})\right)=0
$$

Alors,

$$
\begin{gathered}
\forall i<q, \quad \mathrm{H}^{0}\left(C^{(p)}, e_{\mathrm{qo}} \mathrm{H}^{i}\left(\mathcal{M}_{K_{1}}, L(\mathcal{K} / \mathcal{O})\right)\right) \text { est fini, et } \\
e_{\mathrm{qo}} \mathrm{H}^{q}\left(\mathcal{M}_{U K_{0}}, L(\mathcal{K} / \mathcal{O})\right) \rightarrow \mathrm{H}^{0}\left(C, e_{\mathrm{qo}} \mathrm{H}^{q}\left(\mathcal{M}_{K_{1}}, L(\mathcal{K} / \mathcal{O})\right)\right)
\end{gathered}
$$

est un morphisme de $\mathcal{H}_{\mathcal{O}}^{(p)}$-modules, à noyau et conoyau finis.

Démonstration. - Selon le lemme 4.3.3 encore, les hypothèses sont équivalentes à la finitude $\operatorname{des} e_{\mathrm{qo}} \mathrm{H}^{i}\left(\mathcal{M}_{U K_{0}}, L(\mathcal{K} / \mathcal{O})\right)$, pour $i<q$.

La suite spectrale du corollaire 5.3.2 et le lemme 5.4.5 appliqués à

$$
e_{\mathrm{qo}} \mathrm{H}^{j}\left(\mathcal{M}_{K_{1}}, L(\mathcal{K} / \mathcal{O})\right)
$$

montrent, par récurrence sur $j<q$, la finitude de

$$
\mathrm{H}^{0}\left(C, e_{\mathrm{qo}} \mathrm{H}^{j}\left(\mathcal{M}_{K_{1}}, L(\mathcal{K} / \mathcal{O})\right)\right) .
$$

La suite spectrale donne donc le morphisme à noyau et conoyau finis annoncé.

\section{Algèbre de Hecke $p$-adique universelle}

Nous conservons les données de la section précédente et supposerons à partir du paragraphe 6.3.1 que le groupe adjoint $\mathbf{G}^{\text {ad }}$ possède des séries discrètes.

Cette dernière section est consacrée à l'algèbre de Hecke $\mathbf{P}$-quasi-ordinaire universelle $\mathbf{h}_{\mathrm{qo}}$. Cette algèbre dépend d'une représentation algébrique irréductible du groupe $\mathbf{G}$. C'est l'algèbre engendrée par les opérateurs diamants et les opérateurs de Hecke hors de $p$ agissant sur la cohomologie intérieure $\mathbf{P}$-quasi-ordinaire, de degré médian et de niveau infini de type $\Gamma_{1}\left(p^{\infty}\right)$, à valeurs dans un module $p$-adique construit à partir de la représentation fixée.

Un résultat d'annulation de la cohomologie en bas degré dû, indépendamment, à L. Saper [28], J.-S. Li et J. Schwermer [23], permet de déduire du théorème 5.4.2 de contrôle faible la finitude 
de $\mathbf{h}_{\mathrm{qo}}$ sur l'algèbre de Hida-Iwasawa $\Lambda$ (prop. 6.4.1). Sous une condition d'absence de $p$-torsion dans la cohomologie, nous déduisons aussi du théorème 5.4.1 de contrôle fort que la $\Lambda$-algèbre $\mathbf{h}_{\mathrm{qo}}$ est sans torsion (cor. 6.4.1). L'indépendance du poids établie au cor. 6.2.1 est un ingrédient commun à ces deux résultats.

En application, le dernier paragraphe est consacré à la construction de familles de systèmes de valeurs propres $\mathbf{P}$-quasi-ordinaires.

Ces résultats ont été montrés par Hida pour $\mathbf{G}=\mathbf{G L}(n)$ dans [20] et par Tilouine et Urban pour $\mathbf{G}=\mathbf{G S p}(4)$ dans [34].

Nous reprenons donc les notations et hypothèses de la section précédente, en supposant de plus que la partie bien placée $\Omega$ est réduite à un point spécial de l'immeuble, si bien que le sousgroupe spécial $\mathbf{G}_{\Omega}\left(\mathbb{Z}_{p}\right)$ donne lieu à une décomposition de Cartan. Nous fixons aussi l'anneau d'entiers $\mathcal{O}$ d'une sous-extension finie $\mathcal{K}$ d'une clôture algébrique $\overline{\mathbb{Q}_{p}}$ de $\mathbb{Q}_{p}$

- contenant les racines $n$-ièmes de l'unité, $n$ étant l'exposant (i.e. le plus petit commun multiple des ordres des éléments) de $C^{(p)}$;

- et qui déploie $\mathbf{G}_{\mathbb{Q}_{p}}$.

La première hypothèse servira pour établir la finitude de l'algèbre de Hecke quasi-ordinaire universelle sur l'algèbre de Hida-Iwasawa.

\subsection{Coefficients}

\subsubsection{Induction algébrique}

Nous renvoyons au livre de Jantzen [21] pour les résultats concernant les représentations algébriques.

Soit $\rho: \mathbf{M}_{\Omega} \rightarrow \mathbf{G L}(V(\mathcal{O}))$ une représentation $\mathcal{O}$-algébrique de $\mathbf{M}_{\Omega}$ sur un $\mathcal{O}$-module libre de rang fini $V(\mathcal{O})$. On l'identifie à la représentation de $\mathbf{P}_{\Omega}$, triviale sur $\mathbf{U}_{\Omega}$, obtenue par composition avec la projection naturelle $\mathbf{P}_{\Omega} \rightarrow \mathbf{M}_{\Omega}$.

Soit $L(\rho, \mathcal{K}):=\operatorname{ind}_{\mathbf{P}_{\mathcal{K}}}^{\mathbf{G}_{\mathcal{K}}} \rho$ le module de la représentation $\mathcal{K}$-algébrique de $\mathbf{G}$ induite [21, I.3.3] à partir de $\rho$. En notant $\mathcal{K}(\mathbf{G})$ la $\mathcal{K}$-algèbre des fonctions $\mathcal{K}$-algébriques sur $\mathbf{G}_{\mathcal{K}}, L(\rho, \mathcal{K})$ est le $\mathcal{K}$-espace vectoriel formé des fonctions $f \in \mathcal{K}(\mathbf{G}) \otimes_{\mathcal{O}} V(\mathcal{O})$ telles que

$$
\forall(g, t, n) \in \mathbf{G}(\mathcal{K}) \times \mathbf{M}(\mathcal{K}) \times \mathbf{U}(\mathcal{K}), \quad f(g t n)=\rho(t)^{-1} f(g)
$$

muni de l'action algébrique donnée par $(g f)(h)=f\left(g^{-1} h\right)$ quels que soient $f \in L(\rho, \mathcal{K})$ et $g, h \in \mathbf{G}(\mathcal{K})$.

On suppose que $\rho$, en tant que représentation $\mathcal{K}$-algébrique de $\mathrm{M}$, est irréductible et que le module induit $L(\rho, \mathcal{K})$ est non nul.

D'après [14, II.2.3.1], une représentation algébrique irréductible sur un corps est toujours de dimension finie. De plus, les représentations algébriques d'un groupe réductif sur un corps de caractéristique nulle sont semi-simples ( $c f$. [21, II.5.6]).

Puisque $\mathbf{G}_{\mathcal{K}}$ est déployé, il existe un tore maximal $\mathbf{T}$ de $\mathbf{Z}$ défini et déployé sur $\mathcal{K}$. C'est aussi un tore maximal de $\mathbf{G}$, contenu dans $\mathbf{M}$. On choisit un sous-groupe de Borel $\mathbf{B}^{-}$de $\mathbf{G}$ défini sur $\mathcal{K}$, contenu dans $\mathbf{P}^{-}$et contenant $\mathbf{T}$.

Selon [21, II.2.1-7], la représentation $\rho$ étant irréductible, elle possède un plus grand poids $\chi_{\rho} \in \mathrm{X}^{*}(\mathbf{T})$ dominant pour l'ordre défini par le sous-groupe de Borel $\mathbf{B}^{-} \cap \mathbf{M}$ de $\mathbf{M}$. L'induite $L(\rho, \mathcal{K})$ est non nulle si et seulement si ce caractère est même dominant pour l'ordre défini par $\mathbf{B}^{-}$. Dans ce cas, $L(\rho, \mathcal{K})$ est aussi irréductible et $\chi_{\rho}$ est aussi son plus grand poids. 


\subsubsection{Construction d'un réseau}

Soit $U \in \mathcal{T}_{\mathrm{bp}}$. Nous allons maintenant plonger $L(\rho, \mathcal{K})$ dans un ensemble de fonctions continues sur un espace $p$-adique $Y_{U}$ et définir un réseau. Considérons le sous-espace $p$-adique

$$
Y_{U}:=U \mathbf{M}_{\Omega}\left(\mathbb{Z}_{p}\right) \mathbf{U}\left(\mathbb{Q}_{p}\right) / \mathbf{U}\left(\mathbb{Q}_{p}\right) \subset \mathbf{G}\left(\mathbb{Q}_{p}\right) / \mathbf{U}\left(\mathbb{Q}_{p}\right) .
$$

L'espace $U$ étant un ouvert $p$-adique de $\mathbf{G}\left(\mathbb{Q}_{p}\right)$, il est Zariski-dense dans $\mathbf{G}(\mathcal{K})$. D'où l'injection de la première ligne dans le carré cartésien

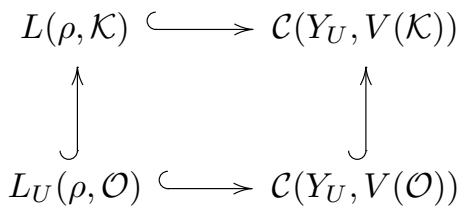

définissant le réseau $L_{U}(\rho, \mathcal{O}) \subset L(\rho, \mathcal{K})$. Le $\mathcal{O}$-module conoyau de la seconde ligne est sans torsion (donc plat) car il s'injecte dans le $\mathcal{K}$-espace vectoriel conoyau de la première ligne.

Pour tout $\mathcal{O}$-module $M$, on note $V(M):=V(\mathcal{O}) \otimes_{\mathcal{O}} M$ et

$$
L_{U}(\rho, M):=L_{U}(\rho, \mathcal{O}) \otimes_{\mathcal{O}} M \hookrightarrow \mathcal{C}\left(Y_{U}, V(\mathcal{O})\right) \otimes_{\mathcal{O}} M .
$$

Ainsi on a

$$
\begin{gathered}
L_{U}(\rho, \mathcal{K})=L(\rho, \mathcal{K}), \\
L_{U}(\rho, \mathcal{K} / \mathcal{O})=L(\rho, \mathcal{K}) / L_{U}(\rho, \mathcal{O})=\lim _{r} L_{U}\left(\rho, p^{-r} \mathcal{O} / \mathcal{O}\right) .
\end{gathered}
$$

\subsubsection{Action par conjugaison}

Pour $U \in \mathcal{T}_{\mathrm{bp}}$, nous décrivons maintenant une première action du monoïde $\mathbf{R M}\left(\mathbb{Q}_{p}\right)^{+}$(défini en 2.4) sur $Y_{U}$. L'action $(\xi, g) \mapsto \xi g$ de $\mathbf{M}\left(\mathbb{Q}_{p}\right)$ par conjugaison sur $\mathbf{G}\left(\mathbb{Q}_{p}\right)$ passe au quotient $\mathbf{G}\left(\mathbb{Q}_{p}\right) / \mathbf{U}\left(\mathbb{Q}_{p}\right)$. La décomposition (13) et la propriété (14) montrent que le sous-ensemble

$$
Y_{U}=\left(U \cap \mathbf{U}_{\Omega}^{-}\left(\mathbb{Z}_{p}\right)\right) \mathbf{M}_{\Omega}\left(\mathbb{Z}_{p}\right) \mathbf{U}\left(\mathbb{Q}_{p}\right) / \mathbf{U}\left(\mathbb{Q}_{p}\right) \subset \mathbf{G}\left(\mathbb{Q}_{p}\right) / \mathbf{U}\left(\mathbb{Q}_{p}\right)
$$

est stable par le sous-monoïde $\mathbf{R M}\left(\mathbb{Q}_{p}\right)^{+}$de $\mathbf{M}\left(\mathbb{Q}_{p}\right)$.

Remarquons que, pour $\xi \in \mathbf{R M}\left(\mathbb{Q}_{p}\right) \cap \mathbf{M}_{\Omega}\left(\mathbb{Z}_{p}\right)$, l'action ainsi définie ne coïncide pas avec l'action de $U \mathbf{P}_{\Omega}\left(\mathbb{Z}_{p}\right)$ par multiplication à gauche sur $Y_{U}$. Afin d'étendre l'action de $U \mathbf{P}_{\Omega}\left(\mathbb{Z}_{p}\right)$ sur $Y_{U}$ en une action du monoïde $U D_{0, p}$ nous devons faire un choix et tordre cette action.

\subsubsection{Modification de l'action}

On définit le morphisme de monoïdes

$$
\begin{aligned}
\delta: U D_{0, p} \rightarrow & U \mathbf{P}_{\Omega}\left(\mathbb{Z}_{p}\right) \backslash U D_{0, p} / U \mathbf{P}_{\Omega}\left(\mathbb{Z}_{p}\right) \stackrel{\sim}{\leftarrow} \sigma\left(\Lambda_{\mathbf{M}}^{+}\right) \\
u \xi p \mapsto U \mathbf{P}_{\Omega}\left(\mathbb{Z}_{p}\right) \xi U \mathbf{P}_{\Omega}\left(\mathbb{Z}_{p}\right) & \mapsto \delta(u \xi p)=\xi
\end{aligned}
$$

$\operatorname{par} \delta(u \xi p)=\xi$ où $u \in U, \xi \in \sigma\left(\Lambda_{\mathbf{M}}^{+}\right)$et $p \in \mathbf{P}_{\Omega}\left(\mathbb{Z}_{p}\right)$. C'est une rétraction de l'inclusion $\sigma\left(\Lambda_{\mathbf{M}}^{+}\right) \subset U D_{0, p}$. On étend l'action par multiplication à gauche de $U \mathbf{P}_{\Omega}\left(\mathbb{Z}_{p}\right)$ sur $\mathbf{G}\left(\mathbb{Q}_{p}\right)$ en une action, notée $*$, de $U D_{0, p}$ par $\xi * g:=\xi g \delta(\xi)^{-1}$. Cette action passe au quotient $\mathbf{G}\left(\mathbb{Q}_{p}\right) / \mathbf{U}\left(\mathbb{Q}_{p}\right)$ et, d'après le paragraphe précédent, elle stabilise $Y_{U}$. 


\subsubsection{Action sur les espaces de fonctions}

Ainsi, pour tout $\mathcal{O}$-module $M$, l'espace de fonctions $\mathcal{C}\left(Y_{U}, M\right)$ est un module sur $\left(U D_{0, p}\right)^{-1}$ :

$$
\left(\xi^{-1} * f\right)\left(g \mathbf{U}\left(\mathbb{Q}_{p}\right)\right):=f\left(\xi * g \mathbf{U}\left(\mathbb{Q}_{p}\right)\right)=f\left(\xi g \delta(\xi)^{-1} \mathbf{U}\left(\mathbb{Q}_{p}\right)\right)
$$

quels que soient $f \in \mathcal{C}\left(Y_{U}, M\right), \xi \in U D_{0, p}$ et $g \mathbf{U}\left(\mathbb{Q}_{p}\right) \in Y_{U}$.

De plus $L_{U}(\rho, M) \subset \mathcal{C}\left(Y_{U}, M\right)$ est un sous- $\mathbb{Z}\left[\left(U D_{0, p}\right)^{-1}\right]$-module car $\sigma\left(\Lambda_{\mathbf{M}}^{+}\right)$est central dans $\mathbf{M}\left(\mathbb{Q}_{p}\right)$.

On remarque que si $f \in L(\rho, \mathcal{K})$, on a $\xi^{-1} * f=\left(\omega_{\rho} \circ \delta\right)(\xi)\left(\xi^{-1} f\right)$, où $\omega_{\rho} \in \mathrm{X}^{*}(\mathbf{R M})$ est le caractère central de $\rho$.

\subsubsection{Décomposition selon l'action de $R M$}

Puisque $\mathbf{G}$ est déployé sur $\mathcal{K}$, le radical $\mathbf{R M}$ de $\mathbf{M}$ est un tore $\mathcal{K}$-déployé, si bien que la représentation algébrique induite, $L(\rho, \mathcal{K})$, se décompose, en tant que représentation algébrique de $\mathbf{M}$, sous la forme d'une somme directe

$$
L(\rho, \mathcal{K})=\bigoplus_{\chi \in \mathrm{X}^{*}(\mathbf{R M})} L(\rho, \mathcal{K})\left[\omega_{\rho} \chi\right]
$$

où $\mathbf{R M}$ agit sur la composante $L(\rho, \mathcal{K})\left[\omega_{\rho} \chi\right]$ par le caractère $\omega_{\rho} \chi$.

Soit $\mathrm{X}^{*}(\mathbf{R M})^{+}$le sous-monoïde des caractères de $\mathbf{R M}$ qui sont combinaison linéaire des caractères intervenant dans la représentation adjointe de $\mathbf{R M}$ sur l'algèbre de Lie de $\mathbf{U}$.

Puisque le plus grand poids $\chi_{\rho}$, pour l'ordre défini par $\mathbf{B}^{-}$, de $L(\rho, \mathcal{K})$ coïncide sur $\mathbf{R M}$ avec le caractère central $\omega_{\rho}$ de $\rho$, seules les composantes correspondant aux caractères $\chi \in \mathrm{X}^{*}(\mathbf{R M})^{+}$ peuvent être non nulles.

Selon la formule (21), on a $t^{-1} * f=\chi(t)^{-1} f$ pour tout $t \in \sigma\left(\Lambda_{\mathbf{M}}^{+}\right)$et tout $f \in L(\rho, \mathcal{K})\left[\omega_{\rho} \chi\right]$. De plus, pour tout caractère $\chi \in \mathrm{X}^{*}(\mathbf{R M})^{+}$, par définition de $\mathbf{R M}\left(\mathbb{Q}_{p}\right)^{+}(c f$. égalité $(11)$ ), on a $\omega(\chi(t)) \leqslant 0, \omega$ étant la valuation de $\mathcal{K}$.

\subsection{Indépendance du poids}

On fait agir les monoïdes $U^{\prime} D_{0}\left(U^{\prime} \in \mathcal{T}_{\mathrm{bp}}\right)$ à travers la projection sur la composante p-adique $U^{\prime} D_{0} \rightarrow U^{\prime} D_{0, p}$

\subsubsection{Caractères $\mathcal{O}$-arithmétiques}

Un caractère $\mathcal{O}$-arithmétique de $\mathbf{M}_{\Omega}$ est un caractère $p$-adique continu

$$
\mathbf{M}_{\Omega}\left(\mathbb{Z}_{p}\right) \rightarrow \mathcal{O}^{\times}
$$

qui coïncide, sur un voisinage de l'unité, avec un caractère $\mathcal{O}$-algébrique de $\mathbf{M}_{\Omega}$. Un tel caractère s'écrit de manière unique comme le produit $\varepsilon \chi$ :

- d'un caractère $\mathcal{O}$-algébrique $\chi$ de $\mathbf{M}_{\Omega}$,

- et d'un caractère fini continu $\varepsilon: \mathbf{M}_{\Omega}\left(\mathbb{Z}_{p}\right) \rightarrow \mathcal{O}^{\times}$.

On note $\mathcal{O}(\varepsilon \chi)$ la $\mathcal{O}[[C]]$-algèbre dont la $\mathcal{O}$-algèbre sous-jacente est $\mathcal{O}$, munie du morphisme structural $\mathcal{O}[[C]] \rightarrow \mathcal{O}$ induit par le caractère $(\varepsilon \chi)^{-1}$. Soit $\mathfrak{p}_{\varepsilon \chi}$ l'idéal premier noyau de ce morphisme.

Pour tout $\mathcal{O}[[C]]$-module (resp. toute $\mathcal{O}[[C]]$-algèbre) $M$, on notera $M(\varepsilon \chi)$ le $\mathcal{O}[[C]]$-module (resp. $\mathcal{O}[[C]]$-algèbre) tordu par $\chi$ :

$$
M(\varepsilon \chi):=M \otimes_{\mathcal{O}} \mathcal{O}(\varepsilon \chi) .
$$


On notera aussi, pour un $\mathcal{O}[[C]]$-module $M$ :

$$
M[\varepsilon \chi]:=\mathrm{H}^{0}(C, M(\varepsilon \chi))=\{m \in M \mid \forall c \in C, c m=(\varepsilon \chi)(c) m\} .
$$

On dit que le caractère $\varepsilon \chi$ est dominant par rapport à $\rho$ si et seulement si le module $L(\rho \otimes \chi, \mathcal{K})$ est non nul, c'est-à-dire si le caractère $\chi \chi_{\rho}$ de $\mathbf{T}$ est dominant pour l'ordre défini $\operatorname{par} \mathbf{B}^{-}$.

\subsubsection{Torsion des coefficients par un caractère fini}

On note $\mathcal{T}_{\mathrm{bp}}(\varepsilon)$ l'ensemble des sous-groupes $U^{\prime} \in \mathcal{T}_{\mathrm{bp}}$ tels que le caractère continu fini $\varepsilon$ se factorise à travers $C_{U^{\prime}}$, i.e. $U^{\prime} \cap \mathbf{M}_{\Omega}\left(\mathbb{Z}_{p}\right) \subset \operatorname{ker} \varepsilon$.

Pour un tel $U^{\prime}$, $\varepsilon$ peut être étendu de manière unique en un caractère (encore noté $\varepsilon$ ) de $U^{\prime} D_{0, p}$ par la formule $\varepsilon(u t m n)=\varepsilon(m)$, quels que soient $u \in U^{\prime}, t \in \sigma\left(\Lambda_{\mathbf{M}}^{+}\right), m \in \mathbf{M}_{\Omega}\left(\mathbb{Z}_{p}\right)$ et $n \in \mathbf{U}_{\Omega}\left(\mathbb{Z}_{p}\right)$. Ce caractère est trivial sur $U^{\prime} D_{1, p}$.

Si $U \in \mathcal{T}_{\text {bp }}$ contient $U^{\prime}, L_{U}(\rho \otimes \varepsilon \chi, \cdot)$ désigne le $\mathbb{Z}\left[\left(U^{\prime} D_{0, p}\right)^{-1}\right]$-module obtenu à partir de $L_{U}(\rho \otimes \chi, \cdot)$ en tordant l'action de $\left(U^{\prime} D_{0, p}\right)$ par le caractère $\varepsilon$ de la manière définie au paragraphe 4.3.7.

\subsubsection{Torsion des coefficients par un caractère $\mathcal{O}$-algébrique}

Pour tout $r \geqslant 0$, soit $\mathcal{T}_{\mathrm{bp}}(r)$ l'ensemble des sous-groupes $U^{\prime} \in \mathcal{T}_{\mathrm{bp}}$ tels que $U^{\prime} \cap \mathbf{M}_{\Omega}\left(\mathbb{Z}_{p}\right)$ est contenu dans le sous-groupe de congruence principal noyau du morphisme de réduction $\mathbf{M}_{\Omega}\left(\mathbb{Z}_{p}\right) \rightarrow \mathbf{M}_{\Omega}\left(\mathbb{Z} / p^{r} \mathbb{Z}\right)$.

Pour un tel sous-groupe $U^{\prime}$, toute action de $\mathbf{M}_{\Omega}\left(\mathbb{Z} / p^{r} \mathbb{Z}\right)$ peut s'étendre en une action du monoïde $\left(U^{\prime} D_{0, p}\right)^{-1}$ au moyen de l'application

$$
\begin{aligned}
U^{\prime} D_{0, p} & \rightarrow \mathbf{M}_{\Omega}\left(\mathbb{Z} / p^{r} \mathbb{Z}\right) \\
\text { utmn } & \mapsto m \bmod p^{r}
\end{aligned}
$$

quels que soient $u \in U^{\prime}, t \in \sigma\left(\Lambda_{\mathbf{M}}^{+}\right), m \in \mathbf{M}_{\Omega}\left(\mathbb{Z}_{p}\right)$ et $n \in \mathbf{U}_{\Omega}\left(\mathbb{Z}_{p}\right)$.

On étend ainsi l'action $\rho$ de $\mathbf{M}_{\Omega}\left(\mathbb{Z} / p^{r} \mathbb{Z}\right)$ sur $V\left(p^{-r} \mathcal{O} / \mathcal{O}\right)$ en une action du monoïde $\left(U^{\prime} D_{0, p}\right)^{-1}$.

De plus, on note $V\left(p^{-r} \mathcal{O} / \mathcal{O}\right)(\chi)$ le même module muni de l'action tordue de $\left(U^{\prime} D_{0, p}\right)^{-1}$ définie $\operatorname{par}(u t m n)^{-1} \mapsto(\rho \otimes \chi)(m)^{-1} \bmod p^{r}$.

Proposition 6.2.1. - Soit $\chi$ un caractère $\mathcal{O}$-algébrique $\rho$-dominant de $\mathbf{M}_{\Omega}$.

Pour tout $U \in \mathcal{T}_{\mathrm{bp}}$, on a un isomorphisme naturel de $\mathcal{H}^{(p)}[[C]]$-modules

$$
e_{\mathrm{qo}} \mathrm{H}_{*}^{\cdot}\left(\mathcal{M}_{K_{1}}, L_{U}(\rho \otimes \chi, \mathcal{K} / \mathcal{O})\right) \simeq \underline{\lim }_{r, U^{\prime}} e_{\mathrm{qo}} \mathrm{H}_{*}^{\cdot}\left(\mathcal{M}_{U^{\prime} K_{1}}, V\left(p^{-r} \mathcal{O} / \mathcal{O}\right)\right)(\chi)
$$

où la limite inductive porte sur $r \geqslant 0$ et sur les sous-groupes $U^{\prime} \in \mathcal{T}_{\mathrm{bp}}(r)$ de $U$ et $\mathrm{H}_{*}$ désigne la cohomologie totale, du bord, à support compact ou bien intérieure de $\mathcal{M}$.

Démonstration. - L'application canonique de l'induite

$$
L(\rho, \mathcal{K}) \rightarrow V(\mathcal{K})
$$

induit le morphisme d'évaluation au point $1 \mathbf{U}\left(\mathbb{Q}_{p}\right) \in Y_{U}$ :

$$
L_{U}(\rho \otimes \chi, \mathcal{K} / \mathcal{O}) \subset \mathcal{C}\left(Y_{U}, V(\mathcal{K} / \mathcal{O})\right) \rightarrow V(\mathcal{K} / \mathcal{O})
$$


Pour tout sous-groupe $U^{\prime} \in \mathcal{T}_{\text {bp }}$ de $U$, l'action de $U D_{0, p}$ sur $Y_{U}$ passe au quotient $Y_{U / U^{\prime}}$ défini par

$$
Y_{U / U^{\prime}}:=U \mathbf{P}_{\Omega}\left(\mathbb{Z}_{p}\right) / U^{\prime} \mathbf{U}_{\Omega}\left(\mathbb{Z}_{p}\right)=U^{\prime} \backslash U \mathbf{M}_{\Omega}\left(\mathbb{Z}_{p}\right) \mathbf{U}\left(\mathbb{Q}_{p}\right) / \mathbf{U}\left(\mathbb{Q}_{p}\right) .
$$

Ainsi le sous-espace des fonctions qui passent au quotient :

$$
L_{U / U^{\prime}}(\rho \otimes \chi, \cdot):=L_{U}(\rho \otimes \chi, \cdot) \cap \mathcal{C}\left(Y_{U / U^{\prime}}, \cdot\right)
$$

est stable sous $\left(U D_{0}\right)^{-1}$. Puisque

$$
U \mathbf{P}_{\Omega}\left(\mathbb{Z}_{p}\right)=\varliminf_{U^{\prime} \in \mathcal{T}_{\mathrm{bp}}, U^{\prime} \subset U} U \mathbf{P}_{\Omega}\left(\mathbb{Z}_{p}\right) / U^{\prime}
$$

est profini et $\mathcal{K} / \mathcal{O}$ est discret, le morphisme d'évaluation précédent est la limite inductive (indexée par $r \geqslant 0$ et $\left.U^{\prime} \in \mathcal{T}_{\mathrm{bp}}(r), U^{\prime} \subset U\right)$ des morphismes d'évaluation en $1 U^{\prime} \mathbf{U}_{\Omega}\left(\mathbb{Z}_{p}\right) \in$ $Y_{U / U^{\prime}}$ suivants :

$$
L_{U / U^{\prime}}\left(\rho \otimes \chi, p^{-r} \mathcal{O} / \mathcal{O}\right) \rightarrow V\left(p^{-r} \mathcal{O} / \mathcal{O}\right)(\chi)
$$

On remarque que ces morphismes sont $\left(U^{\prime} D_{0}\right)^{-1}$-équivariants.

Pour $U^{\prime} \subset U$ fixés, selon le lemme 3.3.1 et la définition de $Y_{U / U^{\prime}}$ donnée plus haut, il existe $t \in \sigma\left(\Lambda_{\mathbf{M}}^{+}\right)$tel que $t * Y_{U / U^{\prime}}=U^{\prime} \mathbf{P}_{\Omega}\left(\mathbb{Z}_{p}\right) /\left(U^{\prime} \mathbf{U}_{\Omega}\left(\mathbb{Z}_{p}\right)\right)$. Le noyau du morphisme d'évaluation précédent est donc annulé par ce $t$. D'où l'isomorphisme

$$
e_{\mathrm{qo}} \mathrm{H}_{*}\left(\mathcal{M}_{U^{\prime} K_{1}}, L_{U / U^{\prime}}\left(\rho \otimes \chi, p^{-r} \mathcal{O} / \mathcal{O}\right)\right) \simeq e_{\mathrm{qo}} \mathrm{H}_{*}\left(\mathcal{M}_{U^{\prime} K_{1}}, V\left(p^{-r} \mathcal{O} / \mathcal{O}\right)(\chi)\right) .
$$

En passant à la limite inductive, et en utilisant le lemme 4.3.2, on obtient l'isomorphisme

$$
e_{\mathrm{qo}} \mathrm{H}_{*}^{\cdot}\left(\mathcal{M}_{K_{1}}, L_{U}(\rho \otimes \chi, \mathcal{K} / \mathcal{O})\right) \simeq \lim _{r, U^{\prime}} e_{\mathrm{qo}} \mathrm{H}_{*}^{\cdot}\left(\mathcal{M}_{U^{\prime} K_{1}}, V\left(p^{-r} \mathcal{O} / \mathcal{O}\right)\right)(\chi)
$$

où la limite inductive porte sur les $r \geqslant 0$ et les sous-groupes $U^{\prime} \in \mathcal{T}_{\mathrm{bp}}(r)$ de $U$. En effet, dans le membre de droite, on a pu intervertir le passage aux parties quasi-ordinaires et la torsion par $\chi$ car le caractère de $U^{\prime} D_{0, p}$, étendu à partir de $\chi$, par lequel on tord, est trivial sur $\sigma\left(\Lambda_{\mathbf{M}}^{+}\right)$.

On note $\mathrm{h}_{*, U, \text { qo }}\left(K_{1}, \rho \otimes \varepsilon \chi\right)$ la sous- $\mathcal{O}[[C]]$-algèbre engendrée par l'image de l'anneau de Hecke abstrait $\mathcal{H}^{(p)}$ dans l'algèbre des endomorphismes du $\mathcal{O}[[C]]$-module

$$
e_{\mathrm{qo}} \mathrm{H}_{*}\left(\mathcal{M}_{K_{1}}, L_{U}(\rho \otimes \varepsilon \chi, \mathcal{K} / \mathcal{O})\right) .
$$

Corollaire 6.2.1. - Soit $\varepsilon \chi$ un caractère $\mathcal{O}$-arithmétique $\rho$-dominant de $\mathbf{M}_{\Omega}$.

Pour tout $U \in \mathcal{T}_{\mathrm{bp}}(\varepsilon)$, on a un isomorphisme de $\mathcal{H}^{(p)}[[C]]$-modules

$$
e_{\mathrm{qo}} \mathrm{H}_{*}\left(\mathcal{M}_{K_{1}}, L_{U}(\rho \otimes \varepsilon \chi, \mathcal{K} / \mathcal{O})\right) \simeq e_{\mathrm{qo}} \mathrm{H}_{*}\left(\mathcal{M}_{K_{1}}, L_{U}(\rho, \mathcal{K} / \mathcal{O})\right)(\varepsilon \chi) .
$$

Ce qui induit un isomorphisme de $\mathcal{O}[[C]]$-algèbres :

$$
\mathrm{h}_{*, U, \mathrm{qo}}\left(K_{1}, \rho \otimes \varepsilon \chi\right) \simeq \mathrm{h}_{*, U, \mathrm{qo}}^{\cdot}\left(K_{1}, \rho\right)(\varepsilon \chi) .
$$

Démonstration. - Par définition du module $L_{U}(\rho \otimes \varepsilon \chi)$ et d'après le lemme 4.3.2, on a

$$
e_{\mathrm{qo}} \mathrm{H}_{*}\left(\mathcal{M}_{K_{1}}, L_{U}(\rho \otimes \varepsilon \chi, \mathcal{K} / \mathcal{O})\right) \simeq e_{\mathrm{qo}} \mathrm{H}_{*}\left(\mathcal{M}_{K_{1}}, L_{U}(\rho \otimes \chi, \mathcal{K} / \mathcal{O})\right)(\varepsilon) .
$$

Ce corollaire est donc une conséquence immédiate de la proposition précédente. 


\subsection{Résultats d'annulation de la cohomologie}

On suppose dorénavant que $\mathcal{K}$ est le complété, en une place au-dessus de $p$, d'un corps de nombre $\mathcal{K}_{0}$ qui déploie $\mathbf{G}$.

\subsubsection{Hypothèse de Harish-Chandra}

Nous supposons aussi désormais que le groupe adjoint $\mathbf{G}^{\text {ad }}$ de $\mathbf{G}$ possède des séries discrètes. Selon Harish-Chandra, cette hypothèse équivaut à l'égalité entre le rang (absolu) de $\mathbf{G}^{\text {ad }}$ et celui de ses sous-groupes compacts maximaux $K_{\mathbb{R}}^{\text {ad }} \subset \mathrm{G}^{\text {ad }}(\mathbb{R})$.

Puisque la dimension et le rang réductif (absolu) d'un groupe réductif ont même parité (comme on le voit sur la grosse cellule), l'existence de séries discrètes pour $\mathbf{G}^{\text {ad }}$ implique que son domaine symétrique $X^{\text {ad }}$ est de dimension (réelle) paire. Nous noterons $2 d$ cette dimension.

De plus, l'existence de séries discrètes est vérifiée, par exemple, lorsque $\mathbf{G}$ admet une donnée de Shimura $h: \mathbf{R}_{\mathbb{C} / \mathbb{R}} \mathbf{G m} \rightarrow \mathbf{G}_{\mathbb{R}}$. En effet, tout tore maximal de $\mathbf{G}_{\mathbb{R}}^{\text {ad }}$ contenant l'image de $h$ est inclus dans le centralisateur de $h(i)$ dans $\mathbf{G}^{\text {ad }}(\mathbb{R})$, qui est, selon un des axiomes de Deligne [13], un sous-groupe compact maximal.

\subsubsection{Finitude}

Pour pouvoir appliquer le théorème de contrôle "faible" 5.4.2, on utilise ici un résultat d'annulation de la cohomologie, dû indépendamment à L. Saper [28, th. 5] d'une part, J.-S. Li et J. Schwermer [23] d'autre part.

THÉORÈME 6.3.1 [23,28]. - Soit $\mathbf{G}$ un groupe réductif connexe défini sur $\mathbb{Q}$, dont le sous-tore $\mathbb{R}$-déployé central maximal est $\mathbb{Q}$-déployé (i.e. $r_{\mathbf{R G}}=0$ ) et dont le groupe adjoint possède des séries discrètes.

Soit $V(\mathbb{C})$ une représentation complexe irréductible, de dimension finie et de plus haut poids régulier.

Pour tout sous-groupe ouvert compact $U \subset \mathbf{G}\left(\mathbb{A}_{\mathrm{f}}\right)$, on a

$$
\forall i<d, \quad \mathrm{H}^{i}\left(\mathcal{M}_{U}, V(\mathbb{C})\right)=0
$$

où d est la moitié de la dimension (réelle) du domaine symétrique de $\mathbf{G}$.

Saper dérive ce résultat de sa théorie des $\mathcal{L}$-modules [27]. Tandis que Li et Schwermer le déduisent de leur étude de la contribution des séries d'Eisenstein à la cohomologie des groupes arithmétiques.

On note $\mathbf{R}_{\mathbb{R}}^{\mathbb{Q}} \mathbf{G}$ le plus grand sous-tore $\mathbb{Q}$-anisotrope de $\mathbf{R}_{\mathbb{R}} \mathbf{G}$. Il est de dimension $r_{\mathbf{R G}}$ et contient $\Gamma_{K} \cap \mathbf{R}_{\mathbb{R}} G(\mathbb{R})^{+}$lorsque $K \in \mathcal{T}$.

Grâce au corollaire 4.3.1, on en déduit le

Corollaire 6.3.1. - Soit $\mathbf{G}$ un groupe réductif connexe défini sur $\mathbb{Q}$ dont le groupe adjoint possède des séries discrètes.

Soit $V(\mathbb{C})$ une représentation complexe irréductible, de dimension finie, de plus haut poids régulier et de caractère central trivial sur $\mathbf{R}_{\mathbb{R}}^{\mathbb{Q}} \mathbf{G}$.

Pour tout sous-groupe ouvert compact $U \subset \mathbf{G}\left(\mathbb{A}_{\mathrm{f}}\right)$, on a

$$
\begin{gathered}
\forall i<d, \quad \mathrm{H}^{i}\left(\mathcal{M}_{U}, V(\mathbb{C})\right)=0 \\
\text { et } \quad \mathrm{H}^{d}\left(\mathcal{M}_{U}, V(\mathbb{C})\right)=\mathrm{H}^{d}\left(\mathcal{M}_{U}^{\mathrm{ad}}, V(\mathbb{C})\right)
\end{gathered}
$$

où d est la moitié de la dimension (réelle) du domaine symétrique de $\mathbf{G}$. 
THÉORÈmE 6.3.2. - Soient $\rho$ une représentation $\mathcal{K}$-algébrique irréductible de $\mathbf{M}$ de plus haut poids régulier, trivial sur $\mathbf{R}_{\mathbb{R}}^{\mathbb{Q}} \mathbf{G}$. Soit $U \in \mathcal{T}_{\mathrm{bp}}$.

Pour tout caractère $\mathcal{O}$-arithmétique $\rho$-dominant $\varepsilon \chi$ de $\mathbf{M}_{\Omega}$ pour lequel $U \in \mathcal{T}_{\mathrm{bp}}(\varepsilon)$ et $\chi$ est trivial sur $\mathbf{R}_{\mathbb{R}}^{\mathbb{Q}} \mathbf{G}$, le morphisme

$$
e_{\mathrm{qo}} \mathrm{H}^{d}\left(\mathcal{M}_{U K_{0}}, L_{U}(\rho \otimes \varepsilon \chi, \mathcal{K} / \mathcal{O})\right) \rightarrow e_{\mathrm{qo}} \mathrm{H}^{d}\left(\mathcal{M}_{K_{1}}, L(\mathcal{K} / \mathcal{O})\right)[\varepsilon \chi]
$$

est à noyau et conoyau finis.

Démonstration. - On fixe un plongement complexe $\mathcal{K} \hookrightarrow \mathbb{C}$.

Puisque

$$
\mathrm{H} \cdot\left(\mathcal{M}_{U K_{0}}, L(\rho \otimes \chi, \mathbb{C})\right)=\mathrm{H} \cdot\left(\mathcal{M}_{U K_{0}}, L(\rho \otimes \chi, \mathcal{K})\right) \otimes \mathcal{K} \mathbb{C}
$$

le théorème précédent montre que

$$
\forall i<d, \quad \mathrm{H}^{i}\left(\mathcal{M}_{U K_{0}}, L(\rho \otimes \chi, \mathcal{K})\right)=0 .
$$

De plus, d'après le corollaire 4.3.3 et le lemme 4.3.2, on a

$$
\mathrm{H} \cdot\left(\mathcal{M}_{U K_{0}}, L(\rho \otimes \varepsilon \chi, \mathcal{K})\right)=\mathrm{H}^{0}\left(C_{U}, \mathrm{H} \cdot\left(\mathcal{M}_{U K_{1}}, L(\rho \otimes \chi, \mathcal{K})\right)(\varepsilon)\right) .
$$

Cela donne les annulations nécessaires pour appliquer le théorème 5.4.2.

\subsubsection{Niveau premier à $p$}

Soit $L(\rho, \mathcal{O}):=\operatorname{ind}_{\mathbf{P}_{\Omega}}^{\mathbf{G}_{\Omega}} \rho$ le module de la représentation $\mathcal{O}$-algébrique de $\mathbf{G}_{\Omega}$ induite à partir de $\rho$. En notant $\mathcal{O}\left(\mathbf{G}_{\Omega}\right)$ la $\mathcal{O}$-algèbre des fonctions $\mathcal{O}$-algébriques sur $\mathbf{G}_{\Omega}, L(\rho, \mathcal{O})$ est le $\mathcal{O}$-module formé des fonctions $f \in \mathcal{O}\left(\mathbf{G}_{\Omega}\right) \otimes_{\mathcal{O}} V(\mathcal{O})$ telles que

$$
\forall(g, t, n) \in \mathbf{G}_{\Omega}(\mathcal{O}) \times \mathbf{M}_{\Omega}(\mathcal{O}) \times \mathbf{U}_{\Omega}(\mathcal{O}), \quad f(g t n)=\rho(t)^{-1} f(g)
$$

muni de l'action algébrique donnée par $(g f)(h)=f\left(g^{-1} h\right)$ quels que soient $f \in L(\rho, \mathcal{O})$ et $g, h \in \mathbf{G}_{\Omega}(\mathcal{O})$.

Rappelons que $\mathbf{G}_{\Omega}\left(\mathbb{Z}_{p}\right)$ est supposé spécial.

Selon la décomposition de Cartan [10, I.4.4.3] et la propriété (ii) de [10, I.4.4.4], le sousensemble

$$
D_{p}:=\mathbf{G}_{\Omega}\left(\mathbb{Z}_{p}\right) \sigma\left(\Lambda_{\mathbf{M}}^{+}\right) \mathbf{G}_{\Omega}\left(\mathbb{Z}_{p}\right) \subset \mathbf{G}\left(\mathbb{Q}_{p}\right)
$$

est un sous-monoïde et l'application canonique

$$
\sigma\left(\Lambda_{\mathbf{M}}^{+}\right) \stackrel{\sim}{\rightarrow} \mathbf{G}_{\Omega}\left(\mathbb{Z}_{p}\right) \backslash D_{p} / \mathbf{G}_{\Omega}\left(\mathbb{Z}_{p}\right)
$$

est bijective. L'application réciproque, qui prolonge $\delta$, est encore notée $\delta$.

On étend l'action de $\mathbf{G}_{\Omega}\left(\mathbb{Z}_{p}\right)$ sur le module induit $L(\rho, \mathcal{O})$ pour en faire une action $*$ du sous-monoïde $\left(D_{p}\right)^{-1}: \xi^{-1} * f:=\left(\omega_{\rho} \circ \delta\right)(\xi)\left(\xi^{-1} f\right)$ pour tout $\xi \in D_{p}$ et tout $f \in L(\rho, \mathcal{O})$.

Il faut montrer que l'action ainsi définie stabilise le réseau $L(\rho, \mathcal{O})$. Puisque $\mathbf{R M}_{\mathcal{O}}$ est un tore déployé, la représentation algébrique $L(\rho, \mathcal{O})$ se décompose ( $c f$. [21, II.2.2.5]) aussi sous la forme

$$
L(\rho, \mathcal{O})=\bigoplus_{\chi \in \mathrm{X}^{*}(\mathbf{R M})^{+}} L(\rho, \mathcal{O})\left[\omega_{\rho} \chi\right]
$$


où $\mathbf{R M}\left(\mathbb{Z}_{p}\right)$ agit sur $L(\rho, \mathcal{O})\left[\omega_{\rho} \chi\right]$ par le caractère, à valeurs dans $\mathcal{O}^{\times}$par compacité, induit par $\omega_{\rho} \chi$. Si $f \in L(\rho, \mathcal{O})\left[\omega_{\rho} \chi\right]$, on a donc

$$
t^{-1} * f=\chi(t)^{-1} f \quad \text { pour tout } t \in \sigma\left(\Lambda_{\mathbf{M}}^{+}\right)
$$

et l'inégalité $\omega(\chi(t)) \leqslant 0$ montre la stabilité voulue.

Pour tout $\mathcal{O}$-module $M$, on note $L(\rho, M):=L(\rho, \mathcal{O}) \otimes_{\mathcal{O}} M$.

Pour tout $U \in \mathcal{T}_{\mathrm{bp}}, L(\rho, \mathcal{O})$ est un sous-réseau de $L_{U}(\rho, \mathcal{O})$. D'après 6.1.5, l'action qui vient d'être définie coïncide, sur $\left(U D_{0, p}\right)^{-1}$, avec l'action induite $L_{U}(\rho, \mathcal{O})$.

On considère le sous-groupe de niveau spécial en $p$ suivant :

$$
K:=K^{(p)} \mathbf{G}_{\Omega}\left(\mathbb{Z}_{p}\right) .
$$

On note encore $e_{\mathrm{qo}}$ l'idempotent construit à partir des opérateurs de Hecke $K \xi K, \xi \in \sigma\left(\Lambda_{\mathbf{M}}^{+}\right)$.

Proposition 6.3.1. - Pour tout $U \in \mathcal{T}_{\mathrm{bp}}$, le morphisme canonique

$$
e_{\mathrm{qo}} \mathrm{H}^{\cdot}\left(\mathcal{M}_{K}, L(\rho, \mathcal{K} / \mathcal{O})\right) \stackrel{\sim}{\rightarrow} e_{\mathrm{qo}} \mathrm{H}^{\cdot}\left(\mathcal{M}_{U K_{0}}, L_{U}(\rho, \mathcal{K} / \mathcal{O})\right)
$$

est un isomorphisme.

Démonstration. - On reprend la technique de la démonstration de la proposition 6.2.1 et le morphisme naturel $L(\rho, \mathcal{O}) \rightarrow V(\mathcal{O})$.

Pour adapter cette démonstration au fait que l'espace $\mathbf{G}_{\Omega}\left(\mathbb{Z}_{p}\right)$, contrairement à $U \mathbf{P}_{\Omega}\left(\mathbb{Z}_{p}\right)$, n'est pas dans la grosse cellule, d'après la décomposition de Bruhat, il suffit de tenir compte des éléments du groupe de Weyl. Les arguments de [19, lemme 7.2] et [34, prop. 3.2] s'appliquent encore.

\subsubsection{Annulation de la cohomologie quasi-ordinaire en degré zéro}

Lemme 6.3.1. - On suppose que $\mathbf{P}$ est un sous-groupe parabolique propre de $\mathbf{G}$. Pour tout $U \in \mathcal{T}_{\text {bp }}, e_{\mathrm{qo}} \mathrm{H}_{*}^{0}\left(\mathcal{M}_{U K_{0}}, L_{U}(\rho, \mathcal{K} / \mathcal{O})\right)=0$.

Démonstration. - Puisque $\mathbf{P}$ est propre, son radical unipotent $\mathbf{U}$ n'est pas trivial et il existe $t \in \sigma\left(\Lambda_{\mathbf{M}}^{+}\right)$tel que pour tout caractère $\chi \in \mathrm{X}^{*}(\mathbf{R M})^{+}$non trivial, $\omega(\chi(t))<0$.

Cette inégalité montre que $t^{-1} \in \sigma\left(\Lambda_{\mathbf{M}}^{+}\right)^{-1}$ agit, sur chaque terme de la décomposition

$$
L(\rho, \mathcal{O})=\bigoplus_{\chi \in \mathrm{X}^{*}(\mathbf{R M})^{+}} L(\rho, \mathcal{O})\left[\omega_{\rho} \chi\right]
$$

par un entier de $\mathcal{K}$ de valuation strictement positive, excepté sur le terme correspondant à $\chi=1$.

De plus, $\sigma\left(\Lambda_{\mathrm{M}}^{+}\right)$agit trivialement sur $L(\rho, \mathcal{O})\left[\omega_{\rho}\right]$. Donc l'opérateur de Hecke correspondant à la classe double de $t \in \sigma\left(\Lambda_{\mathrm{M}}^{+}\right)$agit sur les invariants par l'indice $\#(K \backslash K t K)$. La propriété de contraction montre que, pour une puissance de $t$ suffisamment grande, cet indice n'est pas une unité $p$-adique. Ce qui montre la nullité de la partie quasi-ordinaire.

\subsubsection{Problème de congruence et annulation en degré un}

Le problème de congruence pour un groupe semi-simple simplement connexe $\tilde{\mathbf{G}}$ sur $\mathbb{Q}$ consiste en la question suivante :

un sous-groupe arithmétique (i.e. commensurable à $\tilde{\mathbf{G}}(\mathbb{Z})$ ) contient-il nécessairement un sousgroupe de congruence (i.e. le noyau d'un morphisme de réduction $\tilde{\mathbf{G}}(\mathbb{Z}) \rightarrow \tilde{\mathbf{G}}(\mathbb{Z} / m \mathbb{Z}), m \in \mathbb{N}$ ?) 
Bien qu'un plongement $\tilde{\mathbf{G}} \subset \mathbf{G L}(n)$ soit nécessaire pour définir $\tilde{\mathbf{G}}(\mathbb{Z}):=\tilde{\mathbf{G}}(\mathbb{Q}) \cap \mathbf{G L}(n, \mathbb{Z})$, la notion de sous-groupe arithmétique ainsi que le fait de contenir un sous-groupe de congruence ne dépendent pas du choix de ce plongement.

Inversement, il est évident qu'un sous-groupe de congruence est arithmétique.

On note $\overline{\tilde{\mathbf{G}}(\mathbb{Q})}$ le complété de $\tilde{\mathbf{G}}(\mathbb{Q})$ pour la topologie dont un système fondamental de voisinages de l'unité est formé des sous-groupes arithmétiques.

La topologie de $\tilde{\mathbf{G}}(\mathbb{Q})$ dont un système fondamental de voisinages de l'unité est formé des sous-groupes de congruence, est la topologie induite par la topologie du groupe localement profini $\tilde{\mathbf{G}}\left(\mathbb{A}_{\mathrm{f}}\right)$.

$\mathrm{Si} \tilde{\mathbf{G}}$ vérifie le théorème d'approximation forte, le morphisme naturel

$$
\widehat{\tilde{\mathbf{G}}(\mathbb{Q})} \rightarrow \tilde{\mathbf{G}}\left(\mathbb{A}_{\mathrm{f}}\right)
$$

est donc surjectif. Son noyau $C(\tilde{\mathbf{G}})$, appelé noyau de congruence, mesure le défaut du problème de congruence ( $c f$. [2, IV]). Il n'est pas fini en général, par exemple pour $\tilde{\mathbf{G}}=\mathbf{S L}(2)$, c'est un groupe profini libre de rang dénombrable.

Soient $F$ un corps de nombre, dont on note $I_{1}(F)$ l'ensemble des places réelles, et $\mathbf{H}$ un groupe simplement connexe, simple défini sur $F$. Une conjecture de Serre (citée dans [25, (9.45)]) est que

- si $\sum_{v \in I_{1}(F)} \operatorname{rg}_{F_{v}} \mathbf{H} \geqslant 2$ alors $C\left(\mathrm{R}_{F / \mathbb{Q}} \mathbf{H}\right)$ est fini,

- si $\sum_{v \in I_{1}(F)} \operatorname{rg}_{F_{v}} \mathbf{H}=1$ alors $C\left(\mathrm{R}_{F / \mathbb{Q}} \mathbf{H}\right)$ est infini.

Dans sa thèse, K. Buecker $[12, \S 3]$ prouve la nullité de la partie quasi-ordinaire $e_{\mathrm{qo}} \mathrm{H}^{1}\left(\mathcal{M}_{U K_{0}}\right.$, $\left.L_{U}(\rho \otimes \varepsilon \chi)\right)$ de la cohomologie de degré un, dans le cas du groupe symplectique $\mathbf{G}=\mathbf{G S p}(4)$. Sa méthode, qui consiste d'abord à montrer que le noyau d'un cocycle contient un sous-groupe de congruence, et donc se ramener à la cohomologie de groupes finis, se généralise aux groupes dont le noyau de congruence (du revêtement simplement connexe $\tilde{\mathbf{G}}$ du groupe dérivé) est fini d'ordre premier à $p$.

Platonov et Rapinchuk indiquent de nombreux cas pour lesquels $C(\tilde{\mathbf{G}})$ est fini, en dualité avec le noyau métaplectique de $\tilde{\mathbf{G}}$, ce dernier étant plus facilement déterminé ( $c f$. [25, th. 9.15 et 9.23]).

Considérons le groupe $\mathbf{G} \mathbf{U}$ des similitudes unitaires en trois variables d'une extension $\mathrm{CM} E$ d'un corps totalement réel $F$. On suppose qu'en toute place réelle sauf une, GU est anisotrope et qu'en toute place au-dessus de $p, \mathbf{G U}$ est quasi-déployé. Nous nous intéressons à la quasiordinarité relative à un sous-groupe de Borel en $p$.

Le domaine symétrique $X^{\text {ad }}$ de $\mathbf{G} \mathbf{U}^{\text {ad }}$ est une 2-boule complexe (donc $d=2$ ), et les variétés de Shimura de GU sont des surface de Picard (cf. [17]). Quant au domaine symétrique de $\mathbf{G U}$, il est produit de cette 2-boule complexe par $\mathbb{R}^{[F: \mathbb{Q}]-1}$ car le radical de $\mathbf{G U}$ est $\mathbf{G}_{\mathbf{m} E}$, qui est de rang $[F: \mathbb{Q}]$ sur $\mathbb{R}$ et de rang un sur $\mathbb{Q}$. Le tore $\mathbf{R}_{\mathbb{R}}^{\mathbb{Q}} \mathbf{G}$ est ici le noyau de la norme $\mathrm{R}_{F / \mathbb{Q}} \mathbf{G}_{\mathbf{m}} \rightarrow \mathbf{G}_{\mathbf{m}}$.

Si $F=\mathbb{Q}$ alors $\mathbf{G U}$ est de rang semi-simple déployé un sur $\mathbb{Q}$ et la compactification de BorelSerre consiste à élargir $X^{\text {ad }}$ en rajoutant des 2-sphères complexes.

Sinon, $\mathrm{R}_{F / \mathbb{Q}} \mathbf{G U}$ est de rang semi-simple déployé nul sur $\mathbb{Q}$ et les variétés de Shimura de niveau fini $\mathcal{M}_{K}$ sont compactes.

Dans tous les cas, la conjecture de Serre implique que le noyau de congruence du groupe spécial unitaire correspondant à GU est infini.

THÉORÈME 6.3.3. - Soient $U \in \mathcal{T}_{\mathrm{bp}}$ et $\mathbf{G}=\mathrm{R}_{F / \mathbb{Q}} \mathbf{G U}$. 
On suppose que $\rho$ est de plus haut poids régulier, ${ }^{1}$ trivial sur $\mathbf{R}_{\mathbb{R}}^{\mathbb{Q}} \mathbf{G}$ et

$$
\text { p ne divise pas l'ordre de } e_{\mathrm{qo}} \mathrm{H}^{2}\left(\mathcal{M}_{K}, L(\rho, \mathcal{O})\right)_{\text {tor }}
$$

où $K$ est le sous-groupe spécial en $p: K=K^{(p)} \mathbf{G}_{\Omega}\left(\mathbb{Z}_{p}\right)$. Pour tout caractère $\mathcal{O}$-arithmétique $\rho$-dominant $\varepsilon \chi$ de $\mathbf{M}_{\Omega}$ pour lequel $U \in \mathcal{T}_{\mathrm{bp}}(\varepsilon)$ et $\chi$ est trivial sur $\mathbf{R}_{\mathbb{R}}^{\mathbb{Q}} \mathbf{G}$, le morphisme

$$
e_{\mathrm{qo}} \mathrm{H}_{!}^{2}\left(\mathcal{M}_{U K_{0}}, L_{U}(\rho \otimes \varepsilon \chi, \mathcal{K} / \mathcal{O})\right) \stackrel{\sim}{\rightarrow} e_{\mathrm{qo}} \mathrm{H}_{!}^{2}\left(\mathcal{M}_{K_{1}}, L(\mathcal{K} / \mathcal{O})\right)[\varepsilon \chi]
$$

est un isomorphisme.

Démonstration. - L'annulation des groupes de cohomologie quasi-ordinaire (totale) en degré $0(c f .6 .3 .4)$ et 1 (dû à l'hypothèse $\left(\mathrm{TF}_{\rho}\right)$ et à la proposition 6.3.1) permet d'appliquer le théorème de contrôle "fort" 5.4.1:

$$
e_{\mathrm{qo}} \mathrm{H}^{2}\left(\mathcal{M}_{U K_{0}}, L_{U}(\rho \otimes \varepsilon \chi, \mathcal{K} / \mathcal{O})\right) \stackrel{\sim}{\rightarrow} e_{\mathrm{qo}} \mathrm{H}^{2}\left(\mathcal{M}_{K_{1}}, L(\mathcal{K} / \mathcal{O})\right)[\varepsilon \chi] .
$$

Pour un groupe de rang semi-simple 1 sur $\mathbb{Q}$, la suite spectrale de Leray ( $c f$. proposition 4.3.1) du bord devient simplement une décomposition de la cohomologie du bord en une somme directe des cohomologies des composantes du bord.

\subsection{Propriétés de $\mathbf{h}_{\mathrm{qo}}(\rho)$}

À partir de maintenant, $\rho$ est toujours de plus haut poids régulier, trivial sur $\mathbf{R}_{\mathbb{R}}^{\mathbb{Q}} \mathbf{G}$.

On appelle algèbre de Hecke $\mathbf{P}$-quasi-ordinaire universelle l'algèbre

$$
\mathbf{h}_{U, \mathrm{qo}}(\rho):=\mathrm{h}_{!, U, \mathrm{qo}}^{d}\left(K_{1}, \rho\right) .
$$

Par définition ( $c f$. ce qui précède le corollaire 6.2.1), c'est la sous- $\mathcal{O}[[C]]$-algèbre engendrée par les opérateurs de $\mathcal{H}^{(p)}$ agissant sur le module de cohomologie intérieure quasi-ordinaire $e_{\mathrm{qo}} \mathrm{H}_{!}^{d}\left(\mathcal{M}_{K_{1}}, L_{U}(\rho, \mathcal{K} / \mathcal{O})\right)$, ou bien sur son dual de Pontryagin. Ce dernier, noté $\mathbb{V}_{U}(\rho)$, est donc un $\mathbf{h}_{U, \mathrm{qo}}(\rho)$-module compact et fidèle.

\subsubsection{Finitude}

Puisque $\mathcal{K}$ contient les racines $n$-ièmes de l'unité, où $n$ est l'exposant de $C^{(p)}$, on a la décomposition

$$
\begin{aligned}
\mathcal{O}\left[C^{(p)}\right] \stackrel{\sim}{\rightarrow} \prod_{\nu \in \operatorname{Hom}\left(C^{(p)}, \mathcal{O} \times\right)} \mathcal{O}(\nu) \\
c \mapsto(\nu(c))_{\nu} .
\end{aligned}
$$

Proposition 6.4.1. - Soit $U \in \mathcal{T}_{\mathrm{bp}}$.

Le module $\mathbb{V}_{U}(\rho)$ est de type fini sur $\mathcal{O}[[C]]$.

L'algèbre $\mathbf{h}_{U, \mathrm{qo}}(\rho)$ est finie sur $\mathcal{O}[[C]]$.

\footnotetext{
${ }^{1}$ Le rapporteur de l'article nous a signalé le résultat suivant, de D. Blasius et J. Rogawski [3, lemme 4.2.1], qui montre la nécessité de l'hypothèse de régularité dans le théorème précédent : le transfert endoscopique tordu d'une représentation automorphe $\pi$ de $\mathrm{R}_{F / \mathbb{Q}} \mathbf{G L}(2)$ à $\mathrm{R}_{F / \mathbb{Q}} \mathbf{G U}$ ne donne jamais lieu à une seule représentation galoisienne $l$-adique irréductible de dimension 2 dans la cohomologie étale $\mathrm{H}^{2}\left(\mathcal{M}_{U K_{0}}, \mathbb{Z}_{l}\right)$ lorsque $F$ est de degré pair et $\pi$ n'est ramifiée (ou même principale) en aucune place finie. Lorsque $\rho$ est triviale, les théorèmes de contrôle, fort comme faible, sont donc mis en défaut.
} 
Démonstration. - La décomposition précédente donne :

$$
e_{\mathrm{qo}} \mathrm{H}^{d}\left(\mathcal{M}_{K_{1}}, L_{U}(\rho, \mathcal{K} / \mathcal{O})\right)=\bigoplus_{\nu} e_{\mathrm{qo}} \mathrm{H}^{d}\left(\mathcal{M}_{K_{1}}, L_{U}(\rho, \mathcal{K} / \mathcal{O})\right)[\nu]
$$

où $\nu$ parcourt l'ensemble fini des caractères $\nu: C^{(p)} \rightarrow \mathcal{O}^{\times}$.

Soit $\nu$ un tel caractère. On peut construire un caractère fini continu $\varepsilon$ de $\mathbf{M}_{\Omega}\left(\mathbb{Z}_{p}\right)$ qui, passant au quotient $C$, prolonge $\nu$.

Le lemme 4.3.2 donne

$$
e_{\mathrm{qo}} \mathrm{H}^{d}\left(\mathcal{M}_{K_{1}}, L_{U}(\rho, \mathcal{K} / \mathcal{O})\right)(\varepsilon)=e_{\mathrm{qo}} \mathrm{H}^{d}\left(\mathcal{M}_{K_{1}}, L_{U}(\rho, \mathcal{K} / \mathcal{O})(\varepsilon)\right) .
$$

Si on prend les invariants sous $C$, on obtient :

$$
\mathrm{H}^{d}\left(\mathcal{M}_{K_{1}}, L_{U}(\rho, \mathcal{K} / \mathcal{O})\right)[\varepsilon]=\mathrm{H}^{0}\left(C, \mathrm{H}^{d}\left(\mathcal{M}_{K_{1}}, L_{U}(\rho, \mathcal{K} / \mathcal{O})(\varepsilon)\right)\right) .
$$

Selon le théorème 6.3.2, ce dernier groupe est de cotype fini sur $\mathcal{O}$. D'après le lemme de Nakayama topologique, on en déduit que

$$
\mathrm{H}^{d}\left(\mathcal{M}_{K_{1}}, L_{U}(\rho, \mathcal{K} / \mathcal{O})\right)[\nu]=\mathrm{H}^{0}\left(C^{(p)}, \mathrm{H}^{d}\left(\mathcal{M}_{K_{1}}, L_{U}(\rho, \mathcal{K} / \mathcal{O})(\varepsilon)\right)\right)
$$

est de cotype fini sur $\mathcal{O}\left[\left[C_{p}\right]\right]$. Ce qui, d'après la décomposition (22), démontre la première assertion.

La deuxième assertion est une conséquence de la première puisque $\mathbf{h}_{U \text {,qo }}(\rho)$ agit fidèlement $\operatorname{sur} \mathbb{V}_{U}(\rho)$.

\subsubsection{Spécialisation}

Soient $U \in \mathcal{T}_{\mathrm{bp}}, \varepsilon \chi$ un caractère $\mathcal{O}$-arithmétique $\rho$-dominant de $\mathbf{M}_{\Omega}$ tel que $\chi$ est trivial sur $\mathbf{R}_{\mathbb{R}}^{\mathbb{Q}} \mathbf{G}$ et $U^{\prime} \in \mathcal{T}_{\text {bp }}(\varepsilon)$ un sous-groupe de $U$.

On note $\mathrm{h}_{!, U, \mathrm{qo}}\left(U^{\prime} K_{0}, \rho \otimes \varepsilon \chi\right)$ la sous- $\mathcal{O}[[C]]$-algèbre engendrée par l'image de l'anneau de Hecke abstrait $\mathcal{H}^{(p)}$ dans l'algèbre des endomorphismes du $\mathcal{O}[[C]]$-module

$$
e_{\mathrm{qo}} \mathrm{H}_{!}\left(\mathcal{M}_{U^{\prime} K_{0}}, L_{U}(\rho \otimes \varepsilon \chi, \mathcal{K} / \mathcal{O})\right) .
$$

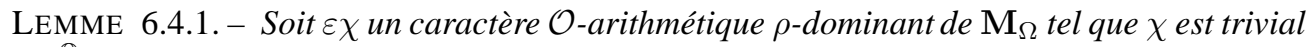
$\operatorname{sur} \mathbf{R}_{\mathbb{R}}^{\mathbb{Q}} \mathbf{G}$.

On a un morphisme naturel entre $\mathcal{K}$-algèbres finies

$$
\mathrm{h}_{U, \mathrm{qo}}^{d}\left(U^{\prime} K_{0}, \rho \otimes \varepsilon \chi\right) \otimes_{\mathcal{O}} \mathcal{K} \nleftarrow \mathbf{h}_{U, \mathrm{qo}}(\rho) \otimes_{\mathcal{O}[[C]]} \mathcal{K}(\varepsilon \chi)
$$

surjectif, à noyau contenu dans le radical.

Démonstration. - En prenant le dual de Pontryagin puis en tensorisant par $\mathcal{K}$, le morphisme du théorème 6.3.2 devient un isomorphisme :

$$
e_{\mathrm{qo}} \mathrm{H}^{d}\left(\mathcal{M}_{U^{\prime} K_{0}}, L_{U}(\rho \otimes \varepsilon \chi, \mathcal{K} / \mathcal{O})\right)^{\vee} \otimes_{\mathcal{O}} \mathcal{K} \stackrel{\sim}{\leftarrow} \mathbb{V}_{U}(\rho) \otimes_{\mathcal{O}[[C]]} \mathcal{K}(\varepsilon \chi)
$$

qui induit un morphisme surjectif sur les algèbres de Hecke :

$$
\mathrm{h}_{U, \mathrm{qo}}^{d}\left(U^{\prime} K_{0}, \rho \otimes \varepsilon \chi\right) \otimes_{\mathcal{O}} \mathcal{K} \nleftarrow \mathbf{h}_{U, \mathrm{qo}}(\rho) \otimes_{\mathcal{O}[[C]]} \mathcal{K}(\varepsilon \chi)
$$


car, par platitude de $\mathcal{K}$ sur $\mathcal{O}$, la première algèbre agit fidèlement sur le premier module de l'isomorphisme précédent.

Pour montrer que ce morphisme surjectif entre $\mathcal{K}$-algèbres finies est à noyau contenu dans le radical, il suffit de montrer que l'image d'un idempotent $\bar{e}$ non nul est non nul.

D'après le lemme d'Hensel cet idempotent se relève en un idempotent $e$ de l'algèbre

$$
\mathbf{h}_{U, \mathrm{qo}}(\rho) \otimes_{\mathcal{O}[[C]]} \mathcal{O}[[C]]_{\mathfrak{p}_{\varepsilon \chi}}
$$

Par platitude de $\mathcal{O}[[C]]_{\mathfrak{p}_{\varepsilon \chi}}$ sur $\mathcal{O}$, cette algèbre de Hecke agit fidèlement sur le module $\mathbb{V}_{U}(\rho)_{\mathfrak{p}_{\varepsilon \chi}}$. Ainsi $e$ est un projecteur de ce module et vérifie

$$
e\left(\mathbb{V}_{U}(\rho)_{\mathfrak{p}_{\varepsilon \chi}}\right) \subset \mathfrak{p}_{\varepsilon \chi} \mathbb{V}_{U}(\rho)_{\mathfrak{p}_{\varepsilon \chi}} .
$$

Ce qui implique $e=0$.

\subsubsection{Algèbre de Hida-Iwasawa}

Soit $C_{0}$ l'adhérence dans $\mathbf{R G}_{\Omega}\left(\mathbb{Z}_{p}\right)$ du sous-groupe $\mathbf{R G}(\mathbb{Q}) \cap \mathbf{R G}_{\Omega}\left(\mathbb{Z}_{p}\right) \subset \mathbf{R G}\left(\mathbb{Q}_{p}\right)$.

Les opérateurs de Hecke construits à partir des éléments de l'intersection $\mathbf{R G}(\mathbb{Q}) \cap \mathbf{R G} \mathbf{G}_{\Omega}\left(\mathbb{Z}_{p}\right)$ agissent trivialement sur la cohomologie de $L_{U}(\rho)$, si bien que l'action de $C$ sur $\mathbb{V}_{U}(\rho)$ se factorise par le groupe $C / C_{0}$.

L'algèbre $\Lambda:=\mathcal{O}[[H]]$, où $H \subset C / C_{0}$ est le sous-groupe pro- $p$ maximal, est l'algèbre de Hida-Iwasawa.

\subsubsection{Liberté}

On note $\chi^{\vee}$ l'image du caractère $\chi$ par l'élément de plus grande longueur $w_{0}$ du groupe de Weyl de $\mathbf{G}$ par rapport à $\mathbf{T}$ qui transforme $\mathbf{B}$ en $\mathbf{B}^{-}$, et on note aussi $\rho^{\vee}$ la représentation de $\mathbf{M}$ de plus grand poids $\chi_{\rho}$ pour l'ordre défini par $\mathbf{B} \cap \mathbf{M}$.

Proposition 6.4.2. - Pour $\mathbf{G}=\mathrm{R}_{F / \mathbb{Q}}$ GU et p satisfaisant aux conditions $\left(\mathrm{TF}_{\rho}\right)$ et $\left(\mathrm{TF}_{\rho} \vee\right)$, le module $\mathbb{V}_{U}(\rho)$ est libre de type fini sur l'algèbre de Hida-Iwasawa $\Lambda$.

Démonstration. - Le lemme 10.2 de [18] montre que l'intersection des ker $\mathfrak{p}_{\chi}$, lorsque $\chi$ parcourt l'ensemble des caractères $\rho$-dominants est nulle. En utilisant le lemme de Nakayama, il suffit donc $(c f .[18, \S 10])$ de montrer que $\mathbb{V}_{U}(\rho \otimes \chi) / \mathfrak{p}_{\chi} \mathbb{V}_{U}(\rho \otimes \chi)$ est libre sur $\mathcal{O}$ pour tout caractère $\mathcal{O}$-algébrique $\rho$-dominant $\chi$.

D'après l'hypothèse $\left(\mathrm{TF}_{\rho^{\vee}}\right), e_{\mathrm{qo}} \mathrm{H}^{2}\left(\mathcal{M}_{K_{0}}, L_{U}\left(\rho^{\vee} \otimes \chi^{\vee}, \mathcal{O}\right)\right)$ n'a pas de $p$-torsion.

Or, 2 étant la dimension médiane de $\mathcal{M}$, ce dernier est en dualité ( $c f .[34$, th. 6.4] ou [1]) avec

$$
e_{\mathrm{qo}} \mathrm{H}_{c}^{2}\left(\mathcal{M}_{K_{0}}, L_{U}(\rho \otimes \chi, \mathcal{K} / \mathcal{O})\right)
$$

qui est donc $p$-divisible.

Il en est de même de la cohomologie intérieure, qui est image de la cohomologie à support compact. L'isomorphisme du théorème de contrôle 6.3.3 donne donc

$$
e_{\mathrm{qo}} H_{!}^{2}\left(\mathcal{M}_{K_{1}}, L_{U}(\rho, \mathcal{K} / \mathcal{O})\right)\left[\mathfrak{p}_{\chi}\right] \stackrel{\sim}{\rightarrow} e_{\mathrm{qo}} H_{!}^{2}\left(\mathcal{M}_{K_{0}}, L_{U}(\rho \otimes \chi, \mathcal{K} / \mathcal{O})\right) .
$$

En passant au dual, on obtient bien que $\mathbb{V}_{U}(\rho \otimes \chi) / \mathfrak{p}_{\chi} \mathbb{V}_{U}(\rho \otimes \chi)$ est libre sur $\mathcal{O}$.

\subsubsection{Dimension}

Nous notons $\operatorname{rgp} \mathbf{P}$ le rang parabolique (absolu) de $\mathbf{P}$, c'est-à-dire le cardinal de l'ensemble des sous-groupes propres maximaux contenant $\mathbf{P}$. 
Proposition 6.4.3. - La dimension de Krull relative de l'algèbre de Hida-Iwasawa sur $\mathcal{O}$ est

$$
\begin{aligned}
\operatorname{rg}_{\mathbb{Z}_{p}} H & =\operatorname{rgp} \mathbf{P}+\operatorname{dim} \mathbf{R G}-\operatorname{rg}_{\mathbb{Z}} \mathbf{R G}(\mathbb{Z})+\delta_{\mathbf{R G}, p} \\
& =\operatorname{rgp} \mathbf{P}+\operatorname{dim} \mathbf{R G}+\operatorname{rg}_{\mathbb{Q}} \mathbf{R G}-\operatorname{rg}_{\mathbb{R}} \mathbf{R G}+\delta_{\mathbf{R G}, p}
\end{aligned}
$$

où

$$
\delta_{\mathbf{R G}, p}:=\operatorname{rg}_{\mathbb{Z}} \mathbf{R G}(\mathbb{Z})-\operatorname{rg}_{\mathbb{Z}_{p}} \overline{\mathbf{R G}(\mathbb{Z})}^{p}
$$

et $\overline{\mathbf{R G}(\mathbb{Z})}^{p}$ est l'adhérence de $\mathbf{R G}(\mathbb{Z})$ dans $\mathbf{R G}\left(\mathbb{Z}_{p}\right)$.

Lorsque $\mathbf{R G}$ est un tore $\mathbf{R}_{E / \mathbb{Q}} \mathbf{G}_{\mathbf{m}}$ obtenu par restriction des scalaires alors $\delta_{\mathbf{R G}, p}$ est le défaut $\delta_{E, p}$ de la conjecture de Leopoldt pour le corps $E$ en $p$. La conjecture de Leopoldt a été démontrée par A. Brumer [11] dans le cas des extensions abéliennes $E / \mathbb{Q}$.

Démonstration. - La première égalité résulte du fait que la dimension du tore $\mathbf{R M}$ est la somme de la dimension du radical $\mathbf{R G}$ et du rang parabolique de $\mathbf{P}$ :

$$
\operatorname{rg}_{\mathbb{Z}_{p}} \mathbf{R M}\left(\mathbb{Z}_{p}\right)=\operatorname{dim} \mathbf{R G}+\operatorname{rgp} \mathbf{P}
$$

La seconde vient de la définition de $\delta_{\mathbf{R G}, p}$ et du théorème de Dirichlet pour les tores [25, cor. 1 du th. 4.14]: $\operatorname{rg}_{\mathbb{Z}} \mathbf{R G}(\mathbb{Z})=\operatorname{rg}_{\mathbb{R}} \mathbf{R G}-\operatorname{rg}_{\mathbb{Q}} \mathbf{R G}$.

COROllaire 6.4.1. - Dans la même situation que la proposition 6.4.2, l'algèbre de Hecke quasi-ordinaire universelle $\mathbf{h}_{U, \mathrm{qo}}(\rho)$ est finie et sans torsion sur l'algèbre de Hida-Iwasawa $\Lambda$. Elle est de dimension relative

$$
\operatorname{rg}_{\mathbb{Z}_{p}} H=1+3[F: \mathbb{Q}]+\delta_{E, p}
$$

$\operatorname{sur} \mathcal{O}$.

Démonstration. - Ce sont les conséquences des deux propositions précédentes car le radical de $\mathbf{G}=\mathrm{R}_{F / \mathbb{Q}} \mathbf{G} \mathbf{U}$ est $\mathbf{R G}=\mathrm{R}_{E / \mathbb{Q}} \mathbf{G}_{\mathbf{m}}$ et l'on a :

$$
\operatorname{rgp} \mathbf{P}=2[F: \mathbb{Q}], \quad \operatorname{dim} \mathbf{R G}=2[F: \mathbb{Q}], \quad \operatorname{rg}_{\mathbb{Q}} \mathbf{R G}=1,
$$

et

$$
\operatorname{rg}_{\mathbb{R}} \mathbf{R G}=[F: \mathbb{Q}], \quad \delta_{\mathbf{R G}, p}=\delta_{E, p} .
$$

\subsubsection{Systèmes de valeurs propres}

Si II est une $\Lambda$-algèbre finie et sans torsion, on dit qu'un idéal premier de II est arithmétique s'il est au-dessus d'un idéal de la forme $\mathfrak{p}_{\varepsilon \chi}$, où $\varepsilon \chi$ est un caractère $\mathcal{O}$-arithmétique de $\mathbf{M}_{\Omega}$. Et un caractère $\mathbb{I} \rightarrow \mathcal{O}$ est arithmétique s'il prolonge un caractère arithmétique de $\Lambda$.

Une famille de systèmes de valeurs propres quasi-ordinaires est un morphisme $\mathbf{h}_{U \text {,qo }}(\rho) \rightarrow \mathbb{I}$ de $\Lambda$-algèbres où $\mathbb{I}$ est une $\Lambda$-algèbre finie sans torsion.

Une remarque importante est que, puisque $\mathbb{I}$ est un anneau local, deux caractères arithmétiques sont congrus modulo l'uniformisante de $\mathcal{O}$. Cette propriété et la proposition suivante permettent de "construire" des congruences.

La manière dont nous avons modifié l'action originale de $\mathbf{G}(\mathcal{K})$ sur $L(\rho, \mathcal{K})$ nous amène à donner la définition suivante : un système de valeurs propres $\Theta$ intervenant en plus haut poids cohomologique $\lambda$ (pour l'ordre associé à $\mathbf{B}^{-}$comme au paragraphe 6.1.1) est $\mathbf{P}$-quasi-ordinaire 
si et seulement si $\left|\Theta\left(T_{p}(\xi)\right)\right|_{p}=|\lambda(\xi)|_{p}$ pour tout $\xi \in \sigma\left(\Lambda_{\mathbf{M}}^{+}\right)$, où $T_{p}(\xi)$ est l'opérateur de Hecke construit à partir de $\xi$ (pour l'action originale).

Proposition 6.4.4. - Soit $\Theta_{\pi}$ un système de valeurs propres pour les opérateurs de Hecke, associé à une représentation automorphe cuspidale de $\mathbf{G}=\mathrm{R}_{F / \mathbb{Q}} \mathbf{G} \mathbf{U}$, dont la composante archimédienne est de poids cohomologique régulier.

Alors pour presque tout $p$, si $\Theta_{\pi}$ est quasi-ordinaire, il existe une famille de systèmes de valeurs propres quasi-ordinaires passant par $\Theta_{\pi}$.

Démonstration. - En effet, par hypothèse, à $\pi$ est associée une représentation irréductible $V_{\lambda}$ de $\mathbf{G}$ de plus haut poids régulier $\lambda$ (pour l'ordre défini par $\mathbf{B}^{-}$). Cette représentation peut être construite sous la forme $L(\rho, \mathbb{C})$ en prenant pour $\rho$ l'induite de $\lambda$ de $\mathbf{B} \cap \mathbf{M}$ à $\mathbf{M}$.

On peut choisir $\mathcal{K}$ suffisamment gros pour qu'il contienne les valeurs propres et d'après la proposition 6.3.1, on peut se placer en niveau $U K_{0}$ (i.e. de type $\Gamma_{0}$ ) en $p$, pour $\Omega$ réduit à un point spécial bien placé par rapport à $(\mathbf{P}, \mathbf{M})$ et un sous-groupe $U \in \mathcal{T}_{\text {bp }}$.

Puisque le conoyau du morphisme

$$
\mathrm{H}_{!}^{2}\left(\mathcal{M}_{U K_{0}}, L_{U}(\rho \otimes \varepsilon \chi, \mathcal{K})\right) \rightarrow \mathrm{H}_{!}^{2}\left(\mathcal{M}_{U K_{0}}, L_{U}(\rho \otimes \varepsilon \chi, \mathcal{K} / \mathcal{O})\right)
$$

est fini, le système de valeurs propres intervient aussi pour les coefficients divisibles $L_{U}(\rho \otimes$ $\varepsilon \chi, \mathcal{K} / \mathcal{O})$.

Si bien que $\Theta_{\pi}$ est un morphisme

$$
\mathrm{h}_{!, U, \mathrm{qo}}^{2}\left(U K_{0}, \rho \otimes \varepsilon \chi\right) \stackrel{\Theta_{\pi}}{\rightarrow} \mathcal{O}
$$

qui, composé avec l'application $\mathbf{h}_{U, \mathrm{qo}}(\rho) \rightarrow \mathrm{h}_{!, U, \mathrm{qo}}^{2}\left(U K_{0}, \rho \otimes \varepsilon \chi\right)$ donne un morphisme dont le noyau $\mathfrak{P}$ est au-dessus de l'idéal arithmétique $\mathfrak{p}_{\varepsilon \chi}$. Le "going-down" $[9, \mathrm{~V}, \S 2$, th. 3] donne un idéal premier $\mathfrak{p} \subset \mathbf{h}_{U \text {,qo }}(\rho)$ contenu dans $\mathfrak{P}$, au-dessus de $\{0\} \subset \Lambda$.

Posons $\mathbb{I}:=\mathbf{h}_{U, \mathrm{qo}}(\rho) / \mathfrak{p}$. C'est une $\Lambda$-algèbre finie qui se décompose en un produit d'anneaux locaux car $\Lambda$ est complète. Or $\mathbb{I}$ est intègre par construction, donc $\mathbb{I}$ est un anneau local, sans torsion sur $\Lambda$.

Ainsi, on a le diagramme commutatif suivant:

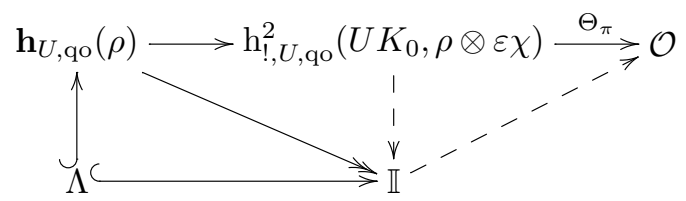

où le morphisme de $\mathbf{h}_{U, \mathrm{qo}}(\rho)$ se factorise $\operatorname{par} \mathrm{h}_{!, U, \mathrm{qo}}^{d}\left(U K_{0}, \rho \otimes \varepsilon \chi\right)$ d'après le lemme 6.4.1 et le morphisme de $\mathbb{I}$ dans $\mathcal{O}$ est obtenu par réduction modulo $\mathfrak{P} / \mathfrak{p}$.

Ce morphisme indique que la famille construite passe par $\Theta_{\pi}$.

\section{RÉFÉRENCES}

[1] Ash A., Stevens G., $p$-adic deformations of cohomology classes of subgroups of $\mathrm{GL}(n, \mathbf{Z})$, Collectanea Math. 48 (1-2) (1997) 1-30.

[2] Bass H., Milnor J., SERre J.-P., Solution of the congruence subgroup problem for $\mathrm{SL}_{n}(n \geqslant 3)$ and $\mathrm{Sp}_{2 n}(n \geqslant 2)$, Publ. Math. IHÉS 33 (1967) 59-137. 
[3] Blasius D., Rogawski J.D., Motives for Hilbert modular forms, Invent. Math. 114 (1) (1993) 5587.

[4] BOREL A., Introduction aux groupes arithmétiques, Hermann, 1969.

[5] Borel A., Serre J.-P., Corners and arithmetic groups, Comment. Math. Helv. 48 (1974) 436-491.

[6] Borel A., Tits J., Groupes réductifs, Publ. Math. IHÉS 27 (1965) 55-151, avec compléments dans 41 (1972) 253-276.

[7] BourbaKi N., Groupes et algèbres de Lie, Masson, 1971-1998.

[8] BourbaKi N., Topologie générale, Masson, 1974-1990.

[9] BOURBAKI N., Algèbre commutative, Masson, 1983-1998.

[10] Bruhat F., Tits J., Groupes réductifs sur un corps local I II et III, Publ. Math. IHÉS 4160 (1972) 5-252, 5-184, 1984, J. Fac. Sci. Univ. Tokyo 34 (1987) 671-698.

[11] BRUMER A., On the units of algebraic number fields, Mathematika 14 (1967) 121-124.

[12] Buecker K., On the control theorem for the symplectic group, Compositio Math. 113 (1) (1998) 91-115.

[13] DeLIGne P., Variétés de Shimura : Interprétation modulaire, et techniques de construction de modèles canoniques, in: A. Borel, W. Casselman (Eds.), Automorphic Forms, Representations, and Lfunctions, in: Proc. Symp. in Pure Math., vol. 33, 1979, pp. 247-289.

[14] Demazure M., Gabriel P., Groupes Algébriques - Tome I, Masson/North-Holland, 1970.

[15] Demazure M., Grothendieck A., Schémas en groupes, in : Lecture Notes in Math., vol. 151, 152, 153, Springer-Verlag, 1962-1964.

[16] Godement R., Théorie des faisceaux, Hermann, 1958.

[17] Gordon B.B., Canonical models of Picard modular surfaces, in : R.P. Langlands, D. Ramakrishnan (Eds.), The $\zeta$-functions of Picard Modular Surfaces, in: Proceedings, vol. 13, Les Publications CRM, 1992, pp. 1-29.

[18] HIDA H., On $p$-adic Hecke algebras for $G L(2)$ over totally real fields, Ann. of Math. 128 (1988) 295-384.

[19] HIDA H., Control theorems of $p$-nearly ordinary cohomology groups for $S L(n)$, Bull. Soc. Math. France 123 (1995) 425-475.

[20] HIDA H., Automorphic induction and Leopoldt type conjectures for GL(n), Asian J. Math. 2 (4) (1998) 667-710, Mikio Sato: a great Japanese mathematician of the twentieth century.

[21] JAnTZEn J.C., Representations of Algebraic Groups, in : Pure and Applied Math., vol. 131, Academic Press, 1987.

[22] Kuga M., PARry W., SAH C.H., Group cohomology and Hecke operators, in: Manifolds and Lie Groups (Notre Dame, Ind., 1980), Birkhäuser, 1981, pp. 223-266.

[23] J.-S. LI, J. SChWERMER. On the Eisenstein cohomology of arithmetic groups, Duke Math. J., à paraître.

[24] MiYaKe T., Modular Forms, Springer-Verlag, 1989.

[25] Platonov V., Rapinchuk A., Algebraic Groups and Number Theory, in : Pure and Applied Math., vol. 139, Academic Press, 1994.

[26] Raynaud M., Anneaux locaux henséliens, in: Lecture Notes in Math., vol. 169, Springer-Verlag, 1970.

[27] S APER L.D., $\mathcal{L}$-modules and micro-support, Prépublication disponible sur math.RT/0112251, 2001.

[28] SAPER L.D., $\mathcal{L}$-modules and the conjecture of Rapoport and Goresky-MacPherson, in: Tilouine, Carayol, Harris, Vigneras (Eds.), Formes automorphes, Actes du Semestre du Centre É. Borel, IHP, Paris février-juillet 2000, 2001, Astérisque. Soc. Math. France, à paraître. Disponible sur math.RT/0112250.

[29] SATAKE I., Theory of spherical functions on reductive algebraic groups over $p$-adic fields, Publ. Math. IHÉS 18 (1963) 5-69.

[30] Schneider P., StUhler U., Representation theory and sheaves on the Bruhat-Tits building, Publ. Math. IHÉS 85 (1997) 97-191.

[31] SerRe J.-P., Corps locaux, in: Publ. Math. de l'Univ. de Nancago, vol. 8, Hermann, 1968.

[32] SERRE J.-P., Cohomologie galoisienne, in : Lecture Notes in Math., vol. 5, Springer-Verlag, 1994.

[33] Shimura G., Introduction to the Arithmetic Theory of Automorphic Functions, Princeton Univ. Press, 1971. 
[34] Tilouine J., Urban É., Several-variable $p$-adic families of Siegel-Hilbert cusp eigensystems and their Galois representations, Ann. Sci. Éc. Norm. Sup. 32 (1999) 499-574.

[35] Tits J., Buildings of Spherical Type and Finite BN-Pairs, in: Lecture Notes in Math., vol. 386, Springer-Verlag, 1974.

(Manuscrit reçu le 3 janvier 2002; accepté, après révision, le 13 janvier 2004.)

\author{
David MAUGER \\ Institut de Mathématiques de Jussieu, \\ Université Paris 7, \\ Case postale 7012, \\ 2 place Jussieu, \\ F-75251 Paris cedex 05, France \\ E-mail :mauger@math.jussieu.fr
}

